\title{
ON LOCATION AND APPROXIMATION OF CLUSTERS OF ZEROES: CASE OF EMBEDDING DIMENSION ONE
}

\author{
M. GIUSTI, G. LECERF, B. SALVY, AND J.-C. YAKOUBSOHN
}

\begin{abstract}
Isolated multiple zeroes or clusters of zeroes of analytic maps with several variables are known to be difficult to locate and approximate. This article is in the vein of the $\alpha$-theory, initiated by M. Shub and S. Smale in the beginning of the eighties. This theory restricts to simple zeroes, i.e., where the map has corank zero. In this article we deal with situations where the analytic map has corank one at the multiple isolated zero, which has embedding dimension one in the frame of deformation theory. These situations are the least degenerate ones and therefore most likely to be of practical significance. More generally, we define clusters of embedding dimension one. We provide a criterion for locating such clusters of zeroes and a fast algorithm for approximating them, with quadratic convergence. In case of a cluster with positive diameter our algorithm stops at a distance of the cluster which is about its diameter.
\end{abstract}

\section{Contents}

Introduction

1. $\alpha$-Theory for Simple Zeroes $\quad 9$

2. Local Compositional Inverse 17

3. Reduction to One Variable 21

4. Cluster Location 27

5. Cluster Approximation 30

6. Numerical Experiments 40

$\begin{array}{ll}\text { References } & 46\end{array}$

Date: Preliminary version of October 25, 2004.

2000 Mathematics Subject Classification. Primary 65H10; Secondary 32-04, 32S99.

Key words and phrases. $\alpha$-theory, cluster approximation, cluster location, cluster of zeroes, Newton's operator, Pellet's criterion, Rouché's theorem, Schröder's operator, zeroes of analytic maps. 


\section{INTRODUCTION}

In both theory and practice, dealing with multiple zeroes and clusters of zeroes of multivariate analytic maps remains a challenging problem. The now classical $\alpha$-theory developed by M. Shub and S. Smale restricts to simple zeroes. At such zeroes the map is of minimal corank, i.e., zero in our context, and this property remains of course valid in an open neighborhood. In this article we treat the next case, i.e., where the corank of the analytic map is 1 at the multiple zero. Note that this implies, through the implicit function theorem, that the zero lies on a smooth locus of a curve, hence its embedding dimension is 1 . In the context of numerical analysis, the right issue is to isolate and approximate clusters of zeroes, simple or not. Therefore, the right hypothesis is not the corank at most 1 condition in a ball, but the embedding 1 condition, as we exemplify in the next paragraphs. Our generalization of the $\alpha$-theory is done under this latter hypothesis, which actually covers a large class of singularies encountered in practice.

Preliminaries. We start by setting basic definition and conventions used all along this text. Then, we introduce and illustrate the embedding dimension 1 condition.

First Definitions and Conventions. We denote by $\mathbb{R}$ the field of real numbers, by $\mathbb{C}$ the field of complex numbers and by $\imath \in \mathbb{C}$ the square root of -1 with positive imaginary part. For any $z \in \mathbb{R}$ (resp. $z \in \mathbb{C}),|z|$ denotes the absolute value of $z$ (resp. the modulus of $z$ ). On $\mathbb{C}^{n}$ we consider the canonical Hermitian norm denoted $\|$.$\| . Let \zeta \in \mathbb{C}^{n}$ and $r \geq 0$ be a real number, then we let $B(\zeta, r):=\left\{x \in \mathbb{C}^{n},\|x-\zeta\|<r\right\}$ and $\bar{B}(\zeta, r):=\left\{x \in \mathbb{C}^{n},\|x-\zeta\| \leq r\right\}$. For any real number $u$ and any integer $m \geq 1$, we introduce the auxiliary function

$$
\psi_{m}(u):=2(1-u)^{m+1}-1 .
$$

We will also use $\psi(u):=\psi_{1}(u)=1-4 u+2 u^{2}$. For a compact subset $Z$ of $\mathbb{C}^{n}$, the diameter of $Z$ is the maximum distance between any two points of $Z$.

Let $f$ denote an analytic map from an open subset $U$ of $\mathbb{C}^{n}$ to $\mathbb{C}^{m}$ and $a \in U$. Then its $k$ th derivative $D^{k} f(a)$ belongs to the space $\mathcal{L}_{k}\left(\mathbb{C}^{n} ; \mathbb{C}^{m}\right)$ of $\mathbb{C}$-multilinear maps from $k$ copies of $\mathbb{C}^{n}$ to $\mathbb{C}^{m}$. For any $L \in \mathcal{L}_{k}\left(\mathbb{C}^{n} ; \mathbb{C}^{m}\right)$, we use the following norm:

$$
\|L\|:=\sup _{\substack{u_{1} \in \mathbb{C}^{n}, \ldots, u_{k} \in \mathbb{C}^{n} \\\left\|u_{1}\right\|=\cdots=\left\|u_{k}\right\|=1}}\left\|L\left(u_{1}, \ldots, u_{k}\right)\right\| .
$$

If $h$ denotes a one variable complex function then $h^{(k)}$ (resp. $h^{\prime}$ and $\left.h^{\prime \prime}\right)$ represents its $k$ th derivative (resp. the first and the second derivative). We denote by $\mathbb{R}\{t\}$ the algebra of real power series with positive radius of convergence. We say that a series $\lambda t /(1-\rho t)$ is a geometric series, for the sequence of its coefficients is in geometric progression. We widely use the following notation for the exponential generating series of the norms of the 
derivatives of $f$ at $a \in \mathbb{C}^{n}$ :

$$
[f]_{a}:=\sum_{k \geq 0}\left\|D^{k} f(a)\right\| \frac{t^{k}}{k !} \in \mathbb{R}\{t\} .
$$

We consider the following partial order $\leq$ over $\mathbb{R}\{t\}$. Let $F$ and $G$ be in $\mathbb{R}\{t\}$, we write $F \leq G$ when $F^{(k)}(0) \leq G^{(k)}(0)$ for all $k \geq 0$. Then we say that a power series $F \in \mathbb{R}\{t\}$ is a majorant series for an analytic map $f$ at a point $a$ if $[f]_{a} \leq F$. For the sake of completeness, Section 1 provides the main properties on majorant series we use in this article.

Concerning Newton's operator, we will use the following notation:

$$
N(f ; x):=x-D f(x)^{-1} f(x),
$$

and for Schröder's operator [43] (if $f$ is a univariate map) we write:

$$
N_{m}(f ; x):=x-m \frac{f(x)}{f^{\prime}(x)} .
$$

Of course, for analytic functions, $N_{1}$ and $N$ coincide.

Point Estimates. So called point estimates are quantities defined from norms of differential maps at a given point. Let $f$ denote an analytic map from $U \subseteq \mathbb{C}^{n}$ to $\mathbb{C}^{n}$ and $a$ a point of $U$. Three important such quantities are used for simple zeroes: $\gamma, \beta$ and $\alpha$. The first one, namely $\gamma(f ; a)$, helps control the function locally:

$$
\gamma(f ; a):=\sup _{k \geq 2}\left\|D f(a)^{-1} \frac{f^{(k)}(a)}{k !}\right\|^{\frac{1}{k-1}} .
$$

In particular, the radius of convergence of the power series expansion of $f$ at $a$ is at least $1 / \gamma$. The second quantity is the length of the Newton iteration step: $\beta(f ; a):=\left\|D f(a)^{-1} f(a)\right\|$; the third one is their product $\alpha(f ; a):=\beta(f ; a) \gamma(f ; a)$. In terms of geometric majorant series, it will be convenient to use

$$
F(f, a ; t):=\beta(f ; a)+\frac{t}{1-\gamma(f ; a) t}, \text { since }\left[D f(a)^{-1} f\right]_{a} \leq F(f, a ; t) .
$$

For univariate maps, in order to deal with clusters of $m$ zeroes, counting multiplicities, and multiple zeroes of multiplicity $m$, the previous quantities are generalized as follows, for any $l \in\{0, \ldots, m-1\}$ :

$$
\begin{aligned}
\gamma_{m}(f ; a) & :=\sup _{k \geq m+1}\left(\frac{m !\left|f^{(k)}(a)\right|}{k !\left|f^{(m)}(a)\right|}\right)^{\frac{1}{k-m}}, \\
\beta_{m, l}(f ; a) & :=\sup _{l \leq k \leq m-1}\left(\frac{m !\left|f^{(k)}(a)\right|}{k !\left|f^{(m)}(a)\right|}\right)^{\frac{1}{m-k}}, \\
\alpha_{m, l}(f ; a) & :=\gamma_{m}(f ; a) \beta_{m, l}(f ; a) .
\end{aligned}
$$

As expected, these quantities coincide with the previous ones when letting $m=1$ and $l=0$. For short we let $\beta_{m}:=\beta_{m, 0}$ and $\alpha_{m}:=\alpha_{m, 0}$. If $\gamma_{m}=0$ then we consider $1 / \gamma_{m}=+\infty$, as a natural convention. 
Clusters of Embedding Dimension One. Formally speaking, by cluster we only mean set. In the context of clusters of zeroes, this word is convenient to refer to a set of zeroes whose diameter is small compared to the distance to other zeroes.

Let $F$ be an analytic map defined on a connected open subset $U \subseteq \mathbb{C}^{n}$ to $\mathbb{C}^{n}$. A set of zeroes of $F$ is said to be a cluster of zeroes of embedding dimension 1 if there exist two vector subspaces $S$ and $T$ of $\mathbb{C}^{n}$ of codimension 1 and a ball $B(\zeta, r) \subseteq U$ containing the cluster and such that $\operatorname{Pr}_{T} \circ D F(x)$ is invertible on $S$, for all $x \in B(\zeta, r)$, where $\operatorname{Pr}_{T}$ denotes the orthogonal projection to $T$.

In this situation, one can choose orthonormal bases at the source and the target of $F$ that satisfy: if $(x, y)=\left(x_{1}, \ldots, x_{n-1}, y\right)$ are the new coordinates at the source and $\left(f_{1}, \ldots, f_{n-1}, g\right)$ at the target then the partial derivative of $f=\left(f_{1}, \ldots, f_{n-1}\right)$ with respect to the variables $x$ is invertible in $B(\zeta, r)$. Let $\zeta_{x}$ and $\zeta_{y}$ respectively denote the $x$ and $y$ coordinates of $\zeta$. The implicit function theorem implies that there exists an analytic function $\phi(y): W \rightarrow \mathbb{C}^{n-1}$ defined on a neighborhood $W$ of $\zeta_{y}$ such that $\phi\left(\zeta_{y}\right)=\zeta_{x}$ and $f(\phi(y), y)=0$ holds in $W$. Up to restricting $W, h(y):=g(\phi(y), y)$ is well defined on $W$. Looking for zeroes of $F$ is then reduced to looking for zeroes on a smooth curve, and, after parametrization, for zeroes of a univariate analytic function. This motivated us to use this embedding dimension terminology.

Note that multiple zeroes of corank 1 are clusters of embedding dimension 1 . In addition, this embedding dimension 1 property is preserved under small deformations (by deformation, we refer to the classical theory given in [3]).

Example 1: The common archetype is the map

$$
\left(x_{1}, \ldots, x_{n-1}, y\right) \mapsto\left(x_{1}, \ldots, x_{n-1}, y^{m}\right),
$$

which admits the origin as a zero of multiplicity $m$ and corank 1 . All multiple zeroes of corank 1 and multiplicity $m$ are analytically equivalent to this example.

Example 2: Fat point of multiplicity 4 and corank 1. Let us consider an ellipse and one of its superosculating circle. They intersect at such a point: coordinates can be easily found when the situation is described by the equations $x_{1}^{2}-4 x_{1}+4 y^{2}$ and $x_{1}^{2}-x_{1}+y^{2}$ (here $n=2$ ).

Counterexample: Let $F:=\left(x_{1}, y\right) \mapsto\left(x_{1}^{2}-e, y^{2}-e\right)$, for a positive real parameter $e$ and consider the cluster composed of the four zeroes of $F$. Since the origin belongs the convex hull of this cluster, any ball containing the cluster also contains the origin. It follows that this cluster can not have embedding dimension 1 since $D F(0,0)=0$.

All along this text, we assume that the map $F$ is already given with well suited sets of coordinates $(x, y)$ and $(f, g)$ that satisfy the above properties. If this were not the case it would suffice to perform a random change of orthonormal coordinates. The methods presented here for cluster location and approximation are not designed to treat all clusters of embedding dimension 1 but only the ones that are sufficiently small in some sense we precisely quantify in terms of point estimates. 
Main Notation. The following notation is used in the remainder of the introduction and, then, from Section 3.3: in the first sections, we present fundamental results that we need but which are not specific to clusters of embedding dimension 1.

Let $U \subseteq \mathbb{C}^{n}$ be an open subset, $\zeta \in U, x$ the set of the $n-1$ first coordinates and $y$ the last coordinate of $\mathbb{C}^{n} ; \zeta_{x}$ and $\zeta_{y}$ represent the coordinates in $x$ and $y$ respectively of $\zeta$. Let $f: U \rightarrow \mathbb{C}^{n-1}$ and $g: U \rightarrow \mathbb{C}$ be two analytic maps. When $U$ is connected, the open set $U_{f}$ and $U_{g}$ respectively denote the maximal analyticity domains of $f$ and $g$ that contain $U$. Instead of considering only the implicit function defined when $f$ equals 0 , we introduce the following map $\Sigma$ that allows one to handle the family of implicit functions defined when $f$ equals a certain parameter that varies in a neighborhood of 0 . Note that this map is classical for proving the equivalence between the local compositional inverse function and the implicit function theorems. Namely, for any $\left(x_{0}, y_{0}\right) \in U$ such that $D_{x} f\left(x_{0}, y_{0}\right)$ is invertible, we introduce

$$
\begin{aligned}
& \Sigma\left(f, x_{0}, y_{0} ; x, y\right): \quad U \rightarrow \mathbb{C}^{n} \\
& (x, y) \mapsto\left(D_{x} f\left(x_{0}, y_{0}\right)^{-1} f(x, y), y\right),
\end{aligned}
$$

which is invertible in a neighborhood of $\left(x_{0}, y_{0}\right)$, its inverse is denoted by $\Phi\left(f, x_{0}, y_{0} ; z, y\right)$ and is defined on a neighborhood of $\left(z_{0}, y_{0}\right)$, where $z_{0}:=$ $D_{x} f\left(x_{0}, y_{0}\right)^{-1} f\left(x_{0}, y_{0}\right)$. We also introduce

$$
h\left(f, g, x_{0}, y_{0} ; z, y\right):=g\left(\Phi\left(f, x_{0}, y_{0} ; z, y\right)\right) .
$$

For convenience, we also use the notation $\phi\left(f, x_{0}, y_{0} ; y\right)$ for representing the $n-1$ first coordinates of $\Phi\left(f, x_{0}, y_{0} ; 0, y\right)$, so

$$
\left(\phi\left(f, x_{0}, y_{0} ; y\right), y\right)=\Phi\left(f, x_{0}, y_{0} ; 0, y\right)
$$

holds. Then, in a certain neighborhood of $\left(x_{0}, y_{0}\right)$ (that will be described precisely when needed) the system $f(x, y)=g(x, y)=0$ becomes equivalent to

$$
h\left(f, g, x_{0}, y_{0} ; 0, y\right)=0, \quad x=\phi\left(f, x_{0}, y_{0} ; y\right),
$$

hence reduces to considering a univariate equation.

By construction, we have $f\left(\phi\left(f, x_{0}, y_{0} ; y\right), y\right)=0$, hence $\phi$ does not depend on $\left(x_{0}, y_{0}\right)$ in a neighborhood of $\left(x_{0}, y_{0}\right)$. In particular, $\phi$ actually represents the parameterization of the implicit function defined by $f=0$. Recall that if $\left(x_{0}, y_{0}\right)$ is an isolated solution of $f=g=0$ then its multiplicity equals the multiplicity of $y_{0}$ as a solution of $h\left(f, g, x_{0}, y_{0} ; 0, y\right)=0$.

For any $l \geq 0$, all along this text, we study the $l$ th deflated map $\left(f, g^{[l]}\right)$ obtained from $(f, g)$ according to the following recursive definition:

$$
g^{[0]}=g, \quad g^{[l+1]}=\frac{\operatorname{det}\left(D\left(f, g^{[l]}\right)\right)}{\operatorname{det}\left(D_{x} f\right)}, \quad \text { for } l \geq 0,
$$

where det denotes the determinant map, the implicit basis that we consider is the canonical one given by $(x, y)$. Lastly, when writing $h\left(f, g, x_{0}, y_{0} ; z,.\right)$, we mean the single complex variable map of $y$, for fixed $z$. 
Our Contributions. In this article we provide a numerical criterion for locating clusters of embedding dimension 1 . Then we exhibit and analyze a natural approximation algorithm and show that quadratic approximation is possible with an overhead cost that is only polynomial in the multiplicity: roughly speaking, the main feature of our algorithm is that it stops the iteration at a distance of the cluster which is proved to be about the diameter of this cluster. Our techniques naturally extend and rely on the results of [12] for location and approximation of clusters of zeroes of analytic functions in one variable.

The first section is devoted to prerequisites on majorant series and classical results on location and approximation of simple zeroes, following the now classical $\alpha$-theory. We provide a new synthetic shorter presentation of Pellet's location criterion, the $\gamma$ - and $\alpha$-theorems, thanks to the mechanical majorant series point of view. In the vein of Kantorovich's analysis, we generalize the $\alpha$-theorem given in [55] in order to show that if Pellet's criterion is satisfied at $x_{0}$ then the Newton iterates of $x_{0}$ converge to a simple zero.

In the frame of our main notation above, given $\left(x_{0}, y_{0}\right) \in U$, for short we write $h(z, y)$ instead of $h\left(f, g, x_{0}, y_{0} ; z, y\right)$. All along this text we often make use of such abbreviations. From a theoretical point of view, the multivariate situation on which we focus here reduces to one variable when considering the equation $h(0,)=$.0 , as explained above. In order to turn this basic idea into practical algorithms two main ingredients are needed: first a quantitative version of the implicit function theorem and then a quantification of the variations of point estimates of $h(z, y)$ when $z$ varies for fixed $y$.

Implicit functions are handled via local inversion, which is treated in Section 2. We improve the recent result given in [8]: namely, we provide a sharper geometric series majoration of the inverse map, in cases where the derivative at the given point is different from identity.

Section 3 is devoted to quantify how $\beta_{m, l}(h(z,) ; y$.$) and \gamma_{m}(h(z,) ; y$.$) vary$ with respect to $z$ when $y$ is constant. The common archetype the reader may keep in mind is $h(z, y)=z y^{l}+y^{m}$ : the diameter of the cluster of zeroes of $D_{y}^{l} h(z,)=$.0 varies like $\|z\|^{1 /(m-l)}$. Therefore in order to approximate $D_{y}^{l} h(0, y)$ at a usable scale, $\|z\|^{1 /(m-l)}$ will be required to be sufficiently small. The rest of this section contains a proof of the following relation:

$$
D_{y}^{l} h\left(f, g, x_{0}, y_{0} ; z, y\right)=g^{[l]} \circ \Phi\left(f, x_{0}, y_{0} ; z, y\right),
$$

which provides an efficient way of computing Taylor expansions of $h$. For the sake of completeness, we recall the location criterion of [12] for univariate maps, that we use in next sections. We also recall the relation between diameters of clusters and $\beta_{m, l}$ : briefly speaking, for a univariate function $q$, if $\alpha_{m, l}(q ; a)$ is sufficiently small then $q^{(l)}$ admits a cluster of $m-l$ zeroes in a ball centered at $a$ of radius about $\beta_{m, l}(q ; a)$. In addition, if $a$ lies in the convex hull of this cluster then the diameter of the cluster is also about $\beta_{m, l}(q ; a)$. This crucial result allows one to get approximations of diameters of clusters. When we say that a point lies far from or close to the cluster, we refer to its diameter as the implicit scale. 
Section 4 deals with cluster location. This means finding (a) a point; (b) an estimate for the radius of a ball centered at this point and containing zeroes; (c) the radius of a zero-free region beyond this ball. We generalize [12, Section 1], that restricts to univariate functions and [9], that deals with our present case of study but restricting to clusters containing 2 zeroes (that is $m=2$ in our context).

For any $l^{\prime} \leq l$, in Section 5, we generalize the one variable approximation algorithm presented in [12, Section 4]. Roughly speaking, our algorithm proceeds this way: we first compute $\left(x_{0}^{\prime}, y_{0}\right)$ such that $z_{0}^{\prime}:=$ $D_{x} f\left(x_{0}, y_{0}\right)^{-1} f\left(x_{0}^{\prime}, y_{0}\right)$ is of order $2\left(m-l^{\prime}\right)$, then we compute the Schröder iterate $y_{0}^{\prime}:=N_{m-l}\left(D_{y}^{l} h\left(z_{0}^{\prime},.\right) ; y_{0}\right)$. Depending on the position of $y_{0}$ with respect to the cluster of zeroes of $D_{y}^{l^{\prime}} h\left(z_{0}^{\prime},.\right), y_{1}$ will be chosen among $y_{0}$ and $y_{0}^{\prime}$. We also exhibit a stopping criterion ensuring that the whole process stops close to the cluster of $D_{y}^{l^{\prime}} h(0,$.$) .$

When using our algorithm with $l=l^{\prime}=0$, the computation of $y_{0}^{\prime}$ only requires the first derivative of $h$. On the opposite, with $l=m-1$ and $l^{\prime}=0$, our algorithm is close to performing Newton's iteration on the full deflated map. Our stopping criterion then allows one to stop this iteration close to the cluster of the original system. These extreme cases motivate this unified presentation in terms of $l$ and $l^{\prime}$.

The last section presents experimental results and some implementation issues.

Related Work. In the following paragraphs, we discuss other approaches to cluster detection and to generalizations of Newton's iteration. Recall that the univariate case that we generalize here is treated in [12], to which we refer for the bibliography. Here, we focus on several variables maps.

$\alpha$-Theory. In dimension larger than one and in the spirit of the $\alpha$-theory developed by Shub and Smale [46, 44, 45, 47, 4], the only quantitative result generalizing simple zeroes is due to Dedieu and Shub. In [9], they present a qualitative version of Rouché's theorem that guarantees the existence of clusters of two zeroes. In this article, we present a more general criterion that deals with higher multiplicities. We also provide an approximation algorithm based on Schröder's operator [43, 53]. As for simple zeroes, all our results are effective and certified, as exemplified in the last section.

In the next paragraphs, we present other works concerning approximation of multiple zeroes, that are outside the scope of the $\alpha$-theory. This presentation is not intended to be exhaustive but tends to reflect some mainstreams among a vast amount of literature, to which our results are most related.

Deflation. In the multivariate case, the idea of deflation can be generalized to deal with multiple zeroes: deflation algorithms mainly consist in differentiating well chosen equations, according to the nature of the singularity. Ojika, Watanabe, Mitsui [36] and then Ojika [35] proposed a so-called modified deflation algorithm based on hybrid symbolic and numerical computations. In [32], this technique is improved and the number of deflation stages is proved to be bounded by the multiplicity. The questions of complexity and stability of these mixed approaches have not been studied yet. On the other 
hand, in the computer algebra framework (that concerns non archimedian valuated fields) a general deflation algorithm has been described in [31]: this gives an algorithm for quadratic approximation of multiple zeroes in time which remains polynomial in the multiplicity. Yet this method has not led to numerical experiments.

The present work deals with a particular case of deflated systems of depth one, according to the terminology of [31]. The main difference is that we deal with clusters instead of multiple zeroes, which turns out to be much more difficult. Dealing with clusters of embedding dimension higher that 1 by means of deflated systems of depth higher than one is a difficult challenge.

Corrected Newton Methods. Experimentally, if Newton's method converges to an isolated singularity then the convergence is linear. A quantitative analysis of this property is complicated. Several authors have contributed to this topic. Motivated by Ostrowski [37], Rall studied some particular cases in [40]. Then, based on Reddien's advances [41, 42], Decker and Kelley [6, 7] precise the convergence rate for singular problems of first and second orders for maps between Banach spaces. Griewank and Osborne [14, 16, 15] propose generalizations and precise convergence domains.

In the univariate case, corrected operators are well known from Schröder's work [43] and have been improved by Van de Vel [53, 54]. Recently, this approach has been generalized to Pham systems [26].

In general, correcting Newton's iteration to restablish quadratic convergence has been studied in numerous works. In this vein, an important class of approximation methods is based on a so called regularization of the Newton correction: we refer to [2, Theorem 1] for recent advances and references on this subject. In fact, the construction described therein is obtained from the one we mention in the next paragraph.

Bordering Techniques. So-called bordering techniques form a large class of methods to deal with singularities. As for the deflation techniques, the main idea is the construction of a system that admits a simple solution in place of the singular solution of the original system, hence Newton's operator can be used. For double zeroes, this technique is explored in [56, 57, 15, 22, 23, 33]. Tsuchiya [52] is based on [57] and treats multiple zeroes having corank 1.

Augmented Systems. The construction of augmented systems can also be used to approximate isolated singular zeroes. Roughly speaking, the basic idea consists in introducing new variables corresponding to coordinates of vectors belonging to some kernels of well chosen linear maps constructed from derivatives of the given map and depending on the type of the singularity.

The number of articles dealing with these techniques is too huge to cite them all, we refer to $[17,39,18,13]$ for historical details and references. The most general method is exposed in [30] (as a generalization of [28, 29]) and is extended to Banach spaces in [20].

Cluster Approximation. Among all these techniques, the cluster approximation problem remains unexplored: relating the zeroes of bordered, augmented or deflated systems to the ones of the original problem is still an 
open question in full generality. For the first time, we solve this problem here for clusters of embedding dimension 1. Our use of the implicit function theorem by means of truncated power series computations is a major difference compared to the other aforementioned methods. In particular, we do not introduce extra unknowns at the opposite of the construction of augmented systems.

Algebraic Topology. Far from our present concerns, a vast amount of results for locating zeroes of analytic maps are designed from numerical integration and residue formula. We refer to [27] for an exposition of some of these results and for an historical presentation. Other original zero counting related methods are based on topological degree theory [51, 25].

Global Techniques. Lastly, from a global point of view, it is worth mentioning that several techniques for polynomial system solving are robust in presence of multiplicities and clusters, but their complexities take into account all the zeroes of the systems. Originating from commutative algebra, let us mention recent advances in Gröbner basis computation [10, 11] and geometric solving [31]. From a numerical point of view, formal computations can be used as a preprocess: for instance geometric solving brings back to solving a univariate polynomial, or suitable constructions bring back to linear algebra, as in [5]. Pure numerical techniques mostly rely on homotopy continuation, as in $[34,50,48,49]$, for example.

\section{1. $\alpha$-Theory for Simple Zeroes}

For the sake of completeness this first section gathers materials of $\alpha$-theory for simple zeroes. We start with Pellet's location criterion, which we directly prove via Rouché's theorem. Then we recall the $\gamma$-theorem, generalize [55] to majorant series and prove the $\alpha$-theorem. In this section, we never use our main notation from the introduction.

1.1. Majorant Series. We briefly recall the main basic properties of majorant series and geometric majorant series. We refer to [12, Appendix A] for proofs. Recall that $\mathbb{R}\{t\}$ denotes the algebra of real power series of positive radius of convergence, partially ordered by $\leq$, as defined earlier. This partial order satisfies the following basic compatibility rules, that will be used without explicit reference in the sequel.

Proposition 1.1. The partial order on $\mathbb{R}\{t\}$ satisfies the following compatibility rules:

a. For all nonnegative $x$ in $\mathbb{R}, x \geq 0$, seen as the constant function in $\mathbb{R}\{t\}$;

b. For all $F$ in $\mathbb{R}\{t\}, F \geq 0$ is equivalent to $-F \leq 0$;

c. For all $F, G$ and $H$ in $\mathbb{R}\{t\}$, if $F \leq G$ then $F+H \leq G+H$;

d. For all $F, G$ and $H$ in $\mathbb{R}\{t\}$, if $F \leq G$ and $H \geq 0$ then $F H \leq G H$.

Let $f$ denote an analytic map from a connected open subset $U \subseteq \mathbb{C}^{n}$ to $\mathbb{C}^{m}$. As defined in (1), the map [.] satisfies the following basic properties:

Proposition 1.2. According to the above notation, for any $a \in U$ we have:

a. $[f]_{a} \geq 0$; 
b. $[f]_{a}=0$ is equivalent to $f=0$ in a neighborhood of $a$;

c. $[c f]_{a}=|c|[f]_{a}$, for all $c \in \mathbb{C}$;

d. $[f+g]_{a} \leq[f]_{a}+[g]_{a}$.

Next follows a list of less basic properties. Let $a \in U, F$ be such that $[f]_{a} \leq F$.

Proposition 1.3. (Differentiation) $[D f]_{a} \leq F^{\prime}$.

Let $b \in U$ be such that $\|b-a\|$ is less than the radius of convergence of $F$.

Proposition 1.4. (Evaluation) $\|f(b)\| \leq F(\|a-b\|)$.

Proposition 1.5. (Translation) $[f]_{b} \leq F(t+\|a-b\|)$.

Corollary 1.6. (Translation for geometric series) If $F=F(0)+\frac{\lambda t}{1-\rho t}$, then

$$
[f-f(b)]_{b} \leq \frac{\lambda^{\prime} t}{1-\rho^{\prime} t}
$$

with $\lambda^{\prime}:=\lambda /(1-\rho\|b-a\|)^{2}$ and $\rho^{\prime}:=\rho /(1-\rho\|b-a\|)$.

Proposition 1.7. (Product) Let $f$ be an analytic map from $U$ to $\mathcal{L}\left(\mathbb{C}^{n} ; \mathbb{C}^{m}\right)$ and $g$ be an analytic map from $U$ to $\mathcal{L}\left(\mathbb{C}^{p} ; \mathbb{C}^{n}\right)$, then we have:

$$
[h]_{a} \leq[f]_{a}[g]_{a}
$$

where $h$ is defined by

$$
\begin{aligned}
h: U & \rightarrow \mathcal{L}\left(\mathbb{C}^{p} ; \mathbb{C}^{m}\right) \\
x & \mapsto f(x) \circ g(x) .
\end{aligned}
$$

In particular, it follows that the product of majorant series of univariate functions is a majorant series for the product of these functions.

Proposition 1.8. (Composition) Let $g$ be an analytic map from $\mathbb{C}^{m}$ to $\mathbb{C}^{p}$, defined in the neighborhood of $f(a)$ and $G$ be such that $[g]_{f(a)} \leq G$. Let $h:=g \circ f$, then we have

$$
[h]_{a} \leq G \circ(F-F(0)) .
$$

Corollary 1.9. (Composition for geometric series) According to the notation of the previous proposition, if $F=F(0)+\frac{\lambda_{f} t}{1-\rho_{f} t}, G=G(0)+\frac{\lambda_{g} t}{1-\rho_{g} t}$, then

$$
[h-h(a)]_{a} \leq \frac{\lambda t}{1-\rho t}, \text { where } \lambda:=\lambda_{f} \lambda_{g}, \rho:=\rho_{f}+\lambda_{f} \rho_{g} .
$$

In addition, $f(B(a, 1 / \rho)) \subseteq B\left(f(a), 1 / \rho_{g}\right)$.

Lastly, we recall a formula concerning inversion of linear maps.

Proposition 1.10. (Inversion) Let $U$ be an open neighborhood of a in $\mathbb{C}^{n}$ and $f$ be analytic from $U$ to $\mathcal{L}\left(\mathbb{C}^{m} ; \mathbb{C}^{m}\right)$ such that $f(a)=\mathrm{Id}$. Let $F \in \mathbb{R}\{t\}$ such that $[f]_{a} \leq F$, then

$$
\left[f^{-1}\right]_{a} \leq \frac{1}{1+F(0)-F}
$$

In addition, the radius of convergence of $\frac{1}{1+F(0)-F(t)}$ is at least

$$
\bar{\rho}:=\sup (s<\rho \mid 1+F(0)-F(r)>0, \text { for all } r \in[0, s]),
$$

where $\rho$ denotes the radius of convergence of $F$. 
Proof. The series majoration corresponds to [12, Proposition A.8]. For any $z \in B(0, \bar{\rho})$, the triangular inequality and $F \geq 0$ imply

$$
|1+F(0)-F(z)| \geq 1-|F(z)-F(0)| \geq 1-F(|z|)+F(0)>0,
$$

which means that $z \mapsto 1+F(0)-F(z)$ does not vanish in $B(0, \bar{\rho})$.

1.2. Location of Simple Zeroes. We show that the optimal location criterion (in terms of the quantity $\alpha$ ) given in [55] can be deduced from Rouché's theorem. We start with the following proposition, that generalizes Pellet's criterion [38] to simple zeroes of several variables maps. We follow the same presentation as in [12, Section 1].

For the rest of this section, $f$ denotes an analytic map from a connected open subset $U \subseteq \mathbb{C}^{n}$ to $\mathbb{C}^{n}, x_{0}$ belongs to $U$ and $D f\left(x_{0}\right)$ is assumed to be invertible. For short we let $\alpha:=\alpha\left(f ; x_{0}\right), \beta:=\beta\left(f ; x_{0}\right), \gamma:=\gamma\left(f ; x_{0}\right)$. For a majorant series $F$, we will often use the notation

$$
\tilde{F}:=F-\left(1+F^{\prime}(0)\right) t .
$$

Proposition 1.11. Let $F \in \mathbb{R}\{t\}$ be such that $\left[D f\left(x_{0}\right)^{-1} f\right]_{x_{0}} \leq F$. Let $r>0$ be a real number smaller than the radius of convergence of $F$ such that $\bar{B}\left(x_{0}, r\right) \subseteq U$ and

$$
\tilde{F}(r)<0
$$

then $f$ has exactly one simple zero in $\bar{B}\left(x_{0}, r\right)$.

Proof. First observe that it is not restrictive to assume $D f\left(x_{0}\right)=\mathrm{Id}$. We introduce $g: U \rightarrow \mathbb{C}^{n}, g(x):=f(x)-f\left(x_{0}\right)$. Let $w \neq x_{0}$ be such that $s:=$ $\left\|w-x_{0}\right\| \leq r$. By Taylor expansion, we have $g(w)=w-x_{0}+\mathcal{O}_{x_{0}}\left(w-x_{0}\right)^{2}$, then by the triangular inequality, we get

$$
\frac{\|g(w)\|}{s} \geq 1-\sum_{j \geq 2} \frac{F^{(j)}(0)}{j !} s^{j-1} \geq 1-\frac{F(r)-F(0)-r F^{\prime}(0)}{r}>\frac{F(0)}{r},
$$

where the last inequality follows from (5). Here $\mathcal{O}_{x_{0}}$ represents the classical Landau "big O" notation in the neighborhood of $x_{0}$. As a consequence, $x_{0}$ is the only simple zero of $g$ in the ball $\bar{B}\left(x_{0}, r\right)$. Moreover, when $s=r$, the inequality above yields $\|f(w)-g(w)\|<\|g(w)\|$. In particular, $g(w)$ does not vanish and therefore the multivariate generalization of Rouché's theorem [1, Chapter 1, Theorem 2.5] asserts that $f$ and $g$ have the same number of zeroes in $\bar{B}\left(x_{0}, r\right)$, counting multiplicities.

We now examine the important special case where $F=F\left(f, x_{0} ; t\right)$, as defined in (2): inequality (5) rewrites

$$
\tilde{F}\left(f, x_{0} ; r\right):=\beta-r+\frac{\gamma r^{2}}{1-\gamma r}<0 .
$$

Assuming $\gamma r<1$ and $\bar{B}\left(x_{0}, r\right) \subseteq U$, the previous proposition specializes to: if $\tilde{F}\left(f, x_{0} ; r\right)<0$ then $f$ has exactly one simple zero in $\bar{B}\left(x_{0}, r\right)$.

$\tilde{F}\left(f, x_{0} ; r\right)$ is convex on the range $0 \leq r<1 / \gamma$, and inequality (6) admits solutions in this range if and only if $\alpha<3-2 \sqrt{2}$. In addition, by convexity, this set of solutions forms a range that we write $\left(r^{-}\left(f ; x_{0}\right), r^{+}\left(f ; x_{0}\right)\right)$, where $r^{-}\left(f ; x_{0}\right)$ and $r^{+}\left(f ; x_{0}\right)$ (that may be infinity) are the roots of $\tilde{F}\left(f, x_{0} ; r\right)=0$. 
For short, we let $r^{-}:=r^{-}\left(f ; x_{0}\right)$ and $r^{+}:=r^{+}\left(f ; x_{0}\right)$ and a direct calculation yields:

$$
r^{-}:=\beta \frac{2}{1+\alpha+\sqrt{1-6 \alpha+\alpha^{2}}}, \quad r^{+}:=\frac{1+\alpha+\sqrt{1-6 \alpha+\alpha^{2}}}{4 \gamma} .
$$

If $\alpha \neq 0$ then the following inequality chain follows from the facts that $r^{-}$ is convex increasing while $r^{+}$is concave decreasing as functions of $\alpha$ :

$$
\begin{gathered}
\beta<(1+\alpha) \beta<r^{-}<\left(1+\left(2+3 \frac{\sqrt{2}}{2}\right) \alpha\right) \beta<\left(1+\frac{\sqrt{2}}{2}\right) \beta< \\
\frac{1-\frac{\sqrt{2}}{2}}{\gamma}<\frac{1-(1+\sqrt{2}) \alpha}{2 \gamma}<r^{+}<\frac{1-\alpha}{2 \gamma}<\frac{1}{2 \gamma} .
\end{gathered}
$$

We summarize this discussion in the following theorem.

Theorem 1.12. [55, Proposition 2] If $\alpha<3-2 \sqrt{2}$ then for any $r$ such that $r^{-} \leq r<r^{+}$and $\bar{B}\left(x_{0}, r\right) \subseteq U, f$ has exactly one simple zero in $\bar{B}\left(x_{0}, r\right)$.

It is worth mentioning that this result is sharp, for it applies to $\tilde{F}\left(f, x_{0} ; r\right)$, seen as a univariate map of $r$ : at $r=0$, it is easy to see $\beta\left(\tilde{F}\left(f, x_{0} ;.\right) ; 0\right)=\beta$ and $\gamma\left(\tilde{F}\left(f, x_{0} ;.\right) ; 0\right)=\gamma$. In practice, the next corollary will reveal easier to use:

Corollary 1.13. If $\alpha<3-2 \sqrt{2}$ and $\bar{B}\left(x_{0},(1+\sqrt{2} / 2) \beta\right) \subseteq U$ then $f$ has exactly one simple zero in both $\bar{B}\left(x_{0},(1+\sqrt{2} / 2) \beta\right)$ and $B\left(x_{0},(1-\sqrt{2} / 2) / \gamma\right) \cap$ $U$.

Proof. We apply the previous theorem with the analytic extension of $f$ on $B\left(x_{0}, 1 / \gamma\right)$, for $r=(1+\sqrt{2} / 2) \beta$ (according to (8)) and then for any $r$ in a left neighborhood of $(1-\sqrt{2} / 2) / \gamma$ (according to (9)).

1.3. Translations of Point Estimates. From estimates at a given point, getting upper bounds on the same quantities at another close point is a central operation. The following proposition will be used in proofs of the $\gamma$ and $\alpha$-theorems below.

Proposition 1.14. Let $F$ be such that $\left[D f\left(x_{0}\right)^{-1} f\right]_{x_{0}} \leq F$, then

$$
\left[D f\left(x_{0}\right)^{-1} D f\right]_{x_{0}} \leq F^{\prime} \text { and }\left[D f^{-1} D f\left(x_{0}\right)\right]_{x_{0}} \leq-\frac{1}{\tilde{F}^{\prime}} .
$$

Proof. Again we can assume $D f\left(x_{0}\right)=$ Id and start with differentiating $[f]_{x_{0}} \leq F$ : according to Proposition 1.3 , we obtain $[D f]_{x_{0}} \leq F^{\prime}$. Then the second majoration follows from Proposition 1.10.

Translations of $\alpha, \beta$ and $\gamma$ are useful for various tasks, we shall use them several times.

Proposition 1.15. [4, Chapter 8, Proposition 3] Let $x_{1} \in U, r:=\left\|x_{1}-x_{0}\right\|$ such that $u:=\gamma r<1-\sqrt{2} / 2$, then
a. $\alpha\left(f ; x_{1}\right) \leq \frac{\alpha(1-u)+u}{\psi(u)^{2}}$;
b. $\beta\left(f ; x_{1}\right) \leq \frac{(1-u)}{\psi(u)}(\beta(1-u)+r)$; 
c. $\gamma\left(f ; x_{1}\right) \leq \frac{\gamma}{(1-u) \psi(u)}$;

d. $\left\|D f\left(x_{1}\right)^{-1} D f\left(x_{0}\right)\right\| \leq \frac{(1-u)^{2}}{\psi(u)}$;

e. $\left\|D f\left(x_{0}\right)^{-1} D f\left(x_{1}\right)\right\| \leq \frac{1}{(1-u)^{2}}$.

Proof. Using the previous proposition with $F=F\left(f, x_{0} ; t\right)$, we find:

$$
\left[D f\left(x_{0}\right)^{-1} D f\right]_{x_{0}} \leq \frac{1}{(1-\gamma t)^{2}} \text { and }\left[D f^{-1} D f\left(x_{0}\right)\right]_{x_{0}} \leq \frac{(1-\gamma t)^{2}}{\psi(\gamma t)} .
$$

Parts (d) and (e) directly follow via Proposition 1.4.

Then, we use the translation property for majorant series: according to Proposition 1.5, we have $\left[D f\left(x_{0}\right)^{-1} f\right]_{x_{1}} \leq F\left(f, x_{0} ; r+t\right)$, hence

$$
\begin{aligned}
{\left[D f\left(x_{0}\right)^{-1} f\right]_{x_{1}} } & \leq \beta+\frac{r+t}{1-\gamma(r+t)} \\
& =\beta+\frac{r}{1-u}+\frac{1}{(1-u)^{2}} \frac{t}{1-\frac{\gamma}{1-u} t} .
\end{aligned}
$$

From $\left[D f\left(x_{1}\right)^{-1} f\right]_{x_{1}} \leq\left\|D f\left(x_{1}\right)^{-1} D f\left(x_{0}\right)\right\|\left[D f\left(x_{0}\right)^{-1} f\right]_{x_{1}}$, we deduce:

$$
\left[D f\left(x_{1}\right)^{-1} f\right]_{x_{1}} \leq \frac{(1-u)^{2}}{\psi(u)}\left(\beta+\frac{r}{1-u}+\frac{1}{(1-u)^{2}} \frac{t}{1-\frac{\gamma}{1-u} t}\right),
$$

from which directly follow Parts (b) and (c), and finally (a).

1.4. $\gamma$-Theorem. The $\gamma$-theorem quantifies the convergence of Newton's operator from point estimates at the zero. It is useful for homotopy continuation, when combined to the previous upper bounds on translation.

Theorem 1.16. [4, Chapter 8, Proposition 1] Let $\zeta \in U$ and $r>0$ be a real number such that $f(\zeta)=0, D f(\zeta)$ is invertible, $\bar{B}(\zeta, r) \subseteq U, u:=$ $\gamma(f ; \zeta) r<1-\sqrt{2} / 2$ and $u / \psi(u) \leq 1$. Then, for any $x_{0} \in \bar{B}(\zeta, r)$ the sequence $\left(x_{k}\right)_{k \in \mathbb{N}}$ recursively defined by $x_{k+1}:=N\left(f ; x_{k}\right)$ is well defined, has all elements belonging to $\bar{B}(\zeta, r)$ and

$$
\left\|x_{k}-\zeta\right\| \leq\left(\frac{u}{\psi(u)}\right)^{2^{k}-1}\left\|x_{0}-\zeta\right\|, \text { for all } k \geq 0 .
$$

Proof. A direct calculation gives:

$$
\left[D f(\zeta)^{-1}(D f(x) x-f(x))\right]_{\zeta} \leq t F^{\prime}(f, \zeta ; t)-F(f, \zeta ; t) .
$$

Using Propositions 1.14 and 1.7 then yields:

$$
\begin{aligned}
{\left[x-D f(x)^{-1} f(x)\right]_{\zeta} } & =\left[\left(D f(x)^{-1} D f(\zeta)\right)\left(D f(\zeta)^{-1}(D f(x) x-f(x))\right)\right]_{\zeta} \\
& \leq-\frac{t F^{\prime}(f, \zeta ; t)-F(f, \zeta ; t)}{\tilde{F}(f, \zeta ; t)}=\frac{\gamma(f ; \zeta) t^{2}}{\psi(\gamma(f ; \zeta) t)} .
\end{aligned}
$$

By means of Proposition 1.4, evaluating this series majoration at $x=x_{0}$ and $t=r$ gives

$$
\left\|x_{1}-\zeta\right\| \leq \frac{\gamma(f ; \zeta)}{\psi(u)}\left\|x_{0}-\zeta\right\|^{2} \leq \frac{u}{\psi(u)}\left\|x_{0}-\zeta\right\| \leq\left\|x_{0}-\zeta\right\| .
$$


A straightforward induction concludes the proof.

Combining the previous results actually leads to a weak $\alpha$-theorem (see next subsection). More precisely, let $x_{0} \in U$ be such that $D f\left(x_{0}\right)$ is invertible and $\bar{B}\left(x_{0},(1+\sqrt{2} / 2) \beta\right) \subseteq U$. Let $v:=(1+\sqrt{2} / 2) \alpha$ and assume $v<1-\sqrt{2} / 2$, which is equivalent to $\alpha<3-2 \sqrt{2}$. Then, from Corollary 1.13, there exists a simple zero $\zeta$ in $\bar{B}\left(x_{0},(1+\sqrt{2} / 2) \beta\right)$. Let $u:=\frac{v}{(1-v) \psi(v)}$ and assume $u<1-\sqrt{2} / 2$ and $\frac{u}{\psi(u)} \leq 1$, Proposition 1.15 gives:

$$
\gamma(f ; \zeta) \leq \frac{\gamma}{(1-v) \psi(v)}
$$

hence $\gamma(f ; \zeta)(1+\sqrt{2} / 2) \beta \leq u$ and Theorem 1.16 asserts quadratic convergence from $x_{0}$. The supremum value $\hat{\alpha}$ of the $\alpha$ 's satisfying these conditions is:

$$
\hat{\alpha}:=\sup \left(\frac{v}{1+\sqrt{2} / 2} \mid 0 \leq v<1-\sqrt{2} / 2, u<1-\sqrt{2} / 2, \frac{u}{\psi(u)} \leq 1\right) .
$$

An easy calculation produces $0.06571<\hat{\alpha}<0.06572$. This is not the best possible value. Indeed the optimal condition on $\alpha$ yielding quadratic convergence of Newton iterator is given in [55, Theorem 1]: the critical value is $3-2 \sqrt{2} \approx 0.17157$. This is the next result we recall.

1.5. $\alpha$-Theorem. The $\alpha$-theorem below presents a huge interest in practice since it combines location and quantitive approximation from estimates at the inital point. As in [55], we use the dominating sequence technique, that consists in exhibiting an increasing sequence $t_{k}$ of nonnegative real numbers such that $\left\|x_{k+1}-x_{k}\right\| \leq t_{k+1}-t_{k}$, where $x_{k}$ are the Newton iterates of $x_{0}$. This idea goes back to Kantorovich [24]. The next theorem shows that once Pellet's criterion is satisfied at $x_{0}$ then the Newton iterates of $x_{0}$ converge quadratically to a simple zero. We generalize the original idea of [55] to majorant series. We do not use Proposition 1.11, but provide another proof that does not rely on Rouché's theorem.

Theorem 1.17. Let $F \in \mathbb{R}\{t\}$ be such that $\left[D f\left(x_{0}\right)^{-1} f\right]_{x_{0}} \leq F$. Let $r>0$ be a real number smaller than the radius of convergence of $F$ such that $\bar{B}\left(x_{0}, r\right) \subseteq U$ and $\tilde{F}(r)<0$. Then $f$ has exactly one simple zero $\zeta$ in $\bar{B}\left(x_{0}, r\right)$. In addition, the sequences $\left(x_{k}\right)_{k \in \mathbb{N}}$ recursively defined by $x_{k+1}:=$ $N\left(f ; x_{k}\right)$ and $\left(t_{k}\right)_{k \in \mathbb{N}}$ recursively defined by $t_{0}=0$ and $t_{k+1}:=N\left(\tilde{F} ; t_{k}\right)$ are well defined: $x_{k}$ belongs to $\bar{B}\left(x_{0}, r\right)$, for all $k \geq 0$, converges quadratically to $\zeta, t_{k}$ is increasing and converges quadratically to the first nonnegative root $r^{-}$of $\tilde{F}$. Convergences compare this way:
a. $\left\|x_{k}-x_{k+1}\right\| \leq t_{k+1}-t_{k}$
b. $\left\|D f\left(x_{0}\right)^{-1} f\left(x_{k}\right)\right\| \leq t_{k+1}-t_{k}$;
c. $\left\|x_{k}-\zeta\right\| \leq r^{-}-t_{k}$.

Proof. Let us first deal with the degenerate case when $F^{\prime \prime}=0$. In this situation $x_{k}=\zeta, t_{k}=r^{-}=\beta$, for all $k \geq 1$ and the theorem trivially holds.

From now on, we assume $F^{\prime \prime} \neq 0$. In particular this implies that $\tilde{F}$ is strictly convex on $[0, r]$. Since $\tilde{F}(0) \geq 0$ and $\tilde{F}(r)<0$ we deduce that there exists a unique zero $r^{-}$of $\tilde{F}$ in $[0, r]$. 
From convexity, we also deduce that $t_{k}$ is increasing and converges to $r^{-}$. The dominating sequence technique consists in proving

$$
\left\|x_{k+1}-x_{k}\right\| \leq t_{k+1}-t_{k} .
$$

For this purpose, we proceed by induction on $k$ : if $k=0$ then $\left\|x_{1}-x_{0}\right\|=$ $F(0)=\tilde{F}(0)=t_{1}$, since $\tilde{F}^{\prime}(0)=-1$. Now, we assume that inequality (10) holds up to a certain index $k-1 \geq 0$. First, it is easy to check

$$
\begin{aligned}
\left\|x_{k}-x_{0}\right\| & \leq\left\|x_{k}-x_{k-1}\right\|+\cdots+\left\|x_{1}-x_{0}\right\| \\
& \leq t_{k}-t_{k-1}+\cdots+t_{1}-t_{0}=t_{k}-t_{0} \leq r^{-} .
\end{aligned}
$$

Since $\tilde{F}^{\prime}$ does not vanish on $\left[0, r^{-}\right]$, Proposition 1.10 implies that the radius of convergence of $-1 / \tilde{F}^{\prime}(t)$ is larger than $r^{-}$. Therefore Propositions 1.14 and 1.4 yield:

$$
\left\|D f\left(x_{k}\right)^{-1} D f\left(x_{0}\right)\right\| \leq-\frac{1}{\tilde{F}^{\prime}\left(t_{k}\right)} .
$$

On the other hand, by definition of $x_{k}$, one can write:

$$
D f\left(x_{0}\right)^{-1} f\left(x_{k}\right)=D f\left(x_{0}\right)^{-1}\left(f\left(x_{k}\right)-f\left(x_{k-1}\right)-D f\left(x_{k-1}\right)\left(x_{k}-x_{k-1}\right)\right),
$$

so that a second order Taylor formula with integral remainder gives:

$D f\left(x_{0}\right)^{-1} f\left(x_{k}\right)=$

$$
\int_{0}^{1}(1-\tau) D f\left(x_{0}\right)^{-1} D^{2} f\left(x_{k-1}+\tau\left(x_{k}-x_{k-1}\right)\right)\left(x_{k}-x_{k-1}\right)^{2} d \tau .
$$

By Proposition 1.3, one has $\left[D f\left(x_{0}\right)^{-1} D^{2} f\right]_{x_{0}} \leq F^{\prime \prime}$ and then, using Proposition 1.4, we deduce: $\left\|D f\left(x_{0}\right)^{-1} f\left(x_{k}\right)\right\| \leq$

$$
\int_{0}^{1}(1-\tau) \tilde{F}^{\prime \prime}\left(\tau t_{k}+(1-\tau) t_{k-1}\right)\left(t_{k}-t_{k-1}\right)^{2} d \tau=\tilde{F}\left(t_{k}\right) .
$$

Combining (12) and (13) leads to:

$$
\begin{aligned}
\left\|x_{k+1}-x_{k}\right\| & \leq\left\|D f\left(x_{k}\right)^{-1} f\left(x_{k}\right)\right\| \\
& \leq\left\|D f\left(x_{k}\right)^{-1} D f\left(x_{0}\right)\right\|\left\|D f\left(x_{0}\right)^{-1} f\left(x_{k}\right)\right\| \\
& \leq-\tilde{F}\left(t_{k}\right) / \tilde{F}^{\prime}\left(t_{k}\right)=t_{k+1}-t_{k},
\end{aligned}
$$

which gives (10). We deduce that the sequence $\left(x_{k}\right)_{k \in \mathbb{N}}$ converges to a limit that we write $\zeta$. By construction, we have $f(\zeta)=0$. From (11), we get $\zeta \in \bar{B}\left(x_{0}, r^{-}\right)$, thus $\zeta$ is a simple zero.

Next, Part (b) follows from (13) this way:

$$
\begin{aligned}
\left\|D f\left(x_{0}\right)^{-1} f\left(x_{k}\right)\right\| & \leq \tilde{F}\left(t_{k}\right)=-\tilde{F}^{\prime}\left(t_{k}\right)\left(t_{k+1}-t_{k}\right) \\
& \leq-\tilde{F}^{\prime}(0)\left(t_{k+1}-t_{k}\right)=t_{k+1}-t_{k} .
\end{aligned}
$$

Lastly, Part (c) comes from

$$
\left\|x_{k}-\zeta\right\| \leq \sum_{i \geq k}\left\|x_{i}-x_{i+1}\right\| \leq \sum_{i \geq k}\left(t_{i+1}-t_{i}\right)=r^{-}-t_{k} .
$$


It remains to show that $\zeta$ is the only zero of $f$ in $\bar{B}\left(x_{0}, r\right)$. Let $\zeta^{\prime}$ denote another zero in this ball. Let $R>r$ be smaller than the radius of convergence of $F$ such that $\tilde{F}(R) \leq 0$. We introduce $\theta:=\left\|x_{0}-\zeta^{\prime}\right\| / R<1$ and claim

$$
\left\|x_{k}-\zeta^{\prime}\right\| \leq \theta^{2^{k}}\left(R-t_{k}\right), \text { for all } k \geq 0,
$$

from which immediately follows $\zeta=\zeta^{\prime}$. We prove this claim by induction on $k$. The case $k=0$ follows from the definition of $\theta$. Assume that (14) holds at $k \geq 0$, by construction, one can write:

$$
\begin{aligned}
x_{k+1}-\zeta^{\prime} & =-D f\left(x_{k}\right)^{-1}\left(f\left(x_{k}\right)+D f\left(x_{k}\right)\left(\zeta^{\prime}-x_{k}\right)\right) \\
& =D f\left(x_{k}\right)^{-1} \int_{0}^{1}(1-\tau) D^{2} f\left(x_{k}+\tau\left(\zeta^{\prime}-x_{k}\right)\right)\left(\zeta^{\prime}-x_{k}\right)^{2} d \tau,
\end{aligned}
$$

using again Taylor formula with integral remainder and $f\left(\zeta^{\prime}\right)=0$. Using the induction hypothesis and Proposition 1.14, we deduce:

$$
\left\|x_{k+1}-\zeta^{\prime}\right\| \leq-\frac{\theta^{2^{k+1}}}{\tilde{F}^{\prime}\left(t_{k}\right)} \int_{0}^{1}(1-\tau) \tilde{F}^{\prime \prime}\left(t_{k}+\tau \theta^{2^{k}}\left(R-t_{k}\right)\right)\left(R-t_{k}\right)^{2} d \tau .
$$

Since $\tilde{F}^{\prime \prime}$ is increasing, we can omit the factor $\theta^{2^{k}-1}$ under the integral. Using $\tilde{F}(R) \leq 0$, we deduce:

$$
\begin{aligned}
\left\|x_{k+1}-\zeta^{\prime}\right\| & \leq-\theta^{2^{k+1}} \frac{\tilde{F}(R)-\tilde{F}\left(t_{k}\right)-\tilde{F}^{\prime}\left(t_{k}\right)\left(R-t_{k}\right)}{\tilde{F}^{\prime}\left(t_{k}\right)} \\
& \leq \theta^{2^{k+1}} \frac{\tilde{F}\left(t_{k}\right)+\tilde{F}^{\prime}\left(t_{k}\right)\left(R-t_{k}\right)}{\tilde{F}^{\prime}\left(t_{k}\right)}=\theta^{2^{k+1}}\left(R-t_{k+1}\right) .
\end{aligned}
$$

Specializing $F$ to $F\left(f, x_{0} ;\right.$. ), we recover the following result by calculating an explicit expression for $t_{k}$ :

Corollary 1.18. ( $\alpha$-theorem) [55] If $\bar{B}:=\bar{B}\left(x_{0},(1+\sqrt{2} / 2) \beta\right) \subseteq U$ and $\alpha<3-2 \sqrt{2}$ then $f$ has exactly one simple zero $\zeta$ in $\bar{B}$ and the sequence $\left(x_{k}\right)_{k \in \mathbb{N}}$ recursively defined by $x_{k+1}:=N\left(f ; x_{k}\right)$ is well defined. In addition, $x_{k}$ belongs to $\bar{B}$ and for all $k \geq 0$ :

a. $\left\|x_{k}-x_{k+1}\right\| \leq q^{2^{k}-1} \beta$,

b. $\left\|D f\left(x_{0}\right)^{-1} f\left(x_{k}\right)\right\| \leq q^{2^{k}-1} \beta$,

c. $\left\|x_{k}-\zeta\right\| \leq q^{2^{k}-1} r^{-}$,

where $q:=\frac{2 r^{-}-\beta}{2 r^{+}-\beta}<1$ if $\alpha \neq 0$ and $q=0$ otherwise (in all cases we use the convention $q^{0}=1$ ).

Using (7), note that $q$ rewrites in terms of $\alpha$ only:

$$
q(\alpha):=\frac{4 \alpha}{\left(1-\alpha+\sqrt{1-6 \alpha+\alpha^{2}}\right)^{2}} .
$$

It follows that $q$ and $q / \alpha$ are continuous on $[0,3-2 \sqrt{2})$.

Proof. The case $\alpha=0$ is straightforward with the convention. We now assume $\alpha \neq 0$. It is classical to get an explicit formula of $t_{k}$ by means 
of introducing $s_{k}:=\frac{t_{k}-r^{-}}{t_{k}-r^{+}}$. Then, writing $\tilde{F}\left(f, x_{0} ; t\right)=2 \gamma \frac{\left(t-r^{-}\right)\left(t-r^{+}\right)}{1-\gamma t}$, we deduce

$$
\frac{\tilde{F}^{\prime}\left(f, x_{0} ; t\right)}{\tilde{F}\left(f, x_{0} ; t\right)}=\frac{1}{t-r^{-}}+\frac{1}{t-r^{+}}+\frac{\gamma}{1-\gamma t}
$$

and

$$
\begin{aligned}
s_{k+1} & =\frac{t_{k}-r^{-}-\frac{\tilde{F}\left(f, x_{0} ; t_{k}\right)}{\tilde{F}^{\prime}\left(f, x_{0} ; t_{k}\right)}}{t_{k}-r^{+}-\frac{\tilde{F}\left(f, x_{0} ; t_{k}\right)}{\tilde{F}^{\prime}\left(f, x_{0} ; t_{k}\right)}}=\frac{\left(t_{k}-r^{-}\right) \frac{\tilde{F}^{\prime}\left(f, x_{0} ; t_{k}\right)}{\tilde{F}\left(f, x_{0} ; t_{k}\right)}-1}{\left(t_{k}-r^{+}\right) \frac{\tilde{F}^{\prime}\left(f, x_{0} ; t_{k}\right)}{\tilde{F}\left(f, x_{0} ; t_{k}\right)}-1} \\
& =s_{k}^{2} \frac{1+\gamma \frac{t_{k}-r^{+}}{1-\gamma t_{k}}}{1+\gamma \frac{t_{k}-r^{-}}{1-\gamma t_{k}}}=s_{k}^{2} q_{s},
\end{aligned}
$$

where $q_{s}:=\frac{1-\gamma r^{+}}{1-\gamma r^{-}}$. From $s_{0}=r^{-} / r^{+}$and the definitions of $r^{-}$and $r^{+}$, it is easy to see that $\alpha<3-2 \sqrt{2}$ is equivalent to $q_{s} s_{0}<1$. Then, one can check $q=q_{s} s_{0}$ and an easy induction gives $s_{k}=q^{2^{k}-1} s_{0}$. It follows that $s_{k}$ converges quadratically to 0 and $t_{k}$ converges quadratically to $r^{-}$, since $t_{k}=r^{-\frac{1-s_{k} / s_{0}}{1-s_{k}}}$. In order to prove Part (a) we write

$$
t_{k+1}-t_{k}=\frac{r^{+}-r^{-}}{1-s_{k}} \frac{s_{k}-s_{k+1}}{1-s_{k+1}}
$$

and then deduce

$$
\begin{aligned}
\frac{t_{k+1}-t_{k}}{t_{1}-t_{0}} & =\frac{s_{k}-s_{k+1}}{s_{0}-s_{1}} \frac{1-s_{0}}{1-s_{k}} \frac{1-s_{1}}{1-s_{k+1}} \\
& =\frac{s_{k}}{s_{0}} \frac{1-q_{s} s_{k}}{1-q_{s} s_{0}} \frac{1-s_{0}}{1-s_{k}} \frac{1-q_{s} s_{0}^{2}}{1-q_{s} s_{k}^{2}} \leq \frac{s_{k}}{s_{0}},
\end{aligned}
$$

the last inequality follows from $q_{s} \leq 1$. Parts (a) and (b) follow from the previous theorem. Lastly, we deduce from Part (c) of the previous theorem:

$$
\left\|x_{k}-\zeta\right\| \leq r^{-}-t_{k}=r^{-} \frac{s_{k}}{s_{0}} \frac{1-s_{0}}{1-s_{k}} \leq r^{-} \frac{s_{k}}{s_{0}} .
$$

\section{Local Compositional Inverse}

The first main ingredient of our location and approximation algorithms is a quantitative version of the local compositional inverse function theorem. This section is devoted to the following theorem. We do not use our main notation from the introduction yet.

Theorem 2.1. Let $U \subseteq \mathbb{C}^{n}$ be an open subset, $f: U \rightarrow \mathbb{C}^{n}$ be an analytic map and $\zeta \in U$ be such that $D f(\zeta)$ is invertible. Let $\sigma \geq\left\|D f(\zeta)^{-1}\right\|$, $\gamma \geq \gamma(f ; \zeta), B_{f}:=B\left(\zeta, \frac{1-\sqrt{2} / 2}{\gamma}\right)$ and assume $B_{f} \subseteq U$. Then there exists a unique map $g$ with the following properties:

a. $g$ is defined and analytic in

$$
B_{g}:=B\left(f(\zeta), \frac{1}{(3+2 \sqrt{2}) \sigma \gamma}\right)
$$

b. $g\left(B_{g}\right) \subseteq B_{f}$;

c. $f \circ g(b)=b$, for all $b \in B_{g}$; 
d. For all $b \in B_{g}$ there exists only one $a \in B_{f}$ such that $f(a)=b$. In addition, we have $a=g(b)$ and $\|a-\zeta\| \leq(1+\sqrt{2} / 2) \beta(f-b ; \zeta) ;$

e. $[g-\zeta]_{f(\zeta)} \leq \frac{\sigma t}{1-(3+2 \sqrt{2}) \sigma \gamma t}$.

Proof. Let $b \in B_{g}, f_{b}(x)=f(x)-b$ defined on $B_{f}$. Then $\gamma\left(f_{b} ; \zeta\right)=\gamma(f ; \zeta) \leq$ $\gamma$ and

$$
\alpha\left(f_{b} ; \zeta\right) \leq \gamma \sigma\|f(\zeta)-b\|<3-2 \sqrt{2} .
$$

Hence Corollary 1.13 applies: $f_{b}(x)$ admits one simple zero $a$ in $B_{f}$, we define $g(b):=a$. By construction, $g$ is defined and analytic on $B_{g}$, hence Parts (b), (c) and (d) hold. Lastly, proving Part (e) is the goal of the rest of this section.

Remark that, according to the notation of this proof, Corollary $1.18 \mathrm{im}-$ plies that Newton's operator on $f_{b}$ converges quadratically from $\zeta$ to $a$. Except for Part (e), this theorem and the above proof are similar to [4, Chapter 8, Theorem 7]. It is also worth mentioning that Part (e) also implies [8, Corollary 3.4] in a straightforward way.

In order to prove the last statement (e), we now focus on the behaviour of majorant series under local compositional inversion. Then we deduce the claimed bound by specializing to geometric majorant series. The following results generalize and slightly improve those of [8, Section 3]. We also give different and simpler proofs. We start from the same proposition:

Proposition 2.2. [8, Theorem 3.2] Let $f$ be an analytic map from an open neighbourhood $U$ of a in $\mathbb{C}^{n}$ to $\mathbb{C}^{n}$ such that $\operatorname{Df}(a)=\mathrm{Id}$. Then $f$ is invertible in a neighbourhood of $f(a)$. Let $H \in t^{2} \mathbb{R}\{t\}$ be such that $[f-f(a)]_{a} \leq$ $t+H$. Let $F:=t-H$, and $F^{-1}$ denote the compositional inverse of $F$, then $\left[f^{-1}-a\right]_{f(a)} \leq F^{-1}$.

Proof. We refer to the proof of [8], based on Faà di Bruno's formula.

By considering inverses on the left and inverses on the right, we now relax the condition $D f(a)=\mathrm{Id}$.

Corollary 2.3. Let $f$ be an analytic map from an open neighbourhood $U$ of $a$ in $\mathbb{C}^{n}$ to $\mathbb{C}^{n}$. Assume that $D f(a)$ is invertible, then $f$ is invertible in a neighbourhood of $f(a)$. Let $g_{1}$ and $g_{2}$ be defined by

$$
g_{1}(x):=f\left(a+D f(a)^{-1} x\right)-f(a), \quad g_{2}(x):=D f(a)^{-1}(f(a+x)-f(a)) .
$$

For $i \in\{1,2\}$, let $H_{i} \in t^{2} \mathbb{R}\{t\}$ satisfy $\left[g_{i}\right]_{0} \leq t+H_{i}$. Define $F_{i}:=t-H_{i}$. Then

$$
\begin{aligned}
{\left[D f(a)\left(f^{-1}-a\right)\right]_{f(a)} } & \leq F_{1}^{-1}(t), \\
{\left[f^{-1} \circ D f(a)-a\right]_{D f(a)^{-1} f(a)} } & \leq F_{2}^{-1}(t) .
\end{aligned}
$$

Hence

$$
\left[f^{-1}-a\right]_{f(a)} \leq\left\|D f(a)^{-1}\right\| F_{1}^{-1}(t) \text { and }\left[f^{-1}-a\right]_{f(a)} \leq F_{2}^{-1}\left(\left\|D f(a)^{-1}\right\| t\right) .
$$


Proof. Since $D g_{i}(0)=\mathrm{Id}, i \in\{1,2\}$, we apply the previous proposition with $g_{i}$ and $H_{i}$ at 0 . Then we use the fact that

$$
f^{-1}(y)=a+D f(a)^{-1} g_{1}^{-1}(y-f(a))=a+g_{2}^{-1}\left(D f(a)^{-1}(y-f(a))\right) .
$$

Now we specialize this last corollary to the special case of geometric majorant series and obtain:

Corollary 2.4 (for Geometric majorant series). Let $f$ be an analytic map from an open neighbourhood $U$ of a in $\mathbb{C}^{n}$ to $\mathbb{C}^{n}$. Assume that $\operatorname{Df}(a)$ is invertible.

a. If $[f-f(a)]_{a} \leq \lambda t /(1-\rho t)$ then

$$
\left[D f(a)\left(f^{-1}-a\right)\right]_{f(a)} \leq \frac{t}{1-\vartheta \rho\left\|D f(a)^{-1}\right\| t} .
$$

b. If $\left[D f(a)^{-1}(f-f(a))\right]_{a} \leq \frac{\lambda\left\|D f(a)^{-1}\right\| t}{1-\rho t}$ then

$$
\left[f^{-1} \circ D f(a)-a\right]_{D f(a)^{-1} f(a)} \leq \frac{t}{1-\vartheta \rho t} .
$$

In both cases, $\vartheta$ denotes the largest root of $P(x):=1-2\left(1+2\left\|D f(a)^{-1}\right\| \lambda\right) x+$ $x^{2}$.

Observe that the defining polynomial $P$ for $\vartheta$ admits two nonnegative real roots and that $\vartheta$ is larger than one.

Proof. For Part (a), we apply the previous corollary with $H_{1}=\mu \bar{\rho} t^{2} /(1-\bar{\rho} t)$, where $\mu=\left\|D f(a)^{-1}\right\| \lambda$ and $\bar{\rho}=\rho\left\|D f(a)^{-1}\right\|$. We are then led to study the inverse $G$ of

$$
F=t-\frac{\mu \bar{\rho} t^{2}}{1-\bar{\rho} t}
$$

This inverse is given by the explicit formula

$$
G=\frac{1+\bar{\rho} t-\sqrt{1-2(1+2 \mu) \bar{\rho} t+\bar{\rho}^{2} t^{2}}}{2 \bar{\rho}(1+\mu)} .
$$

The rest of the proof is thus concentrated in the study of one specific map, which is performed in Lemma 2.6 below.

For Part (b) we apply the previous corollary with $H_{2}=\mu \rho t^{2} /(1-\rho t)$ and the rest is similar to Part (a).

Let us conclude:

Proof of Part (e) of Theorem 2.1. The proof follows from Part (b) of the previous corollary applied with $\lambda=1 /\left\|D f(a)^{-1}\right\|, \rho=\gamma(f ; a)$ and $\vartheta=$ $3+2 \sqrt{2}$.

Before coming to technical lemmas, we give two examples illustrating the sharpness of the previous corollary.

First, we consider $f=t-t^{2} /(1-t)$. Then Part (a) or (b) of the corollary applies with $\lambda=\rho=f^{\prime}(0)=1$ and gives the bound $\left[f^{-1}\right]_{0} \leq t /(1-\vartheta t)=$ : $B(t)$, with $\vartheta=3+2 \sqrt{2}$. The value of $\vartheta$ in the denominator is optimal. Indeed, from the explicit formula for $G$ above, we can use Darboux's theorem [19] to deduce that as $n$ tends to infinity, the $n$th coefficient of the 
Taylor expansion of $G$ at the origin behaves like $\kappa \vartheta^{n} n^{-3 / 2}$ for some constant $\kappa$. A smaller constant $\vartheta^{\prime}<\vartheta$ in the denominator of $B$ would lead to an asymptotic bound of order $\vartheta^{\prime n}$, incompatible with the actual behaviour. Concerning the numerator of $B$, it is also optimal since the first coefficient of $f^{-1}$ is 1 .

Our second example is $f=t-2 t^{2} /(1-t)$. As in the previous example, $\rho=f^{\prime}(0)=1$, but now, $\lambda=2$. The value of $\vartheta$ given by Part (a) is $5+$ $2 \sqrt{6} \approx 9.90$, which again is optimal with respect to the asymptotic behaviour of the Taylor coefficients, while the numerator is again sharp for the first coefficient. Applying Part (b) yields $\gamma\left(f^{-1} ; 0\right) \leq(3+2 \sqrt{2}) 2 \approx 11.66$, which illustrates the gain in dealing with a majorant geometric series given by two real numbers instead of only the gamma estimate.

Let us now complete the proof of Corollary 2.4. The following lemma is probably classical, although we could not find a reference to it.

Lemma 2.5. Let $P(t):=\sum_{i=0}^{p} a_{i} t^{i}$ be a polynomial with real coefficients such that $a_{0}>0$ and the Taylor series of $1 / P$ at the origin has nonnegative coefficients. Let $u_{n}$ be a sequence satisfying the inequality

$$
\mathcal{R}(u ; n):=\sum_{i=0}^{p} a_{p-i} u_{n+i} \leq 0, \quad n \geq 0 .
$$

Let $v_{n}$ be a sequence defined by $v_{i}=u_{i}$, for $0 \leq i<p$ and $\mathcal{R}(v ; n)=0$ for $n \geq 0$. Then $u_{n} \leq v_{n}$ for all $n \geq 0$.

Proof. Let $U(t):=\sum u_{n} t^{n}$ (resp. $V(t):=\sum v_{n} t^{n}$ ) be the generating series of the sequence $u_{n}$ (resp. $v_{n}$ ). By multiplying (16) by $t^{n+p}$ and summing over $n$, we get an inequality

$$
P(t) U(t) \leq P(t) V(t) .
$$

Observe that $P(t) V(t)$ is a polynomial of degree at most $p-1$. Since $1 / P$ has nonnegative coefficients, Part (d) of Proposition 1.1 allows to multiply both sides of the inequality by $1 / P$. Extracting coefficients of $t^{n}$ on both sides then concludes the proof.

Lemma 2.6. Let $P(t)=1-2 \nu t+t^{2}$, with $\nu>1$ and $G(t)=\frac{1+t-\sqrt{P(t)}}{1+\nu}$. Then

$$
[G]_{0} \leq \frac{t}{1-\vartheta t}
$$

where $\vartheta$ is the largest root of $P$.

Proof. The function $G$ satisfies the linear differential equation

$$
P(t) G^{\prime}(t)+(\nu-t) G(t)=1-t .
$$

It follows that the sequence $\left\{u_{n}\right\}$ of its Taylor coefficients at the origin satisfies the linear recurrence equation

$$
u_{n}=\left(2-\frac{3}{n}\right) \nu u_{n-1}-\left(1-\frac{3}{n}\right) u_{n-2}, \quad n \geq 3,
$$

with initial conditions $u_{0}=0, u_{1}=1, u_{2}=(\nu-1) / 2$. We now prove that

$$
0<u_{n}<2 \nu u_{n-1}-u_{n-2}, \quad n \geq 4 .
$$


To this aim, we first prove by induction that $u_{n}>u_{n-1}$ for $n \geq 3$. For $n=3$, we have $u_{3}=\nu u_{2}$ from (17) which gives the desired inequality since $\nu>1$ and $u_{2}>0$. For $n \geq 3$, let $v_{n}=u_{n} / u_{n-1}$ and assume $v_{n-1}>1$, then (17) implies

$$
v_{n}=\left(2-\frac{3}{n}\right) \nu-\frac{1-3 / n}{v_{n-1}} \geq 1+(2-3 / n)(\nu-1)>1 .
$$

From $u_{n} / u_{n-1}>1$, the sign of $u_{n}$ follows ( $u_{2}$ being positive) and since $\nu>$ 1 , we also get $v_{n}<2 \nu-1 / v_{n-1}$ for $n \geq 4$, whence the second part of the desired inequality (18). The polynomial $P$ can be written $(1-\vartheta t)(1-t / \vartheta)$ with $\vartheta$ its largest positive root. It follows that the Taylor series of $1 / P$ at the origin has nonnegative coefficients. We can therefore apply the previous lemma to the sequence $u_{n+2}$, which gives

$$
\frac{G(t)-u_{0}-u_{1} t}{t^{2}} \leq \frac{u_{2}+\left(u_{3}-2 \nu u_{2}\right) t}{P(t)} .
$$

Isolating $G(t) / t$ and computing a partial fraction expansion of the righthand side gives

$$
\frac{G(t)}{t} \leq 1+\frac{\nu(1-\nu)}{2}+\frac{C(\vartheta)}{1-\vartheta t}+\frac{C(1 / \vartheta)}{1-t / \vartheta},
$$

with $C(\vartheta)=\frac{\nu-1}{2}(\nu-\vartheta / 2)$. Now, obviously (since $\left.\vartheta>1\right)$

$$
\frac{1}{1-t / \vartheta}-1 \leq \frac{\vartheta^{-2}}{1-\vartheta t}-\vartheta^{-2}
$$

which makes it possible to bound the last summand in terms of the second one. A straightforward computation gives $C(\vartheta)+C(1 / \vartheta) / \vartheta^{2}=(\nu-1) /(2 \vartheta)$. The proof is concluded by showing that this last quantity is smaller than 1 . Indeed, by writing $\vartheta=\nu+\sqrt{\nu^{2}-1}$ and dividing by $\nu-1$, we see that $\vartheta /(\nu-1)>2$, which is sufficient. The numerator 1 is then dictated by the first coefficient $u_{1}=1$.

\section{Reduction to One Variable}

This section contains the last ingredients that are used in the next sections to reduce the location and approximation techniques from several variables to one variable, as explained in the introduction. We start by recalling the main result we shall use on location of clusters of analytic functions from $[12$, Section 1]. Then, as required by the univariate approximation algorithm of $\left[12\right.$, Section 4], we provide a function $\mathcal{B}_{m, l}$ that computes approximations of $\beta_{m, l}$. Next, we prove formula (4) from the introduction, that relates $D_{y}^{l} h$ to $g^{[l]}$ and, lastly, we provide bounds on translation with respect to $z$ of point estimates of maps $h(z,$.$) .$

3.1. Clusters of Zeroes of Univariate Functions. For the sake of completeness we recall the following result.

Theorem 3.1. [12, Corollary 1.8] Let $f$ denote an analytic function defined on a connected open subset $U \subseteq \mathbb{C}$, let $m \geq 1$ be an integer, $l \in\{0, \ldots, m-$ 
$1\}, z \in U$ be such that $f^{(m)}(z) \neq 0$,

$$
\frac{m-l}{m} \frac{m+1}{m+1-l} \alpha_{m, l}(f ; z) \leq 1 / 9
$$

and $\bar{B}\left(z, 3 \frac{m-l}{m} \beta_{m, l}(f ; z)\right) \subseteq U$. Then, $f^{(l)}$ has $m-l$ zeroes, counting multiplicities, in $\bar{B}\left(z, 3 \frac{m-l}{m} \beta_{m, l}(f ; z)\right)$ and $\bar{B}\left(z, \frac{m+1-l}{3(m+1) \gamma_{m}(f ; z)}\right) \cap U$.

Let us recall from [12, Section 2] that if $\alpha_{m, l}(f ; z)$ is sufficiently small and if $z$ is in the convex hull of the cluster of zeroes of $f^{(l)}$ located by the previous proposition then the diameter of this cluster is about $\beta_{m, l}(f ; z)$. Roughly speaking, this means that one can confound the diameter of this cluster and $\beta_{m, l}(f ; z)$, for any point in the convex hull of the cluster. This is why we focus on such quantities $\beta_{m, l}(f ; z)$ to approximate clusters in Section 5.

From [12, Section 4.1], we recall bounds on translation of $\alpha_{m, l}, \beta_{m, l}$ and $\gamma_{m}$ estimates that will be particularly useful in practice in our last section.

Proposition 3.2. [12, Proposition 4.3] Assume that $U$ is connected, let $\zeta \in U, m \geq 1$ be an integer such that $f^{(m)}(\zeta) \neq 0$ and $l \in\{0, \ldots, m-1\}$. Let $\gamma_{m}:=\gamma_{m}(f ; \zeta)$ and $\beta_{m, l}:=\beta_{m, l}(f ; \zeta)$, for short. Let $z \in U, r:=|z-\zeta|$ be such that $u:=\gamma_{m}(f ; \zeta) r<1-(1 / 2)^{1 /(m+1)}$, then $f^{(m)}(z) \neq 0$ and

$$
\begin{aligned}
& \text { a. } \alpha_{m, l}(f ; z) \leq \frac{1}{\psi_{m}(u)^{2}}\left(\alpha_{m, l}(1-u)^{\frac{l+1}{m-l}}+(2 m-1) u\right) ; \\
& \text { b. } \beta_{m, l}(f ; z) \leq \frac{1-u}{\psi_{m}(u)}\left(\beta_{m, l}(1-u)^{\frac{l+1}{m-l}}+(2 m-1) r\right) ; \\
& \text { c. } \gamma_{m}(f, z) \leq \frac{\gamma_{m}}{\psi_{m}(u)(1-u)} ; \\
& \text { d. }\left|\frac{f^{(m)}(\zeta)}{f^{(m)}(z)}\right| \leq \frac{(1-u)^{m+1}}{\psi_{m}(u)} ; \\
& \text { e. }\left|\frac{f^{(m)}(z)}{f^{(m)}(\zeta)}\right| \leq \frac{1}{(1-u)^{m+1}} .
\end{aligned}
$$

3.2. Approximation of $\beta_{m, l}$. Let $U$ be a connected open subset of $\mathbb{C}$, in this subsection $f$ denotes an analytic function defined on $U$. Let $x_{0}$ and $x_{1}$ be two points in $U, m \geq 1$ be an integer such that $f^{m}\left(x_{0}\right) \neq 0$ and let $l \in\{0, \ldots, m-1\}$. We show that $\beta_{m, l}\left(f ; x_{1}\right)$ can be approximated from the sole knowledge of a truncated Taylor expansion of $f$ at $x_{0}$ and upper bounds on $\gamma_{m}(f ;$.$) at x_{0}$ and $x_{1}$. We introduce the following functions, that will be used in Section 5:

$$
\begin{gathered}
\mathcal{B}_{m, l}\left(f, x_{0} ; x_{1}\right):=\beta_{m, l}\left(p ; x_{1}\right), \quad v_{m, l}:=\min \left(1-\left(\frac{1}{2}\right)^{\frac{1}{m+1}}, \frac{1}{2 m-l}\right), \\
\tau_{m, l, 0}(v):=\frac{(2 m-l)^{2}(1-v)}{\psi_{m}(v)}, \quad \tau_{m, l, 1}(v):=1+\frac{(2 m-l) v}{\psi_{m}(v)},
\end{gathered}
$$

where $p$ denotes the unique polynomial of degree at most $2 m-l-1$ such that $f(x)-p(x) \in \mathcal{O}_{x_{0}}\left(\left(x-x_{0}\right)^{2 m-l}\right)$. These objects satisfy the requirements stated in [12, Section 4.2], namely: 
Proposition 3.3. Let $r:=\left|x_{0}-x_{1}\right|, \bar{\gamma}_{m} \geq \max \left(\gamma_{m}\left(f ; x_{0}\right), \gamma_{m}\left(f ; x_{1}\right)\right), v=$ $\bar{\gamma}_{m} r$, if $v<v_{m, l}$ then

$$
\begin{aligned}
\mathcal{B}_{m, l}\left(f, x_{0} ; x_{1}\right) & \leq \tau_{m, l, 1}(v) \beta_{m, l}\left(f ; x_{1}\right)+\tau_{m, l, 0}(v) \bar{\gamma}_{m} r^{2}, \\
\beta_{m, l}\left(f ; x_{1}\right) & \leq \tau_{m, l, 1}(v) \mathcal{B}_{m, l}\left(f, x_{0} ; x_{1}\right)+\tau_{m, l, 0}(v) \bar{\gamma}_{m} r^{2} .
\end{aligned}
$$

Proof. It suffices to set $i=2 m-l$ in the next lemma.

Lemma 3.4. Let $i \geq m+1$ be an integer and $p(y)$ denote the unique polynomial of degree at most $i-1$ such that $f(x)-p(x) \in \mathcal{O}_{x_{0}}\left(\left(x-x_{0}\right)^{i}\right)$. Let $r:=\left|x_{1}-x_{0}\right|, v:=\gamma_{m}\left(f ; x_{0}\right) r$ and assume $v<1-(1 / 2)^{1 /(m+1)}$ and $i v<1$, then $\left|\beta_{m, l}\left(f ; x_{1}\right)-\beta_{m, l}\left(p ; x_{1}\right)\right| \leq$

$$
\frac{(i v)^{\frac{i-m}{m-l}}}{\psi_{m}(v)}\left(\min \left(\beta_{m, l}\left(f ; x_{1}\right), \beta_{m, l}\left(p ; x_{1}\right)\right)+(1-v) i r\right) .
$$

Proof. Let $\gamma_{m}:=\gamma_{m}\left(f ; x_{0}\right)$ and $\sigma_{m}:=m ! /\left|f^{(m)}\left(x_{0}\right)\right|=m ! /\left|p^{(m)}\left(x_{0}\right)\right|$. We introduce the majorant series

$$
R:=\frac{\gamma_{m}^{i-m} t^{i}}{1-\gamma_{m} t}
$$

so that $\sigma_{m}[f-p]_{x_{0}} \leq R$. Let $l \leq k \leq m$. By [12, Lemma 4.1], one has

$$
\frac{R^{(k)}}{k !} \leq \frac{\gamma_{m}^{i-m}\left(\begin{array}{c}
i \\
k
\end{array}\right) t^{i-k}}{\left(1-\gamma_{m} t\right)^{k+1}} \leq \frac{\left(i \gamma_{m} t\right)^{i-m}(i t)^{m-k}}{\left(1-\gamma_{m} t\right)^{k+1}}
$$

Observe that $\gamma_{m}\left(p ; x_{0}\right) \leq \gamma_{m}$ and therefore both $f^{(m)}\left(x_{1}\right)$ and $p^{(m)}\left(x_{1}\right)$ do not vanish, according to Proposition 3.2, and:

$$
\left|\frac{m !}{\sigma_{m} p^{(m)}\left(x_{1}\right)}\right| \leq \frac{(1-v)^{m+1}}{\psi_{m}(v)}, \quad\left|\frac{m !}{\sigma_{m} f^{(m)}\left(x_{1}\right)}\right| \leq \frac{(1-v)^{m+1}}{\psi_{m}(v)}
$$

Then we start with:

$$
\begin{aligned}
\left|\frac{m ! f^{(k)}\left(x_{1}\right)}{k ! f^{(m)}\left(x_{1}\right)}-\frac{m ! p^{(k)}\left(x_{1}\right)}{k ! p^{(m)}\left(x_{1}\right)}\right| \leq & \left|\frac{m ! f^{(k)}\left(x_{1}\right)}{k ! p^{(m)}\left(x_{1}\right)}-\frac{m ! p^{(k)}\left(x_{1}\right)}{k ! p^{(m)}\left(x_{1}\right)}\right| \\
& +\left|\frac{m ! f^{(k)}\left(x_{1}\right)}{k ! p^{(m)}\left(x_{1}\right)}-\frac{m ! f^{(k)}\left(x_{1}\right)}{k ! f^{(m)}\left(x_{1}\right)}\right| .
\end{aligned}
$$

Using majorant series evaluation via Proposition 1.4, we bound the first term of the right-hand side of the last inequality:

$$
\begin{aligned}
\left|\frac{m ! f^{(k)}\left(x_{1}\right)}{k ! p^{(m)}\left(x_{1}\right)}-\frac{m ! p^{(k)}\left(x_{1}\right)}{k ! p^{(m)}\left(x_{1}\right)}\right| & \leq\left|\frac{m !}{p^{(m)}\left(x_{1}\right)}\right|\left|\frac{f^{(k)}\left(x_{1}\right)}{k !}-\frac{p^{(k)}\left(x_{1}\right)}{k !}\right| \\
& \leq\left|\frac{m !}{\sigma_{m} p^{(m)}\left(x_{1}\right)}\right|\left|\frac{R^{(k)}(r)}{k !}\right| \\
& \leq \frac{(1-v)^{m+1}}{\psi_{m}(v)} \frac{(i v)^{i-m}(i r)^{m-k}}{(1-v)^{k+1}} \\
& \leq \frac{(1-v)^{m-k}}{\psi_{m}(v)}(i v)^{i-m}(i r)^{m-k} .
\end{aligned}
$$


As for the second term we get:

$$
\begin{aligned}
\left|\frac{m ! f^{(k)}\left(x_{1}\right)}{k ! p^{(m)}\left(x_{1}\right)}-\frac{m ! f^{(k)}\left(x_{1}\right)}{k ! f^{(m)}\left(x_{1}\right)}\right| & \leq\left|\frac{m ! f^{(k)}\left(x_{1}\right)}{k ! f^{(m)}\left(x_{1}\right)}\right|\left|\frac{m !}{p^{(m)}\left(x_{1}\right)}\right|\left|\frac{f^{(m)}\left(x_{1}\right)}{m !}-\frac{p^{(m)}\left(x_{1}\right)}{m !}\right| \\
& \leq \beta_{m, l}\left(f ; x_{1}\right)^{m-k}\left|\frac{m !}{\sigma_{m} p^{(m)}\left(x_{1}\right)}\right|\left|\frac{R^{(m)}(r)}{m !}\right| \\
& \leq \beta_{m, l}\left(f ; x_{1}\right)^{m-k} \frac{(1-v)^{m+1}}{\psi_{m}(v)} \frac{(i v)^{i-m}}{(1-v)^{m+1}} \\
& \leq \beta_{m, l}\left(f ; x_{1}\right)^{m-k} \frac{(i v)^{i-m}}{\psi_{m}(v)}
\end{aligned}
$$

Then using the assumption $i v<1$, we conclude:

$$
\left|\beta_{m, l}\left(f ; x_{1}\right)-\beta_{m, l}\left(p ; x_{1}\right)\right| \leq \frac{(i v)^{\frac{i-m}{m-l}}}{\psi_{m}(v)}\left(\beta_{m, l}\left(f ; x_{1}\right)+(1-v) i r\right),
$$

which is the first half of the claimed inequality. The second half is obtained in a similar way, starting from (19) and exchanging the roles of $f$ and $p$.

3.3. Deflated Maps. From now on, we use main notation, given in the introduction. For any integer $l \geq 0$, in this text, we study the $l$ th deflated map $\left(f, g^{[l]}\right)$ obtained from $(f, g)$ according to the following recursive formal definition:

$$
g^{[0]}=g, \quad g^{[l+1]}=\frac{\operatorname{det}\left(D\left(f, g^{[l]}\right)\right)}{\operatorname{det}\left(D_{x} f\right)}, \text { for } l \geq 0,
$$

where det denotes the determinant map, the implicit basis being considered is $(x, y)$. For any $\left(x_{0}, y_{0}\right)$ such that $D_{x} f\left(x_{0}, y_{0}\right)$ is invertible, the relation with $h\left(f, g, x_{0}, y_{0} ; z, y\right)$ is as follows.

Lemma 3.5. Let $l \geq 0,\left(x_{0}, y_{0}\right) \in U$ be such that $D_{x} f\left(x_{0}, y_{0}\right)$ is invertible and $z_{0}:=D_{x} f\left(x_{0}, y_{0}\right)^{-1} f\left(x_{0}, y_{0}\right)$. The following relation holds in a neighborhood of $\left(z_{0}, y_{0}\right)$ :

$$
D_{y}^{l} h\left(f, g, x_{0}, y_{0} ; z, y\right)=g^{[l]} \circ \Phi\left(f, x_{0}, y_{0} ; z, y\right) .
$$

Proof. For short, we let

$$
h(z, y):=h\left(f, g, x_{0}, y_{0} ; z, y\right), \Phi(z, y):=\Phi\left(f, x_{0}, y_{0} ; z, y\right) .
$$

Let $X$ denote the first $n-1$ coordinates of $D_{y} \Phi(z, y)$ and $Y$ the last one. By construction, we have $Y=1$ and $D_{x} f\left(x_{0}, y_{0}\right)^{-1} f \circ \Phi(z, y)=z$. In a neighborhood of $\left(z_{0}, y_{0}\right)$, differentiating this equality with respect to $y$ yields

$$
D_{x} f(\Phi(z, y)) X+D_{y} f(\Phi(z, y))=0 .
$$

On the other hand we have $D_{y} h(z, y)=D g(\Phi(z, y)) D_{y} \Phi(z, y)$, hence:

$$
\begin{aligned}
D_{y} h(z, y) & =D_{x} g(\Phi(z, y)) X+D_{y} g(\Phi(z, y)) \\
& =-D_{x} g(\Phi(z, y)) D_{x} f(\Phi(z, y))^{-1} D_{y} f(\Phi(z, y))+D_{y} g(\Phi(z, y)) .
\end{aligned}
$$

For $l=1$, the conclusion is a consequence of the classical Schur complement formula, that comes from mapping determinant on each factor of

$$
\left(\begin{array}{cc}
\operatorname{Id} & 0 \\
D_{x} g(\Phi(z, y)) & -1
\end{array}\right)\left(\begin{array}{cc}
D_{x} f(\Phi(z, y))^{-1} & 0 \\
0 & 1
\end{array}\right)\left(\begin{array}{ll}
D_{x} f(\Phi(z, y)) & D_{y} f(\Phi(z, y)) \\
D_{x} g(\Phi(z, y)) & D_{y} g(\Phi(z, y))
\end{array}\right)
$$




$$
=\left(\begin{array}{cc}
\text { Id } & D_{x} f(\Phi(z, y))^{-1} D_{y} f(\Phi(z, y)) \\
0 & -D_{y} h(z, y)
\end{array}\right) .
$$

An easy induction on $l$ concludes the proof.

From a practical point of view, and by means of classical computations with power series, starting from $\left(x_{0}, y_{0}\right)$, one can obtain truncated Taylor series expansions of $\Phi$ at $\left(z_{0}, y_{0}\right)$ in order to deduce such expansions for $h$ via this lemma.

3.4. $z$-Translation of Point Estimates. Here we focus on quantifying the variations of $\beta_{m, l}(h(z,) ; y$.$) and \gamma_{m}(h(z,) ; y$.$) when z$ varies, for fixed $y$, where $h$ denotes an analytic map from an open subset $U$ of $\mathbb{C}^{n-1} \times \mathbb{C}$ and with values in $\mathbb{C}$.

Proposition 3.6. Let $h(z, y)$ be an analytic map defined on a connected open neighborhood $U$ of $\left(z_{0}, y_{0}\right)$ to $\mathbb{C}$. Let $\lambda \geq 0$ and $\rho \geq 0$ be two real numbers satisfying $\left[h-h\left(z_{0}, y_{0}\right)\right]_{\left(z_{0}, y_{0}\right)} \leq \frac{\lambda t}{1-\rho t}$. Let $m \geq 1$ and $l \in\{0, \ldots, m-1\}$ be integers such that $\left|D_{y}^{m} h\left(z_{0}, y_{0}\right)\right| \neq 0$ and let $\sigma_{m} \geq m ! /\left|D_{y}^{m} h\left(z_{0}, y_{0}\right)\right|$. Let $z_{1}$ be such that $\left(z_{1}, y_{0}\right) \in U$ and

$$
\mu:=\frac{\rho}{1-\rho\left\|z_{1}-z_{0}\right\|}, \quad e:=\left((m+1) \sigma_{m} \frac{\lambda\left\|z_{1}-z_{0}\right\|}{1-\rho\left\|z_{1}-z_{0}\right\|}\right)^{1 / m} .
$$

If $\rho\left\|z_{1}-z_{0}\right\|<1$ and $\mu e<1$ then $\left|D_{y}^{m} h\left(z_{1}, y_{0}\right)\right| \neq 0$ and

a. $m ! /\left|D_{y}^{m} h\left(z_{1}, y_{0}\right)\right| \leq \frac{m ! /\left|D_{y}^{m} h\left(z_{0}, y_{0}\right)\right|}{1-(\mu e)^{m}}$ and $m ! /\left|D_{y}^{m} h\left(z_{0}, y_{0}\right)\right| \leq\left(1+(\mu e)^{m}\right) m ! /\left|D_{y}^{m} h\left(z_{1}, y_{0}\right)\right| ;$

b. $\beta_{m, l}\left(h\left(z_{1},.\right) ; y_{0}\right) \leq \frac{\beta_{m, l}\left(h\left(z_{0}, .\right) ; y_{0}\right)+\frac{m}{m+1} \mu^{\frac{l}{m-l}} e^{\frac{m}{m-l}}}{1-(\mu e)^{m}}$ and $\beta_{m, l}\left(h\left(z_{0},.\right) ; y_{0}\right) \leq\left(1+(\mu e)^{m}\right) \beta_{m, l}\left(h\left(z_{1},.\right) ; y_{0}\right)+\frac{m}{m+1} \mu^{\frac{l}{m-l}} e^{\frac{m}{m-l}} ;$

c. $\gamma_{m}\left(h\left(z_{1},.\right) ; y_{0}\right) \leq \frac{\gamma_{m}\left(h\left(z_{0}, .\right) ; y_{0}\right)+\frac{m+2}{m+1} \mu}{1-(\mu e)^{m}}$ and $\gamma_{m}\left(h\left(z_{0},.\right) ; y_{0}\right) \leq\left(1+(\mu e)^{m}\right) \gamma_{m}\left(h\left(z_{1},.\right) ; y_{0}\right)+\frac{m+2}{m+1} \mu$.

Proof. Let $r:=\left\|z_{1}-z_{0}\right\|$. If $\rho=0$ then $m=1$ and the proposition holds trivially. Now we assume $\rho>0$. Inequalities $\left\|D_{z}^{j} D_{y}^{k} h\left(z_{0}, y_{0}\right)\right\| \leq$ $\left\|D^{j+k} h\left(z_{0}, y_{0}\right)\right\|$, for all $j \geq 0$ and $k \geq 0$, rewrite into $\left[D_{y}^{k} h\left(., y_{0}\right)\right]_{z_{0}} \leq$ $\left[D^{k} h\right]_{\left(z_{0}, y_{0}\right)}$. Differentiating majorant series, thanks to Proposition 1.3, we obtain, for any $k \geq 1$ :

$$
\begin{aligned}
{\left[\frac{D_{y}^{k} h\left(., y_{0}\right)}{k !}-\frac{D_{y}^{k} h\left(z_{0}, y_{0}\right)}{k !}\right]_{z_{0}} } & \leq\left[\frac{D^{k} h}{k !}-\frac{D^{k} h\left(z_{0}, y_{0}\right)}{k !}\right]_{\left(z_{0}, y_{0}\right)} \\
& \leq \frac{\lambda \rho^{k-1}}{(1-\rho t)^{k+1}}-\lambda \rho^{k-1}
\end{aligned}
$$


Evaluating at $t=r$, using Proposition 1.4, we deduce:

$$
\begin{aligned}
\left|\frac{\left|D_{y}^{k} h\left(z_{1}, y_{0}\right)\right|}{k !}-\frac{\left|D_{y}^{k} h\left(z_{0}, y_{0}\right)\right|}{k !}\right| & \leq \frac{\lambda \rho^{k-1}}{(1-\rho r)^{k+1}}\left(1-(1-\rho r)^{k+1}\right) \\
& \leq \frac{(k+1) \lambda \rho^{k} r}{(1-\rho r)^{k+1}}=(k+1) \mu^{k} \frac{\lambda r}{1-\rho r}
\end{aligned}
$$

since $1-(1-\rho r)^{k+1}$ is an increasing concave function of $r$. Observe that this last inequality also holds for $k=0$. Rewriting the previous expressions in terms of $e$ and $\mu$, we find:

$$
\left|\frac{m !\left|D_{y}^{k} h\left(z_{1}, y_{0}\right)\right|}{k !\left|D_{y}^{m} h\left(z_{0}, y_{0}\right)\right|}-\frac{m !\left|D_{y}^{k} h\left(z_{0}, y_{0}\right)\right|}{k !\left|D_{y}^{m} h\left(z_{0}, y_{0}\right)\right|}\right| \leq \frac{k+1}{m+1} \mu^{k-m}(\mu e)^{m} .
$$

Letting $k:=m$ in this inequality we obtain:

$$
1-(\mu e)^{m} \leq\left|D_{y}^{m} h\left(z_{1}, y_{0}\right)\right| /\left|D_{y}^{m} h\left(z_{0}, y_{0}\right)\right| \leq 1+(\mu e)^{m},
$$

which yields Part (a). Combining (20) and (21), we deduce, for any $k \geq 0$ :

$$
\frac{m !\left|D_{y}^{k} h\left(z_{1}, y_{0}\right)\right|}{k !\left|D_{y}^{m} h\left(z_{1}, y_{0}\right)\right|} \leq \frac{\frac{m !\left|D_{y}^{k} h\left(z_{0}, y_{0}\right)\right|}{k !\left|D_{y}^{m} h\left(z_{0}, y_{0}\right)\right|}+\frac{k+1}{m+1} \mu^{k-m}(\mu e)^{m}}{1-(\mu e)^{m}}
$$

and

$$
\frac{m !\left|D_{y}^{k} h\left(z_{0}, y_{0}\right)\right|}{k !\left|D_{y}^{m} h\left(z_{0}, y_{0}\right)\right|} \leq\left(1+(\mu e)^{m}\right) \frac{m !\left|D_{y}^{k} h\left(z_{1}, y_{0}\right)\right|}{k !\left|D_{y}^{m} h\left(z_{1}, y_{0}\right)\right|}+\frac{k+1}{m+1} \mu^{k-m}(\mu e)^{m} .
$$

For short, we let $\beta_{m, l}:=\beta_{m, l}\left(h\left(z_{0},.\right) ; y_{0}\right)$ and $\gamma_{m}:=\gamma_{m}\left(h\left(z_{0},.\right) ; y_{0}\right)$. For Part (b), let us consider $l \leq k \leq m-1$, then (21) and (22) respectively lead to:

$$
\beta_{m, l}\left(h\left(z_{1}, .\right) ; y_{0}\right) \leq \frac{\beta_{m, l}+\mu^{-1} \sup _{l \leq k \leq m-1} b_{m, k}(\mu e)^{\frac{m}{m-k}}}{1-(\mu e)^{m}}
$$

and

$$
\beta_{m, l} \leq\left(1+(\mu e)^{m}\right) \beta_{m, l}\left(h\left(z_{1}, .\right) ; y_{0}\right)+\mu^{-1} \sup _{l \leq k \leq m-1} b_{m, k}(\mu e)^{\frac{m}{m-k}},
$$

where $b_{m, k}:=\left(\frac{k+1}{m+1}\right)^{\frac{1}{m-k}}$. Part (b) follows from $\sup _{l \leq k \leq m-1}(\mu e)^{\frac{m}{m-k}}=$ $(\mu e)^{\frac{m}{m-l}}$ and

$$
\sup _{l \leq k \leq m-1} b_{m, k}=\frac{m}{m+1} .
$$

This equality can be seen as a consequence of the concavity of the log function:

$$
\begin{aligned}
\log \left(b_{m, k}\right) & =\frac{\log (k+1)-\log (m+1)}{(m+1)-(k+1)} \\
& \leq \frac{\log (m)-\log (m+1)}{(m+1)-m}=\log \left(b_{m, m-1}\right)
\end{aligned}
$$


which is an equality for $k=m-1$. As for Part (c), calculations are very similar. Letting $k \geq m+1$, and using $\mu e<1$, inequalities (21) and (22) respectively lead to:

$$
\gamma_{m}\left(h\left(z_{1}, .\right) ; y_{0}\right) \leq \frac{\gamma_{m}+\mu \sup _{k \geq m+1} c_{m, k}}{1-(\mu e)^{m}}
$$

and

$$
\gamma_{m} \leq\left(1+(\mu e)^{m}\right) \gamma_{m}\left(h\left(z_{1}, .\right) ; y_{0}\right)+\mu \sup _{k \geq m+1} c_{m, k},
$$

where $c_{m, k}:=\left(\frac{k+1}{m+1}\right)^{\frac{1}{k-m}}$. Part (c) follows from

$$
\sup _{k \geq m+1} c_{m, k}=\frac{m+2}{m+1} .
$$

Again, this equality can be seen as a consequence of the concavity of the log function:

$$
\begin{aligned}
\log \left(c_{m, k}\right) & =\frac{\log (k+1)-\log (m+1)}{(k+1)-(m+1)} \\
& \leq \frac{\log (m+2)-\log (m+1)}{(m+2)-(m+1)}=\log \left(c_{m, m+1}\right) .
\end{aligned}
$$

\section{Cluster location}

In this section, we use our main notation stated in the introduction: $f: U_{f} \rightarrow \mathbb{C}^{n-1}$ and $g: U_{g} \rightarrow \mathbb{C}$ are analytic maps defined on maximal analyticity domains. We present a method for locating clusters of zeroes of the $l$ th deflated map $\left(f, g^{[l]}\right)$.

In order to perform this location around a given point $\left(x_{0}, y_{0}\right)$, we focus on locating zeroes of $D_{y}^{l} h\left(f, g, x_{0}, y_{0} ; 0,.\right)$ around $y_{0}$, which reduces to a univariate situation. Unfortunately, it is not possible to compute point estimates of $h$ at $\left(0, y_{0}\right)$ in general. We have to content ourselves with estimates at $\left(z_{0}^{\prime}, y_{0}\right)$, where $z_{0}^{\prime}:=D_{x} f\left(x_{0}, y_{0}\right)^{-1} f\left(x_{0}^{\prime}, y_{0}\right)$ is small enough for $x_{0}^{\prime}$ is obtained from $x_{0}$ by means of Newton's iteration. Our algorithm together with its main properties are presented in the following theorem.

Theorem 4.1. Let $f: U_{f} \rightarrow \mathbb{C}^{n-1}$ and $g: U_{g} \rightarrow \mathbb{C}$ be maximal analytic maps with $U_{f} \cap U_{g} \neq \emptyset$. Let $\left(x_{0}, y_{0}\right) \in U_{f} \cap U_{g}$ be such that $D_{x} f\left(x_{0}, y_{0}\right)$ is invertible. Let $\Sigma(x, y):=\Sigma\left(f, x_{0}, y_{0} ; x, y\right), h(z, y):=h\left(f, g, x_{0}, y_{0} ; z, y\right)$. Let $m \geq 1, l \in\{0, \ldots, m-1\}, \kappa$ be the first integer such that $2^{\kappa} \geq m-l$, $x_{0}^{\prime}:=N^{\kappa}\left(f\left(., y_{0}\right) ; x_{0}\right)$ and $z_{0}^{\prime}:=D_{x} f\left(x_{0}, y_{0}\right)^{-1} f\left(x_{0}^{\prime}, y_{0}\right)$.

Let $\lambda_{g}, \rho_{g}, \beta_{x}, \gamma_{x}, \sigma_{x}, \beta_{m, l}, \gamma_{m}, \sigma_{m}$ be given nonnegative real numbers. We introduce the following quantities:

$$
\begin{aligned}
& \lambda_{\Phi}:=\sigma_{x} ; \rho_{\Phi}:=(3+2 \sqrt{2}) \sigma_{x} \gamma_{x} \\
& \lambda:=\lambda_{g} \lambda_{\Phi} ; \rho:=\rho_{\Phi}+\lambda_{\Phi} \rho_{g} ; \\
& \bar{\lambda}:=\frac{\lambda}{\left(1-\rho\left(\beta_{x}+\left\|z_{0}^{\prime}\right\|\right)\right)^{2}} ; \bar{\rho}:=\frac{\rho}{1-\rho\left(\beta_{x}+\left\|z_{0}^{\prime}\right\|\right)} \\
& \bar{\mu}:=\frac{\bar{\rho}}{1-\bar{\rho}\left\|z_{0}^{\prime}\right\|} ; \bar{e}:=\left((m+1) \sigma_{m} \frac{\bar{\lambda}\left\|z_{0}^{\prime}\right\|}{1-\bar{\rho}\left\|z_{0}^{\prime}\right\|}\right)^{1 / m}
\end{aligned}
$$




$$
\begin{aligned}
\bar{\beta}_{m, l} & :=\frac{\beta_{m, l}+\frac{m}{m+1} \bar{\mu}^{\frac{l}{m-l}} \bar{e}^{\frac{m}{m-l}}}{1-(\bar{\mu} \bar{e})^{m}} ; \bar{\gamma}_{m}:=\frac{\gamma_{m}+\frac{m+2}{m+1} \bar{\mu}}{1-(\bar{\mu} \bar{e})^{m}} ; \\
r_{y}^{-} & :=3 \frac{m-l}{m} \bar{\beta}_{m, l} ; r_{y}^{+}:=\frac{m+1-l}{3(m+1) \bar{\gamma}_{m}} \\
r_{x}^{-} & :=\frac{\lambda_{\Phi}\left(\beta_{x}+r_{y}^{-}\right)}{1-\rho_{\Phi}\left(\beta_{x}+r_{y}^{-}\right)} .
\end{aligned}
$$

If

$\left(L_{1}\right)\left[g-g\left(x_{0}, y_{0}\right)\right]_{\left(x_{0}, y_{0}\right)} \leq \frac{\lambda_{g} t}{1-\rho_{g} t} ;$

$\left(L_{2}\right) \sigma_{x} \geq\left\|D \Sigma\left(x_{0}, y_{0}\right)^{-1}\right\| ;$

$\left(L_{3}\right) \beta_{x} \geq \beta\left(\Sigma-\left(0, y_{0}\right) ; x_{0}, y_{0}\right) ; \gamma_{x} \geq \gamma\left(\Sigma ; x_{0}, y_{0}\right)$;

$\left(L_{4}\right) \rho\left(\beta_{x}+\left\|z_{0}^{\prime}\right\|\right)<1$;

$\left(L_{5}\right)\left|D_{y}^{m} h\left(z_{0}^{\prime}, y_{0}\right)\right| \neq 0 ; \sigma_{m} \geq m ! /\left|D_{y}^{m} h\left(z_{0}^{\prime}, y_{0}\right)\right|$;

$\left(L_{6}\right) \beta_{m, l} \geq \beta_{m, l}\left(h\left(z_{0}^{\prime},.\right) ; y_{0}\right) ; \gamma_{m} \geq \gamma_{m}\left(h\left(z_{0}^{\prime},.\right) ; y_{0}\right)$;

$\left(L_{7}\right) \bar{\rho}\left\|z_{0}^{\prime}\right\|<1 ; \bar{\mu} \bar{e}<1$;

$\left(L_{8}\right) \frac{m-l}{m} \frac{m+1}{m+1-l} \bar{\beta}_{m, l} \bar{\gamma}_{m} \leq 1 / 9$

then $\left(f, g^{[l]}\right)$ has $m-l$ zeroes, counting multiplicities, in

$$
B_{\Sigma} \cap B_{g} \cap\left(\bar{B}\left(x_{0}, r_{x}^{-}\right) \times \bar{B}\left(y_{0}, r_{y}^{-}\right)\right)
$$

but also in

$$
B_{\Sigma} \cap B_{g} \cap\left(\mathbb{C}^{n-1} \times \bar{B}\left(y_{0}, r_{y}^{+}\right)\right),
$$

where

$$
B_{\Sigma}:=B\left(\left(x_{0}, y_{0}\right), \frac{1-\sqrt{2} / 2}{\gamma_{x}}\right), B_{g}:=B\left(\left(x_{0}, y_{0}\right), \frac{1}{\rho_{g}}\right) .
$$

Before entering the proof, let us explain the main idea and feature of the method. First observe that if $\left(x_{0}, y_{0}\right)=\zeta$ is a multiple zero of multiplicity $m$ then the theorem applies. By continuity, it follows that the process actually locates clusters of embedding dimension 1. Informally speaking, by construction, we shall see below in (27) that $z_{0}^{\prime}$ belongs to $\mathcal{O}\left(\beta_{x}^{m-l}\right)$, hence $\bar{e}^{\frac{m}{m-l}} \in \mathcal{O}\left(\beta_{x}\right)$. Therefore, we have $\bar{\beta}_{m, l} \in \mathcal{O}\left(\beta_{m, l}+\beta_{x}\right)$, which is the motivation for the definition of $\kappa$ above.

Proof of Theorem 4.1. Recall that $\Phi$ denotes the local inverse of $\Sigma$ and, for short, we let

$$
z_{0}:=D_{x} f\left(x_{0}, y_{0}\right)^{-1} f\left(x_{0}, y_{0}\right), \Phi(z, y):=\Phi\left(f, x_{0}, y_{0} ; z, y\right),
$$

$\phi(y)$ denotes the $n-1$ first coordinates of $\Phi(0, y)$ and we introduce:

$$
\begin{gathered}
B_{\Phi}:=B\left(\left(z_{0}, y_{0}\right), \frac{3-2 \sqrt{2}}{\sigma_{x} \gamma_{x}}\right), B_{h}:=B\left(\left(z_{0}, y_{0}\right), \frac{1}{\rho}\right) \\
\text { and } B_{\phi}:=B\left(y_{0}, \frac{1}{\rho}-\beta_{x}\right) .
\end{gathered}
$$


From $\left(L_{3}\right)$ (resp. $\left(L_{1}\right)$ ), $\Sigma$ (resp. $g$ ) is well defined on $B_{\Sigma}$ (resp. $B_{g}$ ). Using $\left(L_{3}\right)$ and $\left(L_{2}\right)$, Theorem 2.1 ensures that $\Phi$ is well defined on $B_{\Phi}$, $\Phi\left(B_{\Phi}\right) \subseteq B_{\Sigma}$ and

$$
\left[\Phi-\left(x_{0}, y_{0}\right)\right]_{\left(z_{0}, y_{0}\right)} \leq \frac{\lambda_{\Phi} t}{1-\rho_{\Phi} t} .
$$

Composing this series majoration with that of $\left(L_{1}\right)$, Corollary 1.9 applied with $h=g \circ \Phi$ gives $\left[h-h\left(z_{0}, y_{0}\right)\right]_{\left(z_{0}, y_{0}\right)} \leq \lambda t /(1-\rho t)$ and $\Phi\left(B_{h}\right) \subseteq B_{\Sigma} \cap B_{g}$. From their definitions, observe that $B_{h} \subseteq B_{\Phi}$. It follows that $h$ is well defined on $B_{h}$.

From the definition of $z_{0}$, one has $\left\|z_{0}\right\|=\beta\left(\Sigma-\left(0, y_{0}\right) ; x_{0}, y_{0}\right) \leq \beta_{x}$ (from $\left(L_{3}\right)$ ), hence $\left(L_{4}\right)$ implies $\left(0, y_{0}\right) \in B_{h}$. We deduce that $\phi(y)$ is well defined on $B_{\phi}$ and Part (d) of Theorem 2.1 implies

$$
\left\{(x, y) \in B_{\Sigma} \mid y \in B_{\phi}, f(x, y)=0\right\}=\left\{(\phi(y), y) \mid y \in B_{\phi}\right\},
$$

which, combined to Lemma 3.5, leads to:

$$
\begin{array}{r}
\left\{(x, y) \in B_{\Sigma} \cap B_{g} \mid y \in B_{\phi}, f(x, y)=g^{[l]}(x, y)=0\right\} \\
=\left\{(\phi(y), y) \mid y \in B_{\phi}, D_{y}^{l} h(0, y)=0\right\} .
\end{array}
$$

From $\left(L_{2}\right)$ and the definition of $\Sigma$, we have $\sigma_{x} \geq 1$ and therefore

$$
\gamma_{x} \leq \sigma_{x} \gamma_{x}=(3-2 \sqrt{2}) \rho_{\Phi} \leq(3-2 \sqrt{2}) \rho .
$$

Successively using $\left(L_{3}\right)$ and $\left(L_{4}\right)$ we deduce:

$$
\beta\left(\Sigma-\left(0, y_{0}\right) ; x_{0}, y_{0}\right) \gamma\left(\Sigma ; x_{0}, y_{0}\right) \leq \alpha_{x}:=\beta_{x} \gamma_{x} \leq(3-2 \sqrt{2}) \rho \beta_{x}<3-2 \sqrt{2} .
$$

hence Corollary 1.18 gives:

$$
\left\|z_{0}^{\prime}\right\|<q\left(\alpha_{x}\right)^{2^{\kappa}-1} \beta_{x}
$$

where the function $q$ is defined in (15). Since $q\left(\alpha_{x}\right)<1$ and by the definition of $\kappa$, we deduce

$$
\left\|z_{0}^{\prime}\right\|<q\left(\alpha_{x}\right)^{m-l-1} \beta_{x} \leq \beta_{x} .
$$

Although we referred to this inequality just before the proof, as a motivation of the definition of $\kappa$, we will not use it in the remainder of the proof.

Using the inequalities $\left\|z_{0}^{\prime}-z_{0}\right\| \leq \beta_{x}+\left\|z_{0}^{\prime}\right\|$ and $\left(L_{4}\right)$, Corollary 1.6 on majorant series translation leads to:

$$
\left[h-h\left(z_{0}^{\prime}, y_{0}\right)\right]_{\left(z_{0}^{\prime}, y_{0}\right)} \leq \frac{\bar{\lambda} t}{1-\bar{\rho} t} .
$$

We are now ready to deduce point estimates of $h(0,$.$) from h\left(z_{0}^{\prime},.\right)$ at $y_{0}$. Using $\left(L_{5}\right),\left(L_{6}\right)$ and $\left(L_{7}\right)$, Proposition 3.6 yields: $D_{y}^{m} h\left(0, y_{0}\right) \neq 0$ and

$$
\beta_{m, l}\left(h(0, .) ; y_{0}\right) \leq \bar{\beta}_{m, l}, \quad \gamma_{m}\left(h(0, .) ; y_{0}\right) \leq \bar{\gamma}_{m} .
$$

Using $\left(L_{8}\right)$, we now proceed to zero location, via Theorem 3.1: $D_{y}^{l} h(0, y)$ admits $m-l$ zeroes $Z_{h}$ in $\bar{B}\left(y_{0}, r_{y}^{-}\right)$and in $\bar{B}\left(y_{0}, r_{y}^{+}\right)$. Remark that $\bar{\gamma}_{m} \geq$ $\bar{\mu} \geq \bar{\rho} \geq \rho /\left(1-\beta_{x} \rho\right)$, from which follows:

$$
\beta_{x}+r_{y}^{-} \leq \beta_{x}+r_{y}^{+}<\beta_{x}+\frac{1}{\bar{\gamma}_{m}} \leq \frac{1}{\rho} .
$$


We deduce $Z_{h} \subseteq \bar{B}\left(y_{0}, r_{y}^{-}\right) \subseteq \bar{B}\left(y_{0}, r_{y}^{+}\right) \subseteq B_{\phi}$. From (26), we deduce, for any $r_{y} \in\left\{r_{y}^{-}, r_{y}^{+}\right\}$:

$$
\begin{aligned}
& \left\{(x, y) \in B_{\Sigma} \cap B_{g} \mid y \in \bar{B}\left(y_{0}, r_{y}\right), f(x, y)=g^{[l]}(x, y)=0\right\} \\
& \quad=\left\{(\phi(y), y) \mid y \in \bar{B}\left(y_{0}, r_{y}\right), D_{y}^{l} h(0, y)=0\right\} \\
& \quad=\left\{(\phi(y), y) \mid y \in Z_{h}\right\} .
\end{aligned}
$$

For $r_{y}=r_{y}^{+}$, this gives the second half of the conclusion. As for the first half, from (29) and the evaluation of (24), via Proposition 1.4, for any $\zeta_{y} \in Z_{h}$ we have

$$
\left\|\Phi\left(0, \zeta_{y}\right)-\Phi\left(z_{0}, y_{0}\right)\right\| \leq r_{x}^{-},
$$

which concludes the proof.

\section{Cluster Approximation}

In this section, we present an approximation algorithm for clusters of embedding dimension 1, with the same features as the one given in [12] for univariate functions: either quadratic convergence holds or the current iterate lies at a distance of the cluster which is about its diameter. More generally, we shall parameterize our operator by $l \in\{0, \ldots, m-1\}$ and $l^{\prime} \leq l$ in order to approximate clusters of $\left(f, g^{\left[l^{\prime}\right]}\right)$ using the univariate Schröder operator on $D_{y}^{l} h$ (with respect to $y$ ).

We carry on with our main notation, $f: U_{f} \rightarrow \mathbb{C}^{n-1}$ and $g: U_{g} \rightarrow \mathbb{C}$ are analytic maps defined on maximal analyticity domains. We assume that $U:=U_{f} \cap U_{g}$ is not empty and for short we let $h(z, y):=h\left(f, g, x_{0}, y_{0} ; x, y\right)$, $\Sigma(x, y):=\Sigma\left(f, x_{0}, y_{0} ; x, y\right), \Phi(z, y):=\Phi\left(f, x_{0}, y_{0} ; z, y\right)$ and $(\phi(y), y):=$ $\Phi(0, y)$, where $\left(x_{0}, y_{0}\right) \in U$ denotes the initial point of the iteration. The functions $\mathcal{B}_{m, l^{\prime}}, \tau_{m, l, 0}$ and $\tau_{m, l, 1}$ are the ones introduced in Section 3.2.

We use the following quantities, that come from the univariate situation [12]:

$$
\begin{aligned}
\theta_{m, l, \delta} & :=\delta \frac{1}{m}+\frac{m+1}{(m-l+1)(m-l)}, \\
u_{m, l, \delta} & :=\max \left(u \geq 0 \mid u<1-(1 / 2)^{1 /(l+2)} \text { and } \frac{\theta_{m, l, \delta} u}{\psi_{l+1}(u)} \leq 1\right), \\
C_{m, l, l^{\prime}, \delta}(u) & :=\frac{1-u}{\psi_{m}(u)} \frac{(1-u)^{\frac{l^{\prime}+1}{m-l^{\prime}}}+\frac{\theta_{m, l, \delta}(2 m-1)}{\psi_{l+1}(u)}}{\left(1-\frac{\theta_{m, l, \delta} u}{\psi_{l+1}(u)}\right)^{2}} .
\end{aligned}
$$

5.1. Algorithm. The approximation algorithm depends on the initial point $\left(x_{0}, y_{0}\right)$ and on three positive real numbers $r_{y}, \mathcal{G}_{y}$ and $\mathcal{G}_{z}$ that will be assigned later.

An iteration of the algorithm computes $\left(x_{k+1}, y_{k+1}\right)$ from $\left(x_{k}, y_{k}\right)$. A rough description is as follows: first, Newton iteration on $f\left(., y_{k}\right)$ is applied a certain number of times with starting point $x_{k}$ to compute a new value $x_{k}^{\prime}$; Then we compute $z_{k}^{\prime}$ as the $n-1$ first coordinates of $\Sigma\left(x_{k}^{\prime}, y_{k}\right)$ and the Schröder operator is applied on $D_{y}^{l} h\left(z_{k},.\right)$ with starting point $y_{k}$, this gives a value $y_{k}^{\prime}$; then a discussion takes place to determine which of $y_{k}$ and $y_{k}^{\prime}$ 
should be taken for $y_{k+1}$; finally, $y_{k+1}$ is used in one Newton iteration on $f\left(., y_{k+1}\right)$ with starting point $x_{k}$ to compute $x_{k+1}$.

More formally, we introduce the operator $N_{m, l, l^{\prime}}(x, y)$, defined by the following algorithm, in which $\kappa$ represents the smallest integer such that $2^{\kappa} \geq 2\left(m-l^{\prime}\right)$. We also introduce the flag $\mathcal{F}_{m, l, l^{\prime}}^{y}\left(x_{k}, y_{k}\right)$ with values in the set of symbols $\{\infty,+,-, 1\}$, that keeps track of the branchings.

$\left(x_{k+1}, y_{k+1}\right):=N_{m, l, l^{\prime}}\left(x_{k}, y_{k}\right)$ is defined by

(1) $x_{k}^{\prime}:=N^{\kappa}\left(f\left(., y_{k}\right) ; x_{k}\right)$;

(2) $z_{k}^{\prime}:=D_{x} f\left(x_{0}, y_{0}\right)^{-1} f\left(x_{k}^{\prime}, y_{k}\right)$;

(3) if $D_{y}^{l+1} h\left(z_{k}^{\prime}, y_{k}\right)=0$

(4) then

(5)

$y_{k+1}:=y_{k}$

$\mathcal{F}_{m, l, l^{\prime}}^{y}\left(x_{k}, y_{k}\right):=\infty$

(6) else

(21) $x_{k+1}:=N\left(f\left(., y_{k+1}\right) ; x_{k}\right)$;

$y_{k}^{\prime}:=N_{m-l}\left(D_{y}^{l} h\left(z_{k}^{\prime},.\right) ; y_{k}\right) ;$

if $y_{k}^{\prime} \notin \bar{B}\left(y_{k}, 2 r_{y}\right)$

then

In a similar way, we introduce $\mathcal{F}_{m, l, l^{\prime}}^{z}\left(x_{k}, y_{k}\right)$ that takes the value 1 if

$$
\mathcal{B}_{m, l^{\prime}}\left(h\left(z_{k}^{\prime}, .\right), y_{k} ; y_{k+1}\right) \leq \mathcal{G}_{z}\left\|z_{k}^{\prime}\right\|^{1 /\left(m-l^{\prime}\right)}
$$

and 0 otherwise. These flags are to be used for stopping the iteration.

Informally speaking, $x_{k}^{\prime}$ is obtained from Newton's iteration in order to get $\left\|z_{k}^{\prime}\right\|^{1 /\left(m-l^{\prime}\right)}$ of the second order, that is in $\mathcal{O}\left(\left\|\left(x_{k}, y_{k}\right)-\zeta\right\|^{2}\right)$. Then we apply Schröder's operator to $D_{y}^{l} h\left(z_{k}^{\prime},.\right)$ at $y_{k}$. This is where we deeply use [12, Section 4] and different cases happen. Step (10) corresponds to $y_{k}^{\prime}$ getting far from $y_{k}$, which implies that $y_{k}$ is close to the cluster of $D_{y}^{l} h\left(z_{k}^{\prime},.\right)$. On the other hand, Step (20) corresponds to the fact that $y_{k}^{\prime}$ is closer to this cluster at the second order. In the meanwhile, the test of Step (14) determines the one among $y_{k}$ and $y_{k}^{\prime}$ which is closer to this cluster. At the end, the correction between $y_{k+1}$ and $y_{k}$ is propagated to the $x$ coordinates at Step (21).

The convergence analysis of the sequence $\left(x_{k}, y_{k}\right)_{k \in \mathbb{N}}$ is presented in the following theorem. The way of turning it into a practical algorithm is the goal of the next section. 
Theorem 5.1. Let $\zeta:=\left(\zeta_{x}, \zeta_{y}\right) \in U$ be such that $f(\zeta)=0$ and $D_{x} f(\zeta)$ is invertible. Let $\left(x_{0}, y_{0}\right) \in \bar{B}(\zeta, r)$, for a given $r \geq 0$. Let $m$ be such that $D_{y}^{m} h\left(0, \zeta_{y}\right) \neq 0, l \in\{0, \ldots, m-1\}$ and $l^{\prime} \leq l$. Let $r_{y}, \gamma_{x}, \sigma_{x}, \lambda_{g}, \rho_{g}, \bar{\gamma}_{x}$, $\bar{\delta}_{x}, \bar{\beta}_{m, l^{\prime}}, \bar{\gamma}_{m}, \bar{\sigma}_{m}$ be given and let:

$$
\begin{aligned}
& \delta:=1 \\
& \lambda_{\Phi}:=\sigma_{x} ; \rho_{\Phi}:=(3+2 \sqrt{2}) \sigma_{x} \gamma_{x} ; \\
& \lambda:=\lambda_{g} \lambda_{\Phi} ; \rho:=\rho_{\Phi}+\lambda_{\Phi} \rho_{g} \\
& l_{\phi}:=\frac{\lambda_{\Phi}}{1-\rho_{\Phi} r} ; L_{\phi}:=1+l_{\phi} ; r_{x}:=2 L_{\phi} r ; u_{x}:=\bar{\gamma}_{x} r_{x} \text {; } \\
& L_{z}:=L_{\phi}^{2}\left(\frac{\bar{\gamma}_{x}}{\psi\left(u_{x}\right)}\right)^{2-1 /\left(m-l^{\prime}\right)}\left(\frac{\bar{\delta}_{x}}{1-u_{x}}\right)^{1 /\left(m-l^{\prime}\right)} ; r_{z}:=\left(L_{z} r^{2}\right)^{m-l^{\prime}} ; \\
& \bar{r}:=4 r_{y} ; \\
& \bar{\lambda}:=\frac{\lambda}{(1-\rho \bar{r})^{2}} ; \bar{\rho}:=\frac{\rho}{1-\rho \bar{r}} ; \\
& \bar{\mu}:=\frac{\bar{\rho}}{1-\bar{\rho} r_{z}} ; L_{\bar{e}}:=\left((m+1)\left(\frac{m}{m+1}\right)^{m-l^{\prime}} \bar{\mu}^{l^{\prime}} \bar{\sigma}_{m} \frac{\bar{\lambda}}{1-\bar{\rho} r_{z}}\right)^{1 / m} ; \\
& \bar{e}:=\left((m+1) \bar{\sigma}_{m} \frac{\bar{\lambda} r_{z}}{1-\bar{\rho} r_{z}}\right)^{1 / m} ; \\
& u:=\bar{\gamma}_{m} r_{y} ; v:=2 u ; \bar{u}:=3 u ; \\
& \mathcal{C}:=\tau_{m, l^{\prime}, 1}(v) C_{m, l, l^{\prime}, \delta}(u)+\tau_{m, l^{\prime}, 0}(v) \text {; } \\
& \mathcal{G}_{y}:=\mathcal{C} \bar{\gamma}_{m} \\
& \overline{\mathcal{C}}:=\tau_{m, l^{\prime}, 1}(v) \mathcal{C}+\tau_{m, l^{\prime}, 0}(v) \text {; } \\
& \underline{\kappa}:=\frac{\tau_{m, l^{\prime}, 1}(v)}{1-\frac{\tau_{m, l^{\prime}, 0}(v)}{\mathcal{C}}} ; \\
& \bar{\kappa}:=\tau_{m, l^{\prime}, 1}(v)+\frac{\tau_{m, l^{\prime}, 0}(v)}{\mathcal{C}} ; \\
& \chi:=\frac{1-\bar{u}}{\psi_{m}(\bar{u})}\left((1-\bar{u})^{\frac{l^{\prime}+1}{m-l^{\prime}}}+\frac{2 m-1}{u_{m, l, \delta}}\right) ; \\
& \Xi:=\underline{\kappa} \chi \\
& T_{1}:=\frac{1}{1-(\bar{\mu} \bar{e})^{m}} \frac{1-u}{\psi_{m}(u)}\left((1-u)^{\frac{l^{\prime}+1}{m-l^{\prime}}}+\frac{3 \frac{m-l^{\prime}}{m}(2 m-1)}{1-(\bar{\mu} \bar{e})^{m}}\right) ; \\
& T_{2}:=\frac{L_{\bar{e}}^{m /\left(m-l^{\prime}\right)}}{1-(\bar{\mu} \bar{e})^{m}}\left(1+\frac{1}{1-(\bar{\mu} \bar{e})^{m}} \frac{3 \frac{m-l^{\prime}}{m}(2 m-1)(1-u)}{\psi_{m}(u)}\right) ; \\
& T_{\beta}:=2 \Xi T_{1} \text {; } \\
& \mathcal{G}_{z}:=2 \Xi T_{2} \text {; } \\
& T_{y}:=3 \frac{m-l^{\prime}}{m}\left(\left(1+(\bar{\mu} \bar{e})^{m}\right) \bar{\kappa}+L_{\bar{e}}^{m /\left(m-l^{\prime}\right)} / \mathcal{G}_{z}\right) ;
\end{aligned}
$$




$$
\begin{aligned}
& L_{y, 1}:= 3 \frac{m-l^{\prime}}{m}\left[\left(1+(\bar{\mu} \bar{e})^{m}\right) \overline{\mathcal{C}} \bar{\gamma}_{m}\left(1+3 \frac{m-l^{\prime}}{m} L_{\bar{e}}^{m /\left(m-l^{\prime}\right)} L_{z} r\right)^{2}\right. \\
&\left.\left(1-3 \frac{m-l^{\prime}}{m}\left(1+(\bar{\mu} \bar{e})^{m}\right) \overline{\mathcal{C}} v\right)^{-2}+L_{\bar{e}}^{m /\left(m-l^{\prime}\right)} L_{z}\right] \\
& L_{y, 2}:= 3 \frac{m-l^{\prime}}{m}\left(\left(1+(\bar{\mu} \bar{e})^{m}\right) \bar{\kappa} \mathcal{G}_{z}+L_{\bar{e}}^{m /\left(m-l^{\prime}\right)}\right) L_{z} ; \\
& L_{y}:=\max \left(L_{y, 1}, L_{y, 2}\right) ; L_{x}:=\frac{4 \bar{\gamma}_{x}}{\psi\left(u_{x}\right)} L_{\phi}^{2}+L_{\phi} L_{y} .
\end{aligned}
$$

Assume:

$\left(A_{1}\right) \gamma_{x} \geq \gamma(\Sigma ; \zeta) ; \sigma_{x} \geq\left\|D \Sigma(\zeta)^{-1}\right\| ;$

$\left(A_{2}\right)[g-g(\zeta)]_{\zeta} \leq \frac{\lambda_{g} t}{1-\rho_{g} t}$

$\left(A_{3}\right) \rho_{\phi} r<1$;

$\left(A_{4}\right) \bar{\gamma}_{x} \geq \max \left(\gamma(\Sigma ; \phi(y), y) \mid y \in \bar{B}\left(\zeta_{y}, r\right)\right)$;

$\left(A_{5}\right) \bar{\delta}_{x} \geq \max \left(\|D \Sigma(\phi(y), y)\| \mid y \in \bar{B}\left(\zeta_{y}, r\right)\right)$;

(A6) $u_{x}<\frac{5-\sqrt{17}}{4}$;

$\left(A_{7}\right) r_{y} \geq r$

$\left(A_{8}\right) r_{y} \geq 3 \frac{m-l^{\prime}}{m} \max \left(\beta_{m, l^{\prime}}(h(z,) ; y) \mid. z \in \bar{B}\left(0, r_{z}\right), y \in \bar{B}\left(\zeta_{y}, r\right)\right) ;$

$\left(A_{9}\right) \rho\left(r_{z}+\bar{r}\right)<1$

$\left(A_{10}\right)+\infty>\bar{\sigma}_{m} \geq \max \left(m ! /\left|D_{y}^{m} h(0, y)\right| \mid y \in \bar{B}\left(\zeta_{y}, \bar{r}\right)\right)$;

$\left(A_{11}\right) \bar{\beta}_{m, l^{\prime}} \geq \max \left(\beta_{m, l^{\prime}}(h(z,) ; y) \mid. z \in \bar{B}\left(0, r_{z}\right), y \in \bar{B}\left(\zeta_{y}, \bar{r}\right)\right)$;

$\left(A_{12}\right) \bar{\gamma}_{m} \geq \max \left(\gamma_{m}(h(z,) ; y) \mid. z \in \bar{B}\left(0, r_{z}\right), y \in \bar{B}\left(\zeta_{y}, \bar{r}\right)\right)$;

$\left(A_{13}\right) \bar{\rho} r_{z}<1 ; \bar{\mu} \bar{e}<1$;

(A14) $\frac{m-l^{\prime}}{m} \frac{m+1}{m+1-l^{\prime}} \bar{\beta}_{m, l^{\prime}} \bar{\gamma}_{m} \leq 1 / 9$;

$\left(A_{15}\right) r_{y} \frac{m+1}{m+1-l^{\prime}} \bar{\gamma}_{m} \leq 1 / 12 ;$

$\left(A_{16}\right) u<u_{m, l, \delta} ; \bar{u}<1-(1 / 2)^{1 /(m+1)} ; v<v_{m, l^{\prime}} ;$

(A $\left.A_{17}\right) 3 \frac{m-l^{\prime}}{m}\left(1+(\bar{\mu} \bar{e})^{m}\right) \overline{\mathcal{C}} v<1$;

$\left(A_{18}\right) L_{y} r<1$

$\left(A_{19}\right) L_{x} r<1$.

Then, there exists a cluster $\mathcal{Z}_{0, \zeta_{y}}$ of $m-l^{\prime}$ zeroes of $D_{y}^{l^{\prime}} h(0,$.$) in \bar{B}\left(\zeta_{y}, r_{y}\right)$, counting multiplicities. Assume that $\zeta_{y}$ belongs to the convex hull of $\mathcal{Z}_{0, \zeta_{y}}$. Let $\left(x_{k}, y_{k}\right)_{k \geq 0}$ be the sequence formally defined by induction according to:

$$
\left(x_{k+1}, y_{k+1}\right):=N_{m, l, l^{\prime}}\left(x_{k}, y_{k}\right) .
$$

Let $K$ be the first integer such that

$$
\mathcal{F}_{m, l, l^{\prime}}^{y}\left(x_{K}, y_{K}\right) \neq 1 \text { and } \mathcal{F}_{m, l, l^{\prime}}^{z}\left(x_{K}, y_{K}\right)=0,
$$


or $+\infty$ if no such integer exists. Then, for all $k \leq K,\left(x_{k}, y_{k}\right)$ is well defined.

a. For all $k<K$ we have:

$$
\left\|\left(x_{k+1}, y_{k+1}\right)-\zeta\right\| \leq L_{x}\left\|\left(x_{k}, y_{k}\right)-\zeta\right\|^{2} \leq r .
$$

b. If $K$ is finite then $y_{K+1}$ is well defined and satisfies:

$$
\left|y_{K+1}-\zeta_{y}\right| \leq T_{y} \mathcal{B}_{m, l^{\prime}}\left(h\left(z_{K}^{\prime}, .\right), y_{K} ; y_{K+1}\right)
$$

and

$$
\mathcal{B}_{m, l^{\prime}}\left(h\left(z_{K}^{\prime}, .\right), y_{K} ; y_{K+1}\right) \leq T_{\beta} \beta_{m, l^{\prime}}\left(h(0, .) ; \zeta_{y}\right) .
$$

For short, we write $\beta_{m, l^{\prime}}:=\beta_{m, l^{\prime}}\left(h(0,.) ; \zeta_{y}\right)$. The rest of this section is devoted to the proof of this theorem. The notation is the same as in the theorem. We proceed by induction on $k$ : we assume that $\left(\left(x_{j}, y_{j}\right)\right)_{0 \leq j \leq k}$ is well defined up to a certain index $k \leq K$ and that all its elements belong to $\bar{B}(\zeta, r)$. The proof of Part (b) is addressed at the end of the section.

5.2. Definition Domains. We first provide definition domains for all the maps involved in the algorithm. We introduce:

$$
\begin{gathered}
B_{\Sigma}:=B\left(\zeta, \frac{1-\sqrt{2} / 2}{\gamma_{x}}\right), B_{g}:=B\left(\zeta, \frac{1}{\rho_{g}}\right), \\
B_{\Phi}:=B\left(\left(0, \zeta_{y}\right), \frac{1}{\rho_{\Phi}}\right), B_{h}:=B\left(\left(0, \zeta_{y}\right), \frac{1}{\rho}\right) .
\end{gathered}
$$

Lemma 5.2. $\Sigma$ (resp. $\Phi)$ is well defined on $B_{\Sigma}$ (resp. $\left.B_{\Phi}\right) . h$ is well defined as the composition $g \circ \Phi$ on $B_{h}$.

Proof. Using $\left(A_{1}\right)$ and $\left(A_{2}\right)$ we have $B_{\Sigma} \subseteq U_{f}, B_{g} \subseteq U_{g}$ and Theorem 2.1 about local inversion ensures that $\Phi$ is well defined on $B_{\Phi}, \Phi\left(B_{\Phi}\right) \subseteq B_{\Sigma}$ and

$$
[\Phi-\zeta]_{\left(0, \zeta_{y}\right)} \leq \frac{\lambda_{\Phi} t}{1-\rho_{\Phi} t}
$$

Then, according to Corollary 1.9 on majorant series composition applied to $h=g \circ \Phi$, we get

$$
\left[h-h\left(0, \zeta_{y}\right)\right]_{\left(0, \zeta_{y}\right)} \leq \frac{\lambda t}{1-\rho t}
$$

and $\Phi\left(B_{h}\right) \subseteq B_{g}$, which means that $h=g \circ \Phi$ is well defined on $B_{h}$.

5.3. Uniform Convergence to the Curve. According to our hypotheses, $f(x, y)=0$ defines a smooth curve in a neighborhood of $\zeta$. We perform a uniform convergence analysis to this curve for the operator used in Step (1) of the algorithm. We start with two lemmas.

Lemma 5.3. For all $(a, b) \in \bar{B}\left(\zeta_{x}, r\right) \times \bar{B}\left(\zeta_{y}, r\right)$ we have

$$
\begin{aligned}
\|(\phi(b), b)-\zeta\| & \leq l_{\phi}\left|b-\zeta_{y}\right|, \\
\|a-\phi(b)\| & \leq L_{\phi}\|(a, b)-\zeta\| .
\end{aligned}
$$

Proof. Using $\left(A_{3}\right)$, the evaluation of (32) by means of Proposition 1.4 yields:

$$
\|(\phi(b), b)-\zeta\|=\left\|\Phi(0, b)-\Phi\left(0, \zeta_{y}\right)\right\| \leq l_{\phi}\left|b-\zeta_{y}\right|,
$$

which implies

$$
\|a-\phi(b)\| \leq\left\|a-\zeta_{x}\right\|+\left\|\phi(b)-\zeta_{x}\right\| \leq L_{\phi}\|(a, b)-\zeta\| .
$$


Lemma 5.4. For any $(a, b) \in \bar{B}\left(\zeta_{x}, r\right) \times \bar{B}\left(\zeta_{y}, r\right)$, and any integers $j \geq 0$ and $p \leq 2^{j}, a^{\prime}:=N^{j}(f(., b), a)$ is well defined and

$$
\left\|a^{\prime}-\phi(b)\right\| \leq\left(\frac{\bar{\gamma}_{x}\|a-\phi(b)\|}{\psi\left(u_{x}\right)}\right)^{p-1}\|a-\phi(b)\| \leq r_{x} .
$$

Proof. By the previous lemma, $\|a-\phi(b)\| \leq 2 L_{\phi} r=r_{x}$ holds. Then, using $\left(A_{4}\right)$, we deduce $\gamma(\Sigma ; \phi(b), b)\|a-\phi(b)\| \leq u_{x}$, which, via $\left(A_{6}\right)$, implies $\frac{u_{x}}{\psi\left(u_{x}\right)}<1$. Thus Theorem 1.16 gives:

$$
\left\|a^{\prime}-\phi(b)\right\| \leq\left(\frac{\bar{\gamma}_{x}\|a-\phi(b)\|}{\psi\left(u_{x}\right)}\right)^{2^{j}-1}\|a-\phi(b)\| \leq r_{x} .
$$

Using $\frac{u_{x}}{\psi\left(u_{x}\right)}<1$ yields the claimed bound.

We are now able to deduce that $z_{k}^{\prime} \in \bar{B}\left(0, r_{z}\right)$, which will be used several times in the remainder of the proof, without explicit reference:

Corollary 5.5. $\left\|z_{k}^{\prime}\right\| \leq L_{z}^{m-l^{\prime}}\left\|\left(x_{k}, y_{k}\right)-\zeta\right\|^{2\left(m-l^{\prime}\right)} \leq r_{z}$.

Proof. We apply the previous lemma to $(a, b):=\left(x_{k}, y_{k}\right), j:=\kappa$ and $p:=$ $2\left(m-l^{\prime}\right)$ :

$$
\left\|x_{k}^{\prime}-\phi\left(y_{k}\right)\right\| \leq\left(\frac{\bar{\gamma}_{x}\left\|x_{k}-\phi\left(y_{k}\right)\right\|}{\psi\left(u_{x}\right)}\right)^{2\left(m-l^{\prime}\right)-1}\left\|x_{k}-\phi\left(y_{k}\right)\right\| .
$$

By means of (35) (instanciated at $\left.(a, b):=\left(x_{k}, y_{k}\right)\right)$, we deduce:

$$
\left\|x_{k}^{\prime}-\phi\left(y_{k}\right)\right\| \leq\left(\frac{\bar{\gamma}_{x}}{\psi\left(u_{x}\right)}\right)^{2\left(m-l^{\prime}\right)-1} L_{\phi}^{2\left(m-l^{\prime}\right)}\left\|\left(x_{k}, y_{k}\right)-\zeta\right\|^{2\left(m-l^{\prime}\right)} .
$$

Using $\left(A_{4}\right)$ and $\left(A_{5}\right)$, we deduce the following series majoration:

$$
\left[\Sigma-\left(0, y_{k}\right)\right]_{\Phi\left(0, y_{k}\right)} \leq \frac{\bar{\delta}_{x} t}{1-\bar{\gamma}_{x} t}
$$

which evaluates at $\left(x_{k}^{\prime}, y_{k}\right)$ since $\bar{\gamma}_{x}\left\|x_{k}^{\prime}-\phi\left(y_{k}\right)\right\| \leq u_{x}<1$ (from $\left(A_{6}\right)$ ), by means of Proposition 1.4:

$$
\begin{aligned}
\left\|z_{k}^{\prime}\right\| & =\left\|\Sigma\left(x_{k}^{\prime}, y_{k}\right)-\left(0, y_{k}\right)\right\| \\
& \leq \frac{\bar{\delta}_{x}\left\|x_{k}^{\prime}-\phi\left(y_{k}\right)\right\|}{1-u_{x}} \leq L_{z}^{m-l^{\prime}}\left\|\left(x_{k}, y_{k}\right)-\zeta\right\|^{2\left(m-l^{\prime}\right)} \leq r_{z} .
\end{aligned}
$$

5.4. Uniform $z$-translation. From the previous result, $\left\|z_{k}^{\prime}\right\|^{1 /\left(m-l^{\prime}\right)}$ belongs to $\mathcal{O}\left(\left\|\left(x_{k}, y_{k}\right)-\zeta\right\|^{2}\right)$. We are now ready to apply our bound of Section 3.4 on $z$-translation in a uniform way with respect to $y$. We start with a uniform series majoration of $h$. First of all, it is important to notice that $\left(A_{9}\right)$ implies:

$$
B_{h} \supseteq \bar{B}\left(0, r_{z}\right) \times \bar{B}\left(\zeta_{y}, \bar{r}\right) .
$$

Lemma 5.6. For all $b \in \bar{B}\left(\zeta_{y}, \bar{r}\right)$, we have: $[h-h(0, b)]_{(0, b)} \leq \frac{\bar{\lambda} t}{1-\bar{\rho} t}$.

Proof. This directly follows from Corollary 1.6, using $\left(A_{9}\right)$ and (33). 
Lemma 5.7. For all $(c, b) \in \bar{B}\left(0, r_{z}\right) \times \bar{B}\left(\zeta_{y}, \bar{r}\right)$, we have $D_{y}^{m} h(c, b) \neq 0$ and

$$
\begin{aligned}
& \beta_{m, l^{\prime}}(h(0, .) ; b) \leq\left(1+(\bar{\mu} \bar{e})^{m}\right) \beta_{m, l^{\prime}}(h(c, .) ; b)+L_{\bar{e}}^{m /\left(m-l^{\prime}\right)}\|c\|^{1 /\left(m-l^{\prime}\right)}, \\
& \beta_{m, l^{\prime}}(h(c, .) ; b) \leq \frac{\beta_{m, l^{\prime}}(h(0, .) ; b)+L_{\bar{e}}^{m /\left(m-l^{\prime}\right)}\|c\|^{1 /\left(m-l^{\prime}\right)}}{1-(\bar{\mu} \bar{e})^{m}} .
\end{aligned}
$$

Proof. Using $\left(A_{10}\right),\left(A_{13}\right)$ and Lemma 5.6, Proposition 3.6 (with $\left(z_{0}, y_{0}\right):=$ $(0, b)$ and $\left.z_{1}:=c\right)$ implies these bounds.

5.5. Uniform Cluster Location. Now, we show quantitative results about clusters of zeroes of $D_{y}^{l^{\prime}} h(c,$.$) when c$ varies.

Lemma 5.8. For any $(c, b) \in \bar{B}\left(0, r_{z}\right) \times \bar{B}\left(\zeta_{y}, \bar{r}\right)$, there exists a cluster $\mathcal{Z}_{c, b}$ of $m-l^{\prime}$ zeroes of the analytic extension of $D_{y}^{l^{\prime}} h(c,$.$) in$

$$
\bar{B}\left(b, 3 \frac{m-l^{\prime}}{m} \beta_{m, l^{\prime}}(h(c, .) ; b)\right) \text { and } \bar{B}\left(b, \frac{m+1-l^{\prime}}{3(m+1) \gamma_{m}(h(c, .) ; b)}\right) .
$$

In addition, if $b \in \bar{B}\left(\zeta_{y}, r\right)$ then

$$
\bar{B}\left(b, 3 \frac{m-l^{\prime}}{m} \beta_{m, l^{\prime}}(h(c, .) ; b)\right) \subseteq \bar{B}\left(b, r_{y}\right) .
$$

Proof. The location of $\mathcal{Z}_{c, b}$ directly follows from $\left(A_{11}\right),\left(A_{12}\right)$ and $\left(A_{14}\right)$ and Theorem 3.1. The last ball inclusion rephrases $\left(A_{8}\right)$.

Lemma 5.9. For any $(c, b) \in \bar{B}\left(0, r_{z}\right) \times \bar{B}\left(\zeta_{y}, r\right)$ and any $b^{\prime} \in \bar{B}\left(b, 3 r_{y}\right)$, we have $\mathcal{Z}_{c, b}=\mathcal{Z}_{c, b^{\prime}}$. In particular, for any $b^{\prime \prime}$ in the convex hull of $\mathcal{Z}_{c, b}$, we have:

$$
\left|b^{\prime \prime}-b^{\prime}\right| \leq 3 \frac{m-l^{\prime}}{m} \beta_{m, l^{\prime}}\left(h(c, .) ; b^{\prime}\right) .
$$

Proof. From $\left(A_{7}\right)$, observe that $b^{\prime} \in \bar{B}\left(\zeta_{y}, \bar{r}\right)$, thus applying the previous lemma at $\left(c, b^{\prime}\right)$ gives the existence of a cluster $\mathcal{Z}_{c, b^{\prime}}$ contained in the ball $\bar{B}\left(b^{\prime}, 3 \frac{m-l^{\prime}}{m} \beta_{m, l^{\prime}}\left(h(c,.) ; b^{\prime}\right)\right)$ and also in $\bar{B}\left(b^{\prime}, \frac{m+1-l^{\prime}}{3(m+1) \gamma_{m}\left(h(c, .) ; b^{\prime}\right)}\right)$. Then, for any $b^{\prime \prime} \in \mathcal{Z}_{c, b}$ one has:

$$
\begin{aligned}
\left|b^{\prime \prime}-b^{\prime}\right| & \leq\left|b^{\prime \prime}-b\right|+\left|b-b^{\prime}\right| \\
& \leq r_{y}+\left|b-b^{\prime}\right| \quad(\text { using the previous lemma }) \\
& \leq 4 r_{y} \\
& \leq \frac{m+1-l^{\prime}}{3(m+1) \bar{\gamma}_{m}} \quad\left(\operatorname{using}\left(A_{15}\right)\right) \\
& \leq \frac{m+1-l^{\prime}}{3(m+1) \gamma_{m}\left(h(c, .) ; b^{\prime}\right)} \quad\left(\text { using }\left(A_{12}\right)\right) .
\end{aligned}
$$

We deduce $\mathcal{Z}_{c, b}=\mathcal{Z}_{c, b^{\prime}}$.

We now specialize these lemmas to our situation. For this purpose, we first notice:

Lemma 5.10. $\left|y_{k+1}-\zeta_{y}\right| \leq 3 r_{y} \leq \bar{r}$.

Proof. By construction, we have $\left|y_{k}-y_{k+1}\right| \leq 2 r_{y}$. Since $\left(A_{7}\right)$ implies $\left|y_{k}-\zeta_{y}\right| \leq r \leq r_{y}$, we deduce: $\left|y_{k+1}-\zeta_{y}\right| \leq\left|y_{k}-y_{k+1}\right|+\left|y_{k}-\zeta_{y}\right| \leq 3 r_{y} \leq$ $\bar{r}$. 
This statement will be invoked several times in the remainder of the proof without explicit reference. Lemma 5.9 is only used to prove the next two corollaries. The first statement of Theorem 5.1 about the location of the cluster $\mathcal{Z}_{0, \zeta_{y}}$ around $\zeta_{y}$ is a consequence of Lemma 5.8. From now, we assume that $\zeta_{y}$ belongs to the convex hull of this cluster.

Corollary 5.11. There exist $m-l^{\prime}$ zeroes of $D_{y}^{l^{\prime}} h(0,$.$) in \bar{B}\left(\zeta_{y}, r_{y}\right)$ and

$$
\left|y_{k+1}-\zeta_{y}\right| \leq 3 \frac{m-l^{\prime}}{m} \beta_{m, l^{\prime}}\left(h(0, .) ; y_{k+1}\right) \text {. }
$$

Proof. The inequality directly follows from Lemma 5.9, applied with $(c, b):=$ $\left(0, \zeta_{y}\right), b^{\prime}:=y_{k+1}$ and $b^{\prime \prime}:=\zeta_{y}$ (thanks to Lemma 5.10).

The last corollary concerns the location of the cluster around $\left(z_{k}^{\prime}, y_{k}\right)$.

Corollary 5.12. There exist $m-l^{\prime}$ zeroes of $D_{y}^{l^{\prime}} h\left(z_{k}^{\prime},.\right)$ in $\bar{B}\left(y_{k}, r_{y}\right)$. We denote by $\zeta_{k}$ a point lying in the convex hull of this cluster. Then one has:

$$
\left|\zeta_{k}-\zeta_{y}\right| \leq 3 \frac{m-l^{\prime}}{m} \beta_{m, l^{\prime}}\left(h\left(z_{k}^{\prime}, .\right) ; \zeta_{y}\right) \leq r_{y} .
$$

Proof. The first part directly follows from Lemma 5.8 applied with $(c, b):=$ $\left(z_{k}^{\prime}, y_{k}\right)$. From $\left(A_{7}\right)$, one has $\left|y_{k}-\zeta_{y}\right| \leq r \leq r_{y}$, hence the first inequality of (39) follows from applying Lemma 5.9 with $(c, b):=\left(z_{k}^{\prime}, y_{k}\right), b^{\prime}:=\zeta_{y}$ and $b^{\prime \prime}:=\zeta_{k}$. The second inequality directly follows from $\left(A_{8}\right)$.

5.6. Uniform Cluster Approximation. Let $\zeta_{k}$ be the one defined in Corollary 5.12. Here we show that the approximation algorithm of $[12$, Section 4.4] applies to $D_{y}^{l} h\left(z_{k}^{\prime},.\right)$.

Lemma 5.13. The following alternative holds about $y_{k+1}$ :

a. If $\mathcal{F}_{m, l, l^{\prime}}^{y}\left(x_{k}, y_{k}\right) \neq 1$ then

$$
\begin{aligned}
\beta_{m, l^{\prime}}\left(h\left(z_{k}^{\prime}, .\right) ; y_{k+1}\right) & \leq \bar{\kappa} \mathcal{B}_{m, l^{\prime}}\left(h\left(z_{k}^{\prime}, .\right), y_{k} ; y_{k+1}\right), \\
\mathcal{B}_{m, l^{\prime}}\left(h\left(z_{k}^{\prime}, .\right), y_{k} ; y_{k+1}\right) & \leq \Xi \beta_{m, l^{\prime}}\left(h\left(z_{k}^{\prime}, .\right) ; \zeta_{k}\right) ;
\end{aligned}
$$

b. If $\mathcal{F}_{m, l, l^{\prime}}^{y}\left(x_{k}, y_{k}\right)=1$ then

$$
\beta_{m, l^{\prime}}\left(h\left(z_{k}^{\prime}, .\right) ; y_{k+1}\right) \leq \overline{\mathcal{C}} \bar{\gamma}_{m}\left|y_{k}-y_{k+1}\right|^{2} .
$$

Proof. It suffices to check that the conditions of [12, Theorem 4.5] are satisfied with the analytic extension of $h\left(z_{k}^{\prime},.\right)$ at $\zeta_{k}$ with $r_{y}$. Namely, making use of $\left|\zeta_{k}-\zeta_{y}\right| \leq r_{y}$ (Corollary 5.12), we check:

- The function $\mathcal{B}_{m, l^{\prime}}$ satisfies the required properties, thanks to Proposition 3.3 ;

- $D_{y}^{m} h\left(z_{k}^{\prime}, \zeta_{k}\right) \neq 0$ by Lemma 5.7 ;

- $\bar{\gamma}_{m} \geq \gamma_{m}\left(h\left(z_{k}^{\prime},.\right) ; \zeta_{k}\right)$, thanks to $\left(A_{12}\right)$;

- $\bar{\gamma}_{m} \geq \max \left(\gamma_{m}\left(h\left(z_{k}^{\prime},.\right) ; y\right) \mid y \in \bar{B}\left(\zeta_{k}, 3 r_{y}\right)\right)$, thanks to $\left(A_{12}\right)$ again;

- $u<u_{m, l, \delta}, \bar{u}<1-(1 / 2)^{1 /(m+1)}, v<v_{m, l^{\prime}}$, from $\left(A_{16}\right)$.

Part (b) is directly extracted from [12, Theorem 4.5, Part (c)]. As for Part (a), we distinguish two cases.

First, if $D_{y}^{l+1} h\left(z_{k}^{\prime}, y_{k}\right)=0$ or $D_{y}^{l+1} h\left(z_{k}^{\prime}, y_{k}\right) \neq 0$ and $y_{k}^{\prime} \notin \bar{B}\left(y_{k}, 2 r_{y}\right)$ then from [12, Theorem 4.5, Part (a)] one has:

$$
\beta_{m, l^{\prime}}\left(h\left(z_{k}^{\prime}, .\right) ; y_{k}\right) \leq \chi \beta_{m, l^{\prime}}\left(h\left(z_{k}^{\prime}, .\right) ; \zeta_{k}\right) .
$$


In the second case, $y_{k}^{\prime} \in \bar{B}\left(y_{k}, 2 r_{y}\right)$ but $\mathcal{B}_{m, l^{\prime}}\left(h\left(z_{k}^{\prime},.\right), y_{k} ; y_{k}^{\prime}\right)>\mathcal{G}_{y}\left|y_{k}-y_{k}^{\prime}\right|^{2}$, from [12, Theorem 4.5, Part (b)] one has

$$
\min \left(\beta_{m, l^{\prime}}\left(h\left(z_{k}^{\prime}, .\right) ; y_{k}\right), \beta_{m, l^{\prime}}\left(h\left(z_{k}^{\prime}, .\right) ; y_{k}^{\prime}\right)\right) \leq \chi \beta_{m, l^{\prime}}\left(h\left(z_{k}^{\prime}, .\right) ; \zeta_{k}\right)
$$

and

$$
\begin{aligned}
\beta_{m, l^{\prime}}\left(h\left(z_{k}^{\prime}, .\right) ; y_{k}^{\prime}\right) & \leq \bar{\kappa} \mathcal{B}_{m, l^{\prime}}\left(h\left(z_{k}^{\prime}, .\right), y_{k} ; y_{k}^{\prime}\right), \\
\mathcal{B}_{m, l^{\prime}}\left(h\left(z_{k}^{\prime}, .\right), y_{k} ; y_{k}^{\prime}\right) & \leq \underline{\kappa} \beta_{m, l^{\prime}}\left(h\left(z_{k}^{\prime}, .\right) ; y_{k}^{\prime}\right) .
\end{aligned}
$$

Since $\bar{\kappa} \geq 1$ and $\beta_{m, l^{\prime}}\left(h\left(z_{k}^{\prime},.\right) ; y_{k}\right)=\mathcal{B}_{m, l^{\prime}}\left(h\left(z_{k}^{\prime},.\right), y_{k} ; y_{k}\right)$, we deduce (40). From (43) and $\underline{\kappa} \geq 1$, we also obtain

$$
\min \left(\beta_{m, l^{\prime}}\left(h\left(z_{k}^{\prime}, .\right) ; y_{k}\right), \mathcal{B}_{m, l^{\prime}}\left(h\left(z_{k}^{\prime}, .\right), y_{k} ; y_{k}^{\prime}\right)\right) \leq \underline{\kappa} \chi \beta_{m, l^{\prime}}\left(h\left(z_{k}^{\prime}, .\right) ; \zeta_{k}\right),
$$

which yields (41) by definition of $y_{k+1}$.

5.7. Proof of Part (a) of Theorem 5.1. We distinguish two cases:

Case 1: In this case, we assume $\mathcal{F}_{m, l, l^{\prime}}^{y}\left(x_{k}, y_{k}\right)=1$. Combining Part (b) of Lemma 5.13 and Lemma 5.7 (applied with $(c, b):=\left(z_{k}^{\prime}, y_{k+1}\right)$ ), we deduce:

$$
\beta_{m, l^{\prime}}\left(h(0, .) ; y_{k+1}\right) \leq\left(1+(\bar{\mu} \bar{e})^{m}\right) \overline{\mathcal{C}} \bar{\gamma}_{m}\left|y_{k+1}-y_{k}\right|^{2}+L_{\bar{e}}^{m /\left(m-l^{\prime}\right)}\left\|z_{k}^{\prime}\right\|^{1 /\left(m-l^{\prime}\right)}
$$

hence Corollaries 5.5 and 5.11 imply

$$
\begin{aligned}
\left|y_{k+1}-\zeta_{y}\right| \leq 3 \frac{m-l^{\prime}}{m}( & \left(1+(\bar{\mu} \bar{e})^{m}\right) \overline{\mathcal{C}}_{m}\left|y_{k+1}-y_{k}\right|^{2} \\
& \left.+L_{\bar{e}}^{m /\left(m-l^{\prime}\right)} L_{z}\left\|\left(x_{k}, y_{k}\right)-\zeta\right\|^{2}\right) .
\end{aligned}
$$

Then, from $\left|y_{k}-y_{k+1}\right| \leq\left|y_{k}-\zeta_{y}\right|+\left|y_{k+1}-\zeta_{y}\right|$ we deduce:

$$
\begin{aligned}
\left|y_{k}-y_{k+1}\right| \leq\left|y_{k}-\zeta_{y}\right| & +3 \frac{m-l^{\prime}}{m}\left(\left(1+(\bar{\mu} \bar{e})^{m}\right) \overline{\mathcal{C}} v\left|y_{k+1}-y_{k}\right|\right. \\
& \left.+L_{\bar{e}}^{m /\left(m-l^{\prime}\right)} L_{z}\left\|\left(x_{k}, y_{k}\right)-\zeta\right\|^{2}\right) .
\end{aligned}
$$

Using $\left(A_{17}\right)$ yields

$$
\begin{aligned}
\left|y_{k}-y_{k+1}\right| & \leq \frac{\left|y_{k}-\zeta_{y}\right|+3 \frac{m-l^{\prime}}{m} L_{\bar{e}}^{m /\left(m-l^{\prime}\right)} L_{z}\left\|\left(x_{k}, y_{k}\right)-\zeta\right\|^{2}}{1-3 \frac{m-l^{\prime}}{m}\left(1+(\bar{\mu} \bar{e})^{m}\right) \overline{\mathcal{C}} v} \\
& \leq \frac{1+3 \frac{m-l^{\prime}}{m} L_{\bar{e}}^{m /\left(m-l^{\prime}\right)} L_{z} r}{1-3 \frac{m-l^{\prime}}{m}\left(1+(\bar{\mu} \bar{e})^{m}\right) \overline{\mathcal{C}} v}\left\|\left(x_{k}, y_{k}\right)-\zeta\right\| .
\end{aligned}
$$

Combining (44) and (45) leads to

$$
\left|y_{k+1}-\zeta_{y}\right| \leq L_{y, 1}\left\|\left(x_{k}, y_{k}\right)-\zeta\right\|^{2} .
$$

Case 2: Now we examine the case when

$$
\mathcal{F}_{m, l, l^{\prime}}^{y}\left(x_{k}, y_{k}\right) \neq 1 \text { but } \mathcal{F}_{m, l, l^{\prime}}^{z}\left(x_{k}, y_{k}\right)=1 .
$$

Successively using Lemma 5.7 (with $(c, b):=\left(z_{k}^{\prime}, y_{k+1}\right)$ ) and Part (a) of Lemma 5.13, we deduce

$$
\beta_{m, l^{\prime}}\left(h(0, .) ; y_{k+1}\right) \leq\left(\left(1+(\bar{\mu} \bar{e})^{m}\right) \bar{\kappa} \mathcal{G}_{z}+L_{\bar{e}}^{m /\left(m-l^{\prime}\right)}\right)\left\|z_{k}^{\prime}\right\|^{1\left(m-l^{\prime}\right)},
$$

which leads to, by means of Corollaries 5.11 and 5.5:

$$
\left|y_{k+1}-\zeta_{y}\right| \leq L_{y, 2}\left\|\left(x_{k}, y_{k}\right)-\zeta\right\|^{2} \text {. }
$$


This concludes this second case.

We are now ready to conclude the proof of Part (a) of Theorem 5.1. According to the definition of $L_{y}$ and $\left(A_{18}\right)$, bounds (46) and (47) imply:

$$
\left|y_{k+1}-\zeta_{y}\right| \leq L_{y}\left\|\left(x_{k}, y_{k}\right)-\zeta\right\|^{2} \leq\left\|\left(x_{k}, y_{k}\right)-\zeta\right\| \leq r .
$$

From Lemma 5.4 (applied with $\left.(a, b):=\left(x_{k}, y_{k+1}\right)\right)$, we have:

$$
\left\|x_{k+1}-\phi\left(y_{k+1}\right)\right\| \leq \frac{\bar{\gamma}_{x}}{\psi\left(u_{x}\right)}\left\|x_{k}-\phi\left(y_{k+1}\right)\right\|^{2} .
$$

Using Lemma 5.3, we also have

$$
\begin{aligned}
\left\|x_{k}-\phi\left(y_{k+1}\right)\right\| & \leq L_{\phi}\left\|\left(x_{k}, y_{k+1}\right)-\zeta\right\| \\
& \leq L_{\phi}\left(\left\|x_{k}-\zeta_{x}\right\|+\left|y_{k+1}-\zeta_{y}\right|\right) \\
& \leq 2 L_{\phi}\left\|\left(x_{k}, y_{k}\right)-\zeta\right\|,
\end{aligned}
$$

from which follows:

$$
\left\|x_{k+1}-\phi\left(y_{k+1}\right)\right\| \leq \frac{4 \bar{\gamma}_{x}}{\psi\left(u_{x}\right)} L_{\phi}^{2}\left\|\left(x_{k}, y_{k}\right)-\zeta\right\|^{2} .
$$

Lemma 5.3 then gives $\left\|\phi\left(y_{k+1}\right)-\zeta_{x}\right\| \leq l_{\phi}\left|y_{k+1}-\zeta_{y}\right|$, from which we deduce:

$$
\begin{aligned}
\left\|x_{k+1}-\zeta_{x}\right\| & \leq\left\|x_{k+1}-\phi\left(y_{k+1}\right)\right\|+\left\|\phi\left(y_{k+1}\right)-\zeta_{x}\right\| \\
& \leq\left(\frac{4 \bar{\gamma}_{x}}{\psi\left(u_{x}\right)} L_{\phi}^{2}+l_{\phi} L_{y}\right)\left\|\left(x_{k}, y_{k}\right)-\zeta\right\|^{2} .
\end{aligned}
$$

Combining (48) and (49) gives us:

$$
\left\|\left(x_{k+1}, y_{k+1}\right)-\zeta\right\| \leq\left\|x_{k+1}-\zeta_{x}\right\|+\left|y_{k+1}-\zeta_{y}\right| \leq L_{x}\left\|\left(x_{k}, y_{k}\right)-\zeta\right\|^{2},
$$

and using $\left(A_{19}\right)$ concludes the proof of this part.

5.8. Proof of Part (b) of Theorem 5.1. We let $k:=K$, from Corollary 5.11 and Lemma 5.7 (applied with $(c, b)=\left(z_{k}^{\prime}, y_{k+1}\right)$ ):

$$
\begin{aligned}
\left|y_{k+1}-\zeta_{y}\right| \leq 3 & \frac{m-l^{\prime}}{m}\left(\left(1+(\bar{\mu} \bar{e})^{m}\right) \beta_{m, l^{\prime}}\left(h\left(z_{k}^{\prime}, .\right) ; y_{k+1}\right)\right. \\
& \left.+L_{\bar{e}}^{m /\left(m-l^{\prime}\right)}\left\|z_{k}^{\prime}\right\|^{1 /\left(m-l^{\prime}\right)}\right) .
\end{aligned}
$$

Then, using Part (a) of Lemma 5.13 and the fact that $\mathcal{F}_{m, l, l^{\prime}}^{z}\left(x_{K}, y_{K}\right)=0$, we deduce inequality (30) of the theorem.

From Corollary 5.12, we know $\zeta_{k} \in \bar{B}\left(\zeta_{y}, r_{y}\right)$ and therefore, Lemma 5.7 (applied with $\left.(c, b)=\left(z_{k}^{\prime}, \zeta_{k}\right)\right)$ gives:

$$
\beta_{m, l^{\prime}}\left(h\left(z_{k}^{\prime}, .\right) ; \zeta_{k}\right) \leq \frac{\beta_{m, l^{\prime}}\left(h(0, .) ; \zeta_{k}\right)+L_{\bar{e}}^{m /\left(m-l^{\prime}\right)}\left\|z_{k}^{\prime}\right\|^{1 /\left(m-l^{\prime}\right)}}{1-(\bar{\mu} \bar{e})^{m}} .
$$

Using again $\mathcal{F}_{m, l, l^{\prime}}^{z}\left(x_{K}, y_{K}\right)=0$, we deduce:

$$
\beta_{m, l^{\prime}}\left(h\left(z_{k}^{\prime}, .\right) ; \zeta_{k}\right) \leq \frac{\beta_{m, l^{\prime}}\left(h(0, .) ; \zeta_{k}\right)+L_{\bar{e}}^{m /\left(m-l^{\prime}\right)} \mathcal{B}_{m, l^{\prime}}\left(h\left(z_{k}^{\prime}, .\right), y_{k} ; y_{k+1}\right) / \mathcal{G}_{z}}{1-(\bar{\mu} \bar{e})^{m}} .
$$


In a similar way, using Lemma 5.7 with $(c, b)=\left(z_{k}^{\prime}, \zeta_{y}\right)$, we obtain:

$$
\begin{aligned}
\beta_{m, l^{\prime}}\left(h\left(z_{k}^{\prime}, .\right) ; \zeta_{y}\right) & \leq \frac{\beta_{m, l^{\prime}}+L_{\bar{e}}^{m /\left(m-l^{\prime}\right)}\left\|z_{k}^{\prime}\right\|^{1 /\left(m-l^{\prime}\right)}}{1-(\bar{\mu} \bar{e})^{m}} \\
& \leq \frac{\beta_{m, l^{\prime}}+L_{\bar{e}}^{m /\left(m-l^{\prime}\right)} \mathcal{B}_{m, l^{\prime}}\left(h\left(z_{k}^{\prime}, .\right), y_{k} ; y_{k+1}\right) / \mathcal{G}_{z}}{1-(\bar{\mu} \bar{e})^{m}} .
\end{aligned}
$$

On the other hand, by Corollary 5.12 and $\left(A_{12}\right)$ one has

$$
\gamma_{m}\left(h(0, .) ; \zeta_{k}\right)\left|\zeta_{k}-\zeta_{y}\right| \leq u,
$$

hence Proposition 3.2 yields, via $\left(A_{16}\right)$ :

$$
\beta_{m, l^{\prime}}\left(h(0, .) ; \zeta_{k}\right) \leq \frac{1-u}{\psi_{m}(u)}\left(\beta_{m, l^{\prime}}(1-u)^{\frac{l^{\prime}+1}{m-l^{\prime}}}+(2 m-1)\left|\zeta_{k}-\zeta_{y}\right|\right) .
$$

From Part (a) of Lemma 5.13, we have:

$$
\mathcal{B}_{m, l^{\prime}}\left(h\left(z_{k}^{\prime}, .\right), y_{k} ; y_{k+1}\right) \leq \Xi \beta_{m, l^{\prime}}\left(h\left(z_{k}^{\prime}, .\right) ; \zeta_{k}\right) .
$$

Then, from this inequality and successively using (50), (52), (39) and (51), we deduce:

$$
\mathcal{B}_{m, l^{\prime}}\left(h\left(z_{k}^{\prime}, .\right), y_{k} ; y_{k+1}\right) \leq \Xi\left(T_{1} \beta_{m, l^{\prime}}+T_{2} / \mathcal{G}_{z} \mathcal{B}_{m, l^{\prime}}\left(h\left(z_{k}^{\prime}, .\right), y_{k} ; y_{k+1}\right)\right),
$$

whence (31).

\section{NumericAl EXPERIMENTS}

In this section, we combine results of the two previous sections: from a given starting point we locate clusters, then we compute upper bounds on point estimates at the cluster and enter the approximation algorithm. We relate numerical experiments with the resulting algorithm. Before all, we explain basic devices used in our program.

We consider the following examples parameterized by the real positive number $N$. For the sake of simplicity we restrict to polynomial maps.

Example 1: $f_{1}\left(x_{1}, y\right):=x_{1}+5 x_{1}^{2}, g\left(x_{1}, y\right):=\left(y^{m}-10^{-m N}\right)\left(1-y^{m}\right)$. Here $n=2$ and $(f, g)$ admits a cluster of $m$ zeroes in a neighborhood of the origin, which collapses to the origin when $N$ tends to infinity.

Example 2: $f_{1}\left(x_{1}, x_{2}, y\right):=10^{-4 N}+3 x_{1}+5 x_{2}+y+x_{2} y^{2}+6 x_{1}^{3} x_{2}+5 x_{1} x_{2} y^{2}-$ $5 x_{1} y^{3}-x_{1} y^{4}, f_{2}\left(x_{1}, x_{2}, y\right):=10^{-4 N}-x_{1}+2 x_{2}+x_{1} y-x_{1} x_{2} y-x_{1} y^{3}-4 x_{2} y^{3}+$ $3 x_{1} x_{2}^{3} y, g\left(x_{1}, x_{2}, y\right):=10^{-4 N}+x_{1}+11 / 2 x_{1}^{2}-2 x_{2}+1419 x_{2}^{3}+y^{3}$. Here $n=3$ and $(f, g)$ admits a cluster of 4 zeroes around the origin, which tends to a multiple zero when $N$ goes to infinity.

Computations are performed with the Maple computer algebra system version 7 . The Digits environment variable controls the number of decimal digits that Maple uses when calculating with software long floating-point numbers. Heuristically, in order to avoid rounding off problems, we set this variable to $2 m N$. We leave this precision problem here for the sake of simplicity.

6.1. Approximation of Point Estimates. We describe the formulas we use in our implementation for computing upper bounds on point estimates. 
6.1.1. Computing $\delta_{x}$ and $\sigma_{x}$. At a given point $\left(x_{0}, y_{0}\right)$, we first address the problem of computing $\left\|D \Sigma\left(x_{0}, y_{0}\right)\right\|$ and $\left\|D \Sigma\left(x_{0}, y_{0}\right)^{-1}\right\|$. Both problems correspond to computing norms of matrices $M$ of the form

$$
M=\left(\begin{array}{cc}
\text { Id } & A \\
0 & 1
\end{array}\right),
$$

where $A$ is a column vector of length $n-1$, we use the following classical formula:

Proposition 6.1. [21, Exercise 6.10, p. 116]

$$
\|M\|^{2}=1+\frac{1}{2}\|A\|^{2}+\|A\| \sqrt{1+\frac{1}{4}\|A\|^{2}} .
$$

6.1.2. Majorant Series. Computing majorant series of polynomials reduces to upper bounding the norms of all its derivatives. For this purpose, we make use of the norm $\|\cdot\|_{\infty}$, as defined just below. More sophisticated devices could be used but we retained this one for efficiency and simplicity reasons.

Let $E:=\left\{e_{1}, \ldots, e_{n}\right\}$ denote the canonical basis of $\mathbb{C}^{n}, A \in \mathcal{L}_{l}\left(\mathbb{C}^{n} ; \mathbb{C}^{s}\right)$, it is fast to compute the norm $\|\cdot\|_{\infty}$, defined by

$$
\|A\|_{\infty}:=\max _{u_{1} \in E, \ldots, u_{l} \in E}\left\|A u_{1} \ldots u_{l}\right\| .
$$

Then, we content ourselves with the following upper bound on the norm of $A$ :

Lemma 6.2. $\|A\| \leq n^{l}\|A\|_{\infty}$.

Proof. Let $A_{i_{1}, \ldots, i_{l}}:=A e_{i_{1}} \ldots e_{i_{l}}$. Let $u_{1}, \ldots, u_{l}$ be unit vectors of $\mathbb{C}^{n}$, we compute:

$$
\begin{aligned}
\left\|A u_{1} \ldots u_{l}\right\| & =\left\|\sum_{i_{1}=1}^{n} \cdots \sum_{i_{l}=1}^{n} A_{i_{1}, \ldots, i_{l}} u_{1, i_{1}} \cdots u_{l, i_{l}}\right\| \\
& \leq n^{l}\|A\|_{\infty},
\end{aligned}
$$

where $u_{i, j}$ denotes the $j$ th coordinate of $u_{i}$.

In particular, since the map $\Sigma$ of Theorems 4.1 and 5.1 is polynomial, we use the following upper bound:

$$
\gamma(\Sigma ; a, b) \leq \max _{l \geq 2}\left(n^{l}\left\|D \Sigma(a, b)^{-1} \frac{D^{l} \Sigma(a, b)}{l !}\right\|_{\infty}\right)^{\frac{1}{l-1}} .
$$

Concerning the computations of $\lambda_{g}$ and $\rho_{g}$ at a given point $(a, b)$ such that $[g-g(a, b)]_{(a, b)} \leq \lambda_{g} t /\left(1-\rho_{g} t\right)$, we arbitrarily take:

$$
\lambda_{g}:=\max _{l \geq 1} \frac{n^{l}}{l !}\left\|D^{l} g(a, b)\right\|_{\infty}, \rho_{g}:=1 .
$$




\begin{tabular}{|c|c|c|c|c|c|}
\hline$m$ & 2 & 2 & 2 & 3 & 4 \\
\hline$N$ & 5 & 10 & 20 & 10 & 10 \\
\hline$\left\|\left(x_{0}, y_{0}\right)\right\|$ & $1.3410^{-6}$ & $1.3410^{-6}$ & $1.3410^{-6}$ & $2.6910^{-6}$ & $3.3710^{-7}$ \\
\hline$r_{x}^{-}$ & $6.0910^{-5}$ & $3.6310^{-5}$ & $3.6310^{-5}$ & $2.0810^{-5}$ & $1.4510^{-5}$ \\
\hline$r_{y}^{-}$ & $5.9510^{-5}$ & $3.5210^{-5}$ & $3.5210^{-5}$ & $1.8810^{-5}$ & $1.4310^{-5}$ \\
\hline
\end{tabular}

TABLE 1. Cluster location with Example 1

\begin{tabular}{|c|c|c|c|}
\hline$m$ & 4 & 4 & 4 \\
\hline$N$ & 5 & 10 & 20 \\
\hline$\left\|\left(x_{0}, y_{0}\right)\right\|$ & $1.3210^{-5}$ & $1.3210^{-5}$ & $1.3210^{-5}$ \\
\hline$r_{x}^{-}$ & $1.1510^{-4}$ & $1.1510^{-4}$ & $1.1510^{-4}$ \\
\hline$r_{y}^{-}$ & $9.1510^{-5}$ & $9.1510^{-5}$ & $9.1510^{-5}$ \\
\hline
\end{tabular}

TABle 2. Cluster location with Example 2

6.1.3. Upper Bounds on $\gamma_{m}$. The last basic computation we deal with is the computation of upper bounds on $\gamma_{m}$. The following proposition quantifies how such a bound can be determined from a geometric series majoration, exploiting the possible knowledge of a series expansion.

Proposition 6.3. Let $q$ denote a one complex variable function, $m$ an integer such that $q^{(m)}(z) \neq 0, \sigma_{m} \geq m ! /\left|q^{(m)}(z)\right|$ and $\lambda, \rho$ be nonnegative real numbers such that $[q-q(z)]_{z} \leq \lambda t /(1-\rho t)$. Let $i \geq m+1$, let $p$ denote the unique polynomial of degree at most $i-1$ such that $q-p \in \mathcal{O}_{z}\left((x-z)^{i}\right)$ then

$$
\gamma_{m}(p ; z) \leq \gamma_{m}(q ; z) \leq \max \left(\gamma_{m}(p ; z), \rho\left(\sigma_{m} \lambda \rho^{m-1}\right)^{\frac{1}{i-m}}\right) .
$$

Proof. By construction, we have $\sigma_{m} \lambda \rho^{m-1} \geq 1$, hence

$$
\sup _{j \geq i}\left(\sigma_{m} \lambda \rho^{j-1}\right)^{\frac{1}{j-m}}=\sup _{j \geq i} \rho\left(\sigma_{m} \lambda \rho^{m-1}\right)^{\frac{1}{j-m}}=\rho\left(\sigma_{m} \lambda \rho^{m-1}\right)^{\frac{1}{i-m}} .
$$

6.2. Cluster Location. In Tables 1 and 2, we relate numerical experiments with Theorem 4.1: the parameter $l$ is set to 0 and $\left(x_{0}, y_{0}\right)$ is computed as $(\exp (\imath \pi / 4), \ldots, \exp (\imath \pi / 4))$ times the largest negative power of 2 that satisfies the conditions of the theorem. We indicate the values $r_{x}^{-}$and $r_{y}^{-}$(as defined in the theorem): recall that $\bar{B}\left(x_{0}, r_{x}^{-}\right) \times \bar{B}\left(y_{0}, r_{y}^{-}\right)$contains a cluster of $m$ zeroes.

We observe that the location process does not depend much on the size of the cluster. As expected, the location becomes the more especially difficult as the cardinality of the cluster increases.

We compute the requested upper bound on $\gamma_{m}$ using Proposition 6.3 at $\left(z_{0}^{\prime}, y_{0}\right)$ with $i:=2 m$ and using the geometric series majoration given in (28). The series expansion of $h\left(z_{0}^{\prime},.\right)$ at $y_{0}$ is directly computed from the one of $\Phi\left(z_{0}^{\prime},.\right)$, which is obtained by means of the classical symbolic Newton iteration. 
This location process provides us a ball $\bar{B}\left(\left(x_{0}, y_{0}\right), r\right)$ that contains the cluster, when taking $r:=\sqrt{\left(r_{x}^{-}\right)^{2}+\left(r_{y}^{-}\right)^{2}}$. We next detail how one can make use of Theorem 5.1 from this radius $r$ and the only estimates at the initial point $\left(x_{0}, y_{0}\right)$.

6.3. Algorithm from Estimates at the Initial Point. We are now ready to describe how valid input quantities of Theorem 5.1 can be computed from estimates at the initial point only. The strategy is the same as in Section 1.4: we perform cluster location first and then deduce upper bounds on point estimates in the cluster.

The initial point is still written $\left(x_{0}, y_{0}\right)$. We assume that the location criterion underlying Theorem 4.1 holds. Namely, we assume that there exists a cluster of $m-l^{\prime}$ zeroes of $\left(f, g^{\left[l^{\prime}\right]}\right)$ in $\bar{B}\left(\left(x_{0}, y_{0}\right), r\right)$. From these data, we attempt to apply Theorem 5.1, as explained in the following algorithm. Of course, the process breaks as soon as a computation is not possible or a requirement fails. From now, $\zeta$ represents a point of the cluster.

- At $\left(x_{0}, y_{0}\right)$, compute upper bounds $\gamma_{x_{0}}, \delta_{x_{0}}$ and $\sigma_{x_{0}}$ on $\gamma\left(\Sigma ; x_{0}, y_{0}\right)$, $\left\|D \Sigma\left(x_{0}, y_{0}\right)\right\|$ and $\left\|D \Sigma\left(x_{0}, y_{0}\right)^{-1}\right\|$ respectively. Use Proposition 1.15 to compute (if possible) upper bounds $\gamma_{x}, \delta_{x}, \sigma_{x}$ of $\gamma(\Sigma ; \zeta),\|D \Sigma(\zeta)\|$ and $\left\|D \Sigma(\zeta)^{-1}\right\|$, respectively (hence $\left(A_{1}\right)$ is satisfied).

- At $\left(x_{0}, y_{0}\right)$ compute suitable values for $\lambda_{g, x_{0}}$ and $\rho_{g, x_{0}}$ in order to have $\left[g-g\left(x_{0}, y_{0}\right)\right]_{\left(x_{0}, y_{0}\right)} \leq \lambda_{g, x_{0}} t /\left(1-\rho_{g, x_{0}} t\right)$. Use Corollary 1.6 to compute (if possible) $\lambda$ and $\rho$ satisfying $\left(A_{2}\right)$.

- Compute $\lambda_{\Phi}, \rho_{\Phi}, \lambda, \rho$, require $\left(A_{3}\right)$ and compute $l_{\phi}, L_{\phi}$ and $r_{x}$.

- Use Proposition 1.15 to compute (if possible) upper bounds $\bar{\gamma}_{x}$ and $\bar{\delta}_{x}$ of $\max \left(\gamma(\Sigma ; x, y) \mid(x, y) \in \bar{B}\left(\zeta, l_{\phi} r\right)\right)$ and $\max (\|D \Sigma(x, y)\| \mid(x, y) \in$ $\left.\bar{B}\left(\zeta, l_{\phi} r\right)\right)$. According to Lemma 5.3, conditions $\left(A_{4}\right)$ and $\left(A_{5}\right)$ are satisfied. Invoking this lemma is legitimate since it only makes use of $\left(A_{1}\right)$, $\left(A_{2}\right)$ and $\left(A_{3}\right)$. This is the reason why we have introduced $\left(A_{3}\right)$ in the statement of Theorem 5.1 although it is a consequence of $\left(A_{9}\right)$.

- Compute $u_{x}$, require $\left(A_{6}\right)$ and compute $L_{z}, r_{z}$.

- Compute $x_{0}^{\prime}, z_{0}^{\prime}$.

- Compute the series expansion of $h\left(z_{0}^{\prime},.\right)$ at $y_{0}$ at precision $2\left(m-l^{\prime}\right)$ and, then, upper bounds on $\beta_{m, l^{\prime}}\left(h\left(z_{0}^{\prime},.\right) ; y_{0}\right), m ! /\left|D_{y}^{m} h\left(z_{0}^{\prime}, y_{0}\right)\right|$. From $\lambda, \rho$ and using Corollary 1.6, compute (if possible) a geometric majorant series $\lambda^{\prime} t /\left(1-\rho^{\prime} t\right)$ of $h-h\left(z_{0}^{\prime}, y_{0}\right)$ at $\left(z_{0}^{\prime}, y_{0}\right)$. Then use Proposition 6.3 to compute an upper bound on $\gamma_{m}\left(h\left(z_{0}^{\prime},.\right) ; y_{0}\right)$ (we set the parameter $i$ to $2\left(m-l^{\prime}\right)$ in our program $)$.

- Use $\lambda^{\prime}, \rho^{\prime}$ and Proposition 3.6 to compute (if possible) upper bounds on:

$$
\begin{aligned}
& \max \left(\beta_{m, l^{\prime}}\left(h(z, .) ; y_{0}\right) \mid z \in \bar{B}\left(z_{0}^{\prime},\left\|z_{0}^{\prime}\right\|+r_{z}\right)\right), \\
& \max \left(\gamma_{m}\left(h(z, .) ; y_{0}\right) \mid z \in \bar{B}\left(z_{0}^{\prime},\left\|z_{0}^{\prime}\right\|+r_{z}\right)\right), \\
& \max \left(m ! /\left|D_{y}^{m} h\left(z, y_{0}\right)\right| \mid z \in \bar{B}\left(z_{0}^{\prime},\left\|z_{0}^{\prime}\right\|+r_{z}\right)\right) .
\end{aligned}
$$

- Use Proposition 3.2 and the previous quantities to compute (if possible) an upper bound on

$$
3 \frac{m-l^{\prime}}{m} \max \left(\beta_{m, l^{\prime}}(h(z, .) ; y) \mid z \in \bar{B}\left(z_{0}^{\prime},\left\|z_{0}^{\prime}\right\|+r_{z}\right), y \in \bar{B}\left(y_{0}, 2 r\right)\right)
$$




\begin{tabular}{|c|c|c|c|c|}
\hline$N$ & 10 & 20 & 40 & 80 \\
\hline$\left\|\left(x_{0}, y_{0}\right)\right\|$ & $2.0510^{-11}$ & $2.0510^{-11}$ & $2.0510^{-11}$ & $2.0510^{-11}$ \\
\hline$r_{y}$ & $1.9610^{-8}$ & $1.3910^{-8}$ & $1.3910^{-8}$ & $1.3910^{-8}$ \\
\hline $\mathcal{G}_{y}$ & $1.0510^{5}$ & $1.0510^{5}$ & $1.0510^{5}$ & $1.0510^{5}$ \\
\hline $\mathcal{G}_{z}$ & $6.8010^{3}$ & $6.7910^{3}$ & $6.7910^{3}$ & $6.7910^{3}$ \\
\hline$K$ & 0 & 1 & 2 & 3 \\
\hline $\mathcal{F}_{m, l, l^{\prime}}^{y}$ & - & $1,-$ & $1,+,-$ & $1,1,-,-$ \\
\hline $\mathcal{F}_{m, l, l^{\prime}}^{z}$ & 0 & 1,0 & $1,1,0$ & $1,1,1,0$ \\
\hline$\left\|x_{K+1}\right\|$ & $1.0510^{-21}$ & $5.6010^{-42}$ & $1.5710^{-82}$ & $1.2310^{-163}$ \\
\hline$\left|y_{K+1}\right|$ & $1.4510^{-11}$ & $6.8710^{-30}$ & $3.2410^{-48}$ & $2.9210^{-98}$ \\
\hline $\mathcal{B}_{m, l^{\prime}}\left(y_{K+1}\right)$ & $1.0010^{-10}$ & $1.0010^{-20}$ & $1.0010^{-40}$ & $1.0010^{-80}$ \\
\hline
\end{tabular}

TABLE 3. Cluster approximation with Example 1 and $m=2$

\begin{tabular}{|c|c|c|c|c|}
\hline$N$ & 10 & 20 & 40 & 80 \\
\hline$\left\|\left(x_{0}, y_{0}\right)\right\|$ & $1.0210^{-11}$ & $1.0210^{-11}$ & $1.0210^{-11}$ & $1.0210^{-11}$ \\
\hline$r_{y}$ & $3.9010^{-8}$ & $2.6210^{-8}$ & $2.6210^{-8}$ & $2.6210^{-8}$ \\
\hline $\mathcal{G}_{y}$ & $1.2010^{6}$ & $1.1910^{6}$ & $1.1910^{6}$ & $1.1910^{6}$ \\
\hline $\mathcal{G}_{z}$ & $9.5910^{4}$ & $9.4910^{4}$ & $9.4910^{4}$ & $9.4910^{4}$ \\
\hline$K$ & 0 & 1 & 2 & 3 \\
\hline $\mathcal{F}_{m, l, l^{\prime}}^{y}$ & $\infty$ & $1, \infty$ & $1, \infty, \infty$ & $1,+, \infty, \infty$ \\
\hline $\mathcal{F}_{m, l, l^{\prime}}^{z}$ & 0 & 1,0 & $1,1,0$ & $1,1,1,0$ \\
\hline$\left\|x_{K+1}\right\|$ & $2.6410^{-22}$ & $3.5010^{-43}$ & $6.1310^{-85}$ & $1.8810^{-168}$ \\
\hline$\left|y_{K+1}\right|$ & $7.2710^{-12}$ & $2.5910^{-47}$ & $2.0310^{-56}$ & $1.1710^{-153}$ \\
\hline $\mathcal{B}_{m, l^{\prime}}\left(y_{K+1}\right)$ & $1.0010^{-10}$ & $9.9910^{-21}$ & $1.0010^{-40}$ & $1.0010^{-80}$ \\
\hline
\end{tabular}

TABLE 4. Cluster approximation with Example 1 and $m=4$

and take $r_{y}$ as the maximum of $r$ and the last upper bound, so that conditions $\left(A_{7}\right)$ and $\left(A_{8}\right)$ are satisfied.

- Compute $\bar{r}$ and require $\left(A_{9}\right)$.

- Use Proposition 3.2 to compute valid values for $\bar{\beta}_{m}$ and $\bar{\gamma}_{m}$ and $\bar{\sigma}_{m}$, in order to satisfy $\left(A_{10}\right),\left(A_{11}\right)$ and $\left(A_{12}\right)$.

- Compute $\bar{\lambda}, \bar{\rho}, \bar{\mu}, L_{\bar{e}}, \bar{e}$ and require $\left(A_{13}\right)$.

- Require $\left(A_{14}\right)$ and $\left(A_{15}\right)$.

- Compute $u, v, \bar{u}$ and require $\left(A_{16}\right)$.

- Compute $\mathcal{C}, \mathcal{G}_{y}, \overline{\mathcal{C}}, \underline{\kappa}, \bar{\kappa}, \chi, \Xi, T_{1}, T_{2}, T_{\beta}, \mathcal{G}_{z}, T_{y}$ and require $\left(A_{17}\right)$.

- Compute $L_{y, 1}, L_{y, 2}, L_{y}$ and require $\left(A_{18}\right)$.

- Compute $L_{x}$ and require $\left(A_{19}\right)$.

It is straighforward to check that if $\zeta$ is an isolated zero of multiplicty $m$ then, for any $l \in\{0, \ldots, m-1\}, l^{\prime} \leq l$, this algorithm works with $\left(x_{0}, y_{0}\right):=\zeta$ 


\begin{tabular}{|c|c|c|c|c|}
\hline$N$ & 10 & 20 & 40 & 80 \\
\hline$\left\|\left(x_{0}, y_{0}\right)\right\|$ & $2.5210^{-11}$ & $2.5210^{-11}$ & $2.5210^{-11}$ & $2.5210^{-11}$ \\
\hline$r_{y}$ & $1.9110^{-8}$ & $1.1910^{-8}$ & $1.1910^{-8}$ & $1.1910^{-8}$ \\
\hline $\mathcal{G}_{y}$ & $2.0410^{5}$ & $2.0410^{5}$ & $2.0410^{5}$ & $2.0410^{5}$ \\
\hline $\mathcal{G}_{z}$ & $2.6910^{5}$ & $2.6910^{5}$ & $2.6910^{5}$ & $2.6910^{5}$ \\
\hline$K$ & 0 & 1 & 1 & 2 \\
\hline $\mathcal{F}_{m, l, l^{\prime}}^{y}$ & - & $1,-$ & $1,+$ & $1,1,+$ \\
\hline $\mathcal{F}_{m, l, l^{\prime}}^{z}$ & 0 & 0,0 & 0,0 & $0,0,0$ \\
\hline$\left\|x_{K+1}\right\|$ & $2.9510^{-12}$ & $7.4610^{-25}$ & $4.7610^{-50}$ & $1.9310^{-100}$ \\
\hline$\left|y_{K+1}\right|$ & $1.4510^{-11}$ & $3.6710^{-24}$ & $2.3410^{-49}$ & $9.5210^{-100}$ \\
\hline $\mathcal{B}_{m, l^{\prime}}\left(y_{K+1}\right)$ & $9.5910^{-11}$ & $9.5910^{-21}$ & $9.5910^{-41}$ & $9.5910^{-81}$ \\
\hline
\end{tabular}

TABle 5. Cluster approximation with Example 2

and $r=0$ (in particular this implies $\bar{r}=r_{x}=r_{y}=r_{z}=0$ ). By continuity, we deduce that if $\left(x_{0}, y_{0}\right)$ is sufficiently close to $\zeta$ and $r$ sufficiently small then the algorithm also works. By deformation, it follows that the algorithm actually locates and approximates clusters of embbeding dimension 1 .

For clusters with positive diameter, the iteration stops. At the end, Theorem 5.1 asserts that $y_{K+1}$ is close to $\zeta_{y}$ at a distance bounded in terms of $\beta_{m, l^{\prime}}\left(h(0,.) ; \zeta_{y}\right)$. According to [12, Theorem 2.1], this quantity can be bounded in terms of the diameter of the cluster of zeroes of $h(0,$.$) (if the$ diameter of the cluster is sufficiently small). As for the $x$ coordinates, no such result actually holds. Nevertheless, one can iterate the Newton operator $x \mapsto N\left(f\left(., y_{K+1}\right) ; x\right)$ from $x_{K+1}$ to improve the $x$ coordinates. The convergence of this iteration can be quantified by means of Lemma 5.4 and the iteration can be stopped as soon as it reaches a distance to $\phi\left(y_{K+1}\right)$ which is about $\mathcal{B}_{m, l^{\prime}}\left(h\left(z_{K}^{\prime},.\right), y_{K} ; y_{K+1}\right)$. We leave out details here.

In Tables 3, 4 and 5, we report on numerical experiments: we take $l^{\prime}:=l:=0$ and, for different values of $m$ and $N$, we compute $\left(x_{0}, y_{0}\right)$ as the vector $(\exp (\imath \pi / 4), \ldots, \exp (\imath \pi / 4))$ times the largest negative power of 2 that does not provoke an error in the whole algorithm. We indicate the values of the main parameters, namely $r_{y}, \mathcal{G}_{y}$ and $\mathcal{G}_{z}$. Then we give the number $K$ of iterations, the sequences of flags $\left(\mathcal{F}_{m, l, l^{\prime}}^{y}\left(x_{k}, y_{k}\right)\right)_{k}$ and $\left(\mathcal{F}_{m, l, l^{\prime}}^{z}\left(x_{k}, y_{k}\right)\right)_{k}$, the norms of the last iterate and the value of $\mathcal{B}_{m, l^{\prime}}\left(h\left(z_{K}^{\prime},.\right), y_{K} ; y_{K+1}\right)$, abbreviated $\mathcal{B}_{m, l^{\prime}}\left(y_{K+1}\right)$.

In all our examples the diameter of the cluster is about $10^{-N}$, as confirmed by the values of $\mathcal{B}_{m, l^{\prime}}\left(h\left(z_{K}^{\prime},.\right), y_{K} ; y_{K+1}\right)$. In all cases, the $x$ coordinates are already close to the cluster without performing the aforementioned posttreatment. Lastly, it is important to observe that all the eight possible cases of the algorithm actually occur in practice. 


\section{REFERENCES}

[1] AĬZenberg, I. A., And Yuzhakov, A. P. Integral representations and residues in multidimensional complex analysis, vol. 58 of Translations of Mathematical Monographs. American Mathematical Society, Providence, RI, 1983. Translated from the Russian by H. H. McFaden, Translation edited by Lev J. Leifman.

[2] Allgower, E. L., Böhmer, K., Hoy, A., And Janovský, V. Direct methods for solving singular nonlinear equations. ZAMM Z. Angew. Math. Mech. 79, 4 (1999), 219-231.

[3] Arnol'd, V. I., GuseǏn-Zade, S. M., And Varchenko, A. N. Singularities of differentiable maps. Vol. I, vol. 82 of Monographs in Mathematics. Birkhäuser Boston Inc., Boston, MA, 1985. The classification of critical points, caustics and wave fronts, Translated from the Russian by Ian Porteous and Mark Reynolds.

[4] Blum, L., Cucker, F., Shub, M., And Smale, S. Complexity and real computation. Springer-Verlag, New York, 1998. With a foreword by Richard M. Karp.

[5] Bondyfalat, D., Mourrain, B., And Pan, V. Y. Computation of a specified root of a polynomial system of equations using eigenvectors. Linear Algebra Appl. 319, 1-3 (2000), 193-209.

[6] Decker, D. W., ANd Kelley, C. T. Newton's method at singular points. I. SIAM J. Numer. Anal. 17, 1 (1980), 66-70.

[7] Decker, D. W., ANd Kelley, C. T. Newton's method at singular points. II. SIAM J. Numer. Anal. 17, 3 (1980), 465-471.

[8] Dedieu, J.-P., Kim, M.-H., Shub, M., And Tisseur, F. Implicit gamma theorems. I. Pseudoroots and pseudospectra. Found. Comput. Math. 3, 1 (2003), 1-31.

[9] Dedieu, J.-P., And Shub, M. On simple double zeros and badly conditioned zeros of analytic functions of $\mathrm{n}$ variables. Math. Comp. (2001), 319-327.

[10] FAugÈre, J.-C. A new efficient algorithm for computing Gröbner bases $\left(F_{4}\right)$. $J$. Pure Appl. Algebra 139, 1-3 (1999), 61-88. Effective methods in algebraic geometry (Saint-Malo, 1998).

[11] FAugère, J.-C. A new efficient algorithm for computing Gröbner bases without reduction to zero (F5). In Proceedings of the 2002 International Symposium on Symbolic and Algebraic Computation (2002), T. Mora, Ed., ACM Press, pp. 75-83.

[12] Giusti, M., Lecerf, G., Salvy, B., and Yakoubsohn, J.-C. On location and approximation of clusters of zeroes of analytic functions. Manuscript, 2004.

[13] Govaerts, W. Computation of singularities in large nonlinear systems. SIAM J. Numer. Anal. 34, 3 (1997), 867-880.

[14] Griewank, A. Starlike domains of convergence for Newton's method at singularities. Numer. Math. 35, 1 (1980), 95-111.

[15] Griewank, A. On solving nonlinear equations with simple singularities or nearly singular solutions. SIAM Rev. 27, 4 (1985), 537-563.

[16] Griewank, A., And Osborne, M. R. Analysis of Newton's method at irregular singularities. SIAM J. Numer. Anal. 20, 4 (1983), 747-773.

[17] Griewank, A., and Reddien, G. W. Characterization and computation of generalized turning points. SIAM J. Numer. Anal. 21, 1 (1984), 176-185.

[18] Griewank, A., And Reddien, G. W. The approximate solution of defining equations for generalized turning points. SIAM J. Numer. Anal. 33, 5 (1996), 1912-1920.

[19] Henrici, P. Applied and computational complex analysis. Wiley Classics Library. John Wiley \& Sons Inc., 1991.

[20] Hermann, M., Kunkel, P., and Middelmann, W. Augmented systems for the computation of singular points in Banach space problems. ZAMM Z. Angew. Math. Mech. 78, 1 (1998), 39-50.

[21] Higham, N. J. Accuracy and stability of numerical algorithms, second ed. Society for Industrial and Applied Mathematics (SIAM), Philadelphia, PA, 2002.

[22] Hoy, A. A relation between Newton and Gauss-Newton steps for singular nonlinear equations. Computing 40, 1 (1988), 19-27. 
[23] Hoy, A. Analysis of a bordering approach for solving singular nonlinear equations. Wiss. Z. Martin-Luther-Univ. Halle-Wittenberg Math.-Natur. Reihe 38, 4 (1989), $115-121$.

[24] Kantorovich, L. V., And Akilov, G. P. Functional analysis, second ed. Pergamon Press, 1982. Translated from the Russian by Howard L. Silcock.

[25] Kearfott, R. B., Dian, J., and Neumaier., A. Existence verification for singular zeros of complex nonlinear systems. SIAM J. Numer. Anal. 38, 2 (2000), 360-379.

[26] Kravanja, P., And Hazgemans, A. A modification of Newton's method for analytic mappings having multiple zeros. Computing 62, 2 (1999), 129-145.

[27] Kravanja, P., And VAn Barel, M. Computing the zeros of analytic functions, vol. 1727 of Lecture Notes in Mathematics. Springer-Verlag, Berlin, 2000.

[28] Kunkel, P. Quadratically convergent methods for the computation of unfolded singularities. SIAM J. Numer. Anal. 25, 6 (1988), 1392-1408.

[29] Kunkel, P. Efficient computation of singular points. IMA J. Numer. Anal. 9, 3 (1989), 421-433.

[30] Kunkel, P. A tree-based analysis of a family of augmented systems for the computation of singular points. IMA J. Numer. Anal. 16, 4 (1996), 501-527.

[31] LeCERF, G. Quadratic Newton iteration for systems with multiplicity. Found. Comput. Math. 2, 3 (2002), 247-293.

[32] Leykin, A., Verschelde, J., And Zhao, A. Newton's method with deflation for isolated singularities of polynomial systems. Manuscript, 2004.

[33] MEI, Z. A special extended system and a Newton-like method for simple singular nonlinear equations. Computing 45, 2 (1990), 157-167.

[34] Morgan, A. P., Sommese, A. J., And Wampler, C. W. Computing singular solutions to polynomial systems. Adv. in Appl. Math. 13, 3 (1992), 305-327.

[35] OJIKA, T. Modified deflation algorithm for the solution of singular problems. I. a system of nonlinear algebraic equations. J. Math. Anal. Appl. 123 (1987), 199-221.

[36] Ojika, T., Watanabe, S., and Mitsui, T. Deflation algorithm for the multiple roots of a system of nonlinear equations. J. Math. Anal. Appl. 96, 2 (1983), 463-479.

[37] Ostrowski, A. M. Solution of equations and systems of equations. Academic Press, New York, NY, 1966.

[38] Pellet, A.-E. Sur un mode de séparation des racines des équations et la formule de Lagrange. Bulletin des Sciences Mathématiques et Astronomiques 5 (1881), 393-395. Deuxième Série, Tome 16 de la Collection.

[39] Rabier, P. J., And Reddien, G. W. Characterization and computation of singular points with maximum rank deficiency. SIAM J. Numer. Anal. 23, 5 (1986), 10401051.

[40] RALL, L. Convergence of the Newton process to multiple solutions. Num. Math. 9 (1966), 23-37.

[41] Reddien, G. W. On Newton's method for singular problems. SIAM J. Numer. Anal. 15, 5 (1978), 993-996.

[42] Reddien, G. W. Newton's method and high order singularities. Comput. Math. Appl. 5, 2 (1979), 79-86.

[43] SchröDER, E. Über unendlich viele Algorithmen zur Auflösung der Gleichungen. Math. Annalen 2 (1870), 317-365.

[44] Shub, M., And Smale, S. Computational complexity. On the geometry of polynomials and a theory of cost. I. Ann. Sci. École Norm. Sup. (4) 18, 1 (1985), 107-142.

[45] Shub, M., And Smale, S. Computational complexity: on the geometry of polynomials and a theory of cost. II. SIAM J. Comput. 15, 1 (1986), 145-161.

[46] Smale, S. The fundamental theorem of algebra and complexity theory. Bull. Amer. Math. Soc. (N.S.) 4, 1 (1981), 1-36.

[47] Smale, S. Newton method estimates from data at one point. In In The Merging of Disciplines: New Directions in Pure, Applied, and Computational Mathematics, R. E. Ewing, K. I. Gross, and C. F. Martin, Eds. Springer-Verlag, 1986, pp. 185-196.

[48] Sommese, A. J., And Verschelde, J. Numerical homotopies to compute generic points on positive dimensional algebraic sets. J. Complexity 16, 3 (2000), 572-602. 
[49] Sommese, A. J., Verschelde, J., And Wampler, C. W. A method for tracking singular paths with application to the numerical irreducible decomposition. In Algebraic geometry. de Gruyter, Berlin, 2002, pp. 329-345.

[50] Sommese, A. J., And WAmpler, C. W. Numerical algebraic geometry. In The mathematics of numerical analysis (Park City, UT, 1995), vol. 32 of Lectures in Appl. Math. Amer. Math. Soc., Providence, RI, 1996, pp. 749-763.

[51] Stenger, F. Computing the topological degree in $\mathbb{R}^{n}$. Numer. Math. 25 (1975), 23-38.

[52] TsuchiyA, T. Enlargement procedure for resolution of singularities at simple singular solutions of nonlinear equations. Numer. Math. 52, 4 (1988), 401-411.

[53] VAN DE VEL, H. A method for computing a root of a single nonlinear equation, including its multiplicity. Computing 14, 1-2 (1975), 167-171.

[54] Vander Straeten, M., And Van de Vel, H. Multiple root-finding methods. J. Comput. Appl. Math. 40, 1 (1992), 105-114.

[55] WANG, X. H., AND HAN, D. F. On dominating sequence method in the point estimate and Smale theorem. Sci. China, Series A 33, 2 (1990), 135-144.

[56] Weber, H., AND Werner, W. On the accurate determination of nonisolated solutions of nonlinear equations. Computing 26, 4 (1981), 315-326.

[57] Yамамото, N. Regularization of solutions of nonlinear equations with singular Jacobian matrices. J. Inform. Process. 7, 1 (1984), 16-21.

Marc Giusti, Laboratoire STIX, École polytechnique, 91128 Palaiseau, France E-mail address: Marc.Giusti@polytechnique.fr

Grégoire Lecerf, Laboratoire de Mathématiques, Université de Versailles Saint-Quentin-en-Yvelines, 45 avenue des États-Unis, 78035 Versailles, France E-mail address: Gregoire.Lecerf@math.uvsq.fr

Bruno Salvy, Projet Algo, inRIA Rocquencourt, 78153 Le Chesnay, France E-mail address: Bruno.Salvy@inria.fr

Jean-Claude Yakoubsohn, Laboratoire MiP, Bureau 131, Université Paul Sabatier, 118 route de Narbonne, 31062 Toulouse, France

E-mail address: yak@mip.ups-tlse.fr 